\section{PROGRAMA DE DOCTORADO EN INGENIERÍA INDUSTRIAL}

\title{
COORDINATING INDUSTRIAL PRODUCTION AND COGENERATION SYSTEMS TO EXPLOIT ELECTRICITY PRICE FLUCTUATIONS
}

Presentada por Cristian Pablos de la Fuente para optar al grado de

Doctor/a por la Universidad de Valladolid

\author{
Dirigida por: \\ Dr. Luis Felipe Acebes Arconada \\ Dr. Alejandro Merino Gómez
}



This work was supported by the Spanish Government under research projects DPI2015-70975-P and PGC2018-099312-B-C31, and by the regional government of Castilla y León plus the EU-FEDER (CLU 2017-09 and UIC 233).

The author was also recipient of an FPI grant (BES-2016-079388) from the Spanish Science and Innovation Ministry (MICINN). 

"Es de importancia para quien desea alcanzar una certeza en su investigación, el saber dudar a tiempo." Aristóteles 



\section{Agradecimientos}

En primer lugar, me gustaría dar las gracias a mis directores, L. Felipe Acebes y Alejandro Merino, por su guía a lo largo de esta tesis, por todo el tiempo que me han dedicado, y por haberme dado ánimos y confianza cuando parecía que llegábamos a un callejón sin salida. Además, me gustaría agradecer a César de Prada la oportunidad que me dio para poder llevar a cabo esta tesis y todos los sabios consejos que me ha dado a lo largo de su ejecución. En este punto también me gustaría dar las gracias a José Luis Pitarch, por todas las arduas discusiones que hemos mantenido sobre mi tesis a lo largo de estos años y que han servido para perfilar el resultado final, y por todo el tiempo que me ha dedicado sin ningún motivo más que el de querer ayudarme.

En segundo lugar, doy las gracias por su aceptación, ayuda, y buenos momentos a todos los miembros del departamento de Ingeniería de Sistemas y Automática de la Universidad de Valladolid, y especialmente a los pertenecientes al grupo de Control y Supervisión de Procesos. Por supuesto, dentro del mismo, no puedo olvidarme de todos mis compañeros de la sala de investigadores del Alfonso: José, Carlos, Pedro, Aníbal, Erika, Fernando, Hicham, Irina, Álvaro y Tania, sin los cuales estoy convencido de que el desarrollo de esta tesis hubiera sido un trabajo mucho más duro y aburrido. Espero que a todos os vaya muy bien en la vida y podáis alcanzar todas vuestras metas en el futuro. Una especial mención para Pablo y María quienes además de compañeros se han convertido en grandes amigos y me han ayudado a superar momentos personales muy difíciles a lo largo del desarrollo de esta tesis. 
En tercer lugar, quiero agradecer al Prof. Lorenz T. Biegler, a todos los miembros de su grupo de investigación, y al Center for Advanced Process Decision-Making (CAPD) en Carnegie Mellon University (CMU), por su recibimiento y por toda la ayuda que me brindaron durante los meses que pasé en Estados Unidos. Gracias a ellos pude reorientar la tesis en un momento crítico y encaminarla hacia lo que ha sido finalmente.

Claramente, en este apartado no podrían faltar todos mis amigos, los cuáles me han ayudado a desconectar cuando lo necesitaba y a mostrarme que había más vida fuera del doctorado.

Doy gracias también a mi familia y en especial a mis padres, los cuales siempre me han apoyado y me han enseñado que, con esfuerzo, motivación, y una sonrisa, siempre se puede seguir adelante y salir de cualquier situación por dura y difícil que sea.

Y, por último, y no menos importante, a mi novia y compañera de vida Suní. Gracias por todos los buenos momentos que hemos pasado y los que nos quedan por vivir, por toda la paciencia que has tenido conmigo durante estos años, y en definitiva por haber estado conmigo a las duras y a las maduras. Por todo ello, y por mucho más, siempre tendrás un lugar muy especial en mi corazón. 


\section{Abstract}

Electricity price fluctuations, given by the application of price-based demand response programs, provide a big opportunity to reduce the operating costs of industries working with on-site cogeneration systems. Thus, they can adapt their production to generate and consume energy in the most appropriate moment, while trading the electricity surplus/deficit with the external grid.

Currently, due to the sugar market liberalization, the beet-sugar extraction industry is at a delicate moment in Europe. This problem, joint to the fact that such industry is a common cogeneration user, makes it an excellent candidate to exploit the aforementioned opportunities. However, the problem lies in the modification of the operating conditions of industrial processes working with simple cogeneration systems, where the electricity generation is determined by the main process heat demand, which in turn depends on its operating conditions. This means that a coupling between the electricity generation and the industrial process operation exists and must be considered. Furthermore, the great complexity of such processes, as is the case in the beet-sugar extraction process, makes that changes in the operating conditions may cause large transition periods between stationary points, which can easily exceed the frequency electricity prices change. In addition, to dispose the electricity with guarantees, a series of efficiency limits, established by the current legislation regarding the efficient use of cogeneration systems, must be respected. These features make the electricity trading forecasting difficult to manage, and often limits the participation of such companies in demand-response programs. 
To solve this problem, decision support tools emerge as a possible solution to facilitate the simultaneous management of production and cogeneration operation, while considering external constraints from sources like the electricity market or the current legislation. However, so far, no methodology has been found in the literature that adequately considers the big inertia present in such industrial processes and its consequences on the electricity trading and, therefore, these methods cannot be applied directly on a process like the one considered. One of the main reasons for not including dynamic features in optimization problems is the higher computational cost they involve. Therefore, in this thesis, the main objective is to develop a new methodology so industrial processes with coupled cogeneration systems can be managed in a simultaneous way, while taking explicit consideration of its dynamic features. Such methodology must be applicable to the beet-sugar industry and extensible to others that share similar characteristics. Thus, we propose an approach based on dynamic optimization techniques, where industrial process and cogeneration system are considered in a simultaneous way (integrated approach), so the effect of the energetic coupling is explicitly considered.

In order to carry out this approach, we need to obtain mathematical models that adequately represent the dynamic features of the system as a whole (Industrial process-cogeneration plant), but without involving an excessive computational load when they are evaluated. Since a nonlinear dynamic optimization is posed, the computational time needed to solve the problem grows exponentially when the complexity and the size of the model increase. Therefore, we propose the use of gray models composed of first-principles equations and empirical relationships, which represent the whole system closedloop dynamics, and can be obtained in a simple way.

To validate the proposed methodology, a dynamic simulator was developed representing a generic beet-sugar factory, and it has been used to test two different electricity tariffs given by price-based demand-response programs: Time-off-use (TOU) tariffs and Day-Ahead market prices. In the study, we have considered the Spanish electricity market, the current legislation related to the efficient operation of cogeneration systems, and the seasonality of the beetsugar extraction process in Spain. To solve the optimization problem, a simultaneous approach based on orthogonal collocation has been used, in which all the equations of the model are discretized and solved using a gradient-based 
algorithm such as IPOPT. Thus, the accuracy of the model has been reduced in the search of a higher computational efficiency.

As main study results, we have obtained that a reduction of up to $2.55 \%$ of the operating costs is possible if a typical TOU program is selected, and a $5.41 \%$ if the electricity market prices are directly used. Of course, this number depends on the period selected, but we can conclude that better results are obtained when the Day-Ahead market price is considered at a cost of a higher uncertainty in the price. In both cases the duration of the campaign has been extended by 3 days compared to a non-energy awareness approach, typically used nowadays in sugar factories, where the production rate has been maintained constantly at the maximum possible level.

Finally, aware of the difficulty that may involve the industrial implementation of a tool like the one developed, we propose a software architecture, with three different modules, communicated using the OPC UA standard: an optimization module developed using the programming language Python ${ }^{\mathrm{TM}}$; a simulator implemented with Ecosimpro/Proosis ${ }^{\circledR}$ modeling and simulation software; and a supervision system developed with Wonderware ${ }^{\circledR}$ Intouch ${ }^{\circledR}$, the latter being the link and core of the whole system. 



\section{Resumen}

Las fluctuaciones en el precio de la electricidad, procedentes de la aplicación de programas de respuesta de la demanda, son una oportunidad para que las industrias que cuenten con sistemas de cogeneración para abastecerse energéticamente puedan minimizar los costes variables de producción del conjunto, produciendo la energía en el momento más adecuado e intercambiando el excedente/déficit de electricidad con la red externa.

La actual crisis en la industria de extracción de azúcar de remolacha en Europa, provocada por la liberalización del mercado azucarero y que ha derivado en el cierre de numerosas empresas de este sector, unido a que dicha industria es usuaria común de la cogeneración, hace que sea un excelente candidato para explotar las mencionadas oportunidades. Sin embargo, la problemática se encuentra en la adaptación de la producción de procesos industriales que cuentan con sistemas de cogeneración simples, donde la generación de electricidad viene marcada por la demanda de calor por parte del proceso principal asociado, que a su vez depende de las condiciones de operación del mismo. Esto hace que exista un acoplamiento entre la generación de electricidad y la operación del proceso industrial. Además, hay que tener en cuenta que debido a la gran complejidad que presentan en muchas ocasiones los procesos industriales, como es el caso de la industria azucarera, cambios en las condiciones de operación provocan largos periodos de transición entre puntos de operación estacionarios, los cuales pueden superar fácilmente la frecuencia con la que los precios de la electricidad cambian. Además, debe tenerse en 
cuenta que para poder verter con garantías la energía eléctrica generada en sistemas de cogeneración, debe respetarse una serie de límites de eficiencia establecidos por la legislación vigente. Todo esto hace que la previsión de compraventa de electricidad sea difícil de gestionar, y en muchas ocasiones limita la participación en programas de respuesta de la demanda por parte de empresas con estas características.

Para solucionar esta problemática, se pueden utilizar herramientas de ayuda a la toma de decisiones, que permitan facilitar la gestión simultánea de la producción del proceso industrial y la operación de su planta de cogeneración asociada, de forma que, además, tengan en cuenta restricciones externas procedentes de fuentes como el mercado eléctrico o la legislación vigente. Sin embargo, revisando la literatura relacionada con esta temática, se ha encontrado que ninguno de los métodos propuestos hasta ahora permiten considerar adecuadamente cómo afecta la gran inercia presente en estos procesos industriales a la hora de negociar la cantidad de electricidad intercambiada a lo largo del tiempo, lo que lleva a que no puedan ser aplicados a una industria como la considerada. Una de las causas más comunes para no incluir las características dinámicas de la planta industrial en el problema de optimización es al alto coste computacional que ello puede suponer. Por ello, en esta tesis se propone como objetivo principal el desarrollo de una nueva metodología para la gestión conjunta de procesos industriales con sistemas de cogeneración acoplados, que facilite la incorporación de dichas características dinámicas y que pueda ser aplicada a las industrias azucareras remanentes tras la crisis del sector. Dicha metodología debe ser extensible a otro tipo de industrias que compartan unas características dinámicas similares.

Así, se propone solucionar dicho problema mediante un enfoque basado en la optimización dinámica, en el que proceso industrial y sistema de cogeneración son considerados de forma simultánea (enfoque integral), de forma que se tiene en cuenta el efecto del acoplamiento energético. Para poder llevar a cabo este enfoque se necesita obtener modelos matemáticos que representen de forma adecuada las características dinámicas del conjunto del sistema (proceso principal y sistema de cogeneración), pero que no supongan una excesiva carga computacional cuando son evaluados. $Y$ es que, al tratarse de un problema de optimización dinámica no lineal, los tiempos de cálculo son rápidamente escalables cuando aumenta la complejidad y el tamaño del modelo utilizado. Se 
propone así la utilización de modelos grises formados por ecuaciones de primeros principios y relaciones empíricas que representan las características dinámicas en lazo cerrado del conjunto del sistema, y que pueden ser obtenidos de una manera sencilla y extrapolable.

Para validar la metodología desarrollada, se ha desarrollado un simulador que representa una fábrica de azúcar de remolacha genérica, y que ha servido como banco de pruebas para probar dos políticas de precios dadas por dos programas de respuesta a la demanda diferentes: Tarifa por tramos, Precios del mercado. En el estudio se ha tenido en cuenta el funcionamiento del mercado eléctrico español, la legislación vigente relacionada con la generación eficiente de energía por parte de los sistemas de cogeneración, y la estacionalidad del proceso de obtención de azúcar de remolacha en España. Para resolver el problema de optimización planteado se ha utilizado un enfoque simultáneo basado en colocación ortogonal, en el que todas las ecuaciones del modelo planteado son discretizadas y resueltas utilizando un algoritmo basado en gradientes como IPOPT. De esta forma se ha reducido la precisión del modelo en favor de una mayor eficiencia computacional.

Como principales resultados del estudio, se ha obtenido que cuando se utiliza la metodología propuesta los costes variables de producción se pueden reducir hasta un $2.55 \%$ si se utiliza una tarifa por tramos típica, y en torno a un $5.41 \%$ si se utilizan los precios dados por el mercado eléctrico directamente. Por supuesto, este último número depende del periodo de tiempo seleccionado para el estudio, pero en general, se puede concluir que cuando se aplica una tarifa con los precios del mercado, los costes pueden reducirse más a costa de asumir un mayor riesgo en el precio. En ambos casos la duración de la campaña propuesta por el optimizador es 3 días mayor respecto a cuando se utiliza un enfoque sin concienciación energética en el que se trata de mantener la producción constantemente al máximo posible.

Por último, conscientes de la dificultad que puede suponer la implementación industrial de una herramienta como la desarrollada, se propone una arquitectura software modular, cuyas comunicaciones están basadas en el standard de comunicación OPC UA. Los tres módulos funcionales que componen el sistema son: una herramienta de optimización desarrollada usando el lenguaje de programación Python ${ }^{\mathrm{TM}}$, un simulador del sistema implementado con el entorno de modelado y simulación Ecosimpro/Proosis ${ }^{\circledR}$, y un sistema de 
supervisión o interfaz de usuario desarrollada con el software Woderware ${ }^{\circledR}$ Intouch ${ }^{\circledR}$, sirviendo esta última como enlace y núcleo del conjunto del sistema. 


\section{Contents}

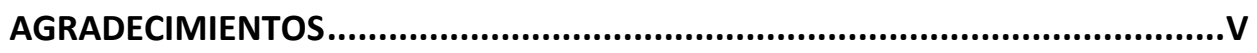

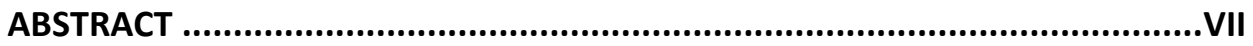

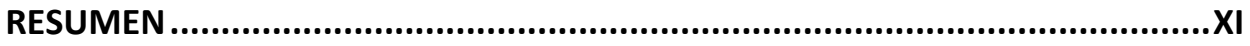

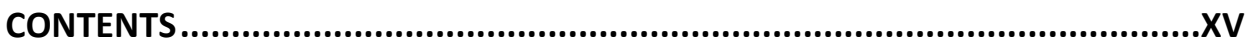

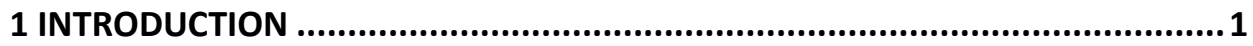

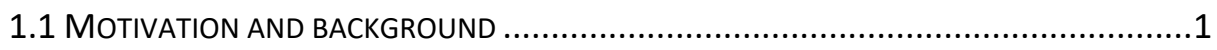

1.1.1 Optimal operation of industrial processes with energy awareness...1

1.1.2 Motivating example: Sugar Industry...............................................

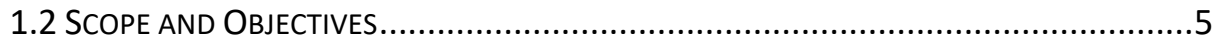

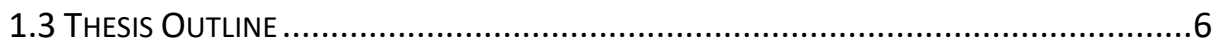

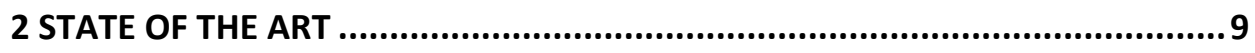

2.1 SCHEDULING OF INDUSTRIAL PROCESSES WITH ENERGY AWARENESS .........................9

2.2 OPTIMAL SCHEDULING OF COGENERATION SYSTEMS IN THE PROCESS INDUSTRY ........17

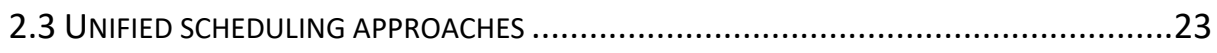

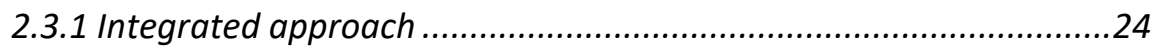

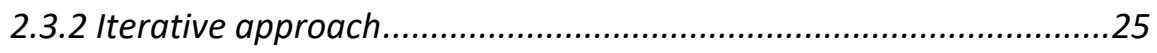

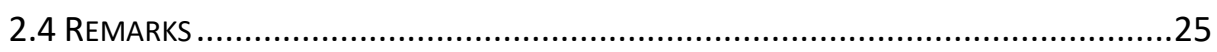




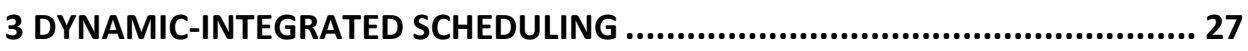

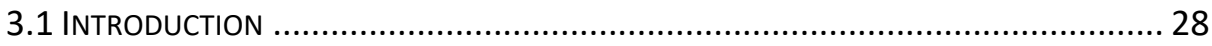

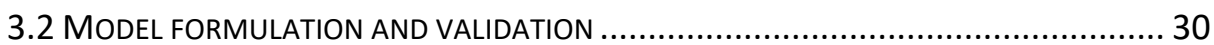

3.3 OPTIMIZATION PROBLEM FORMULATION ..................................................... 34

3.4 INDUSTRIAL ROLL-OUT CONCEPT ........................................................... 41

Operation steps ........................................................................ 43

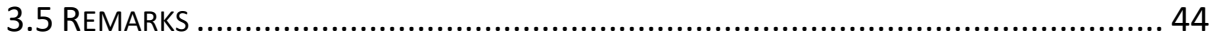

4 CASE STUDY FRAMEWORK ............................................................... 47

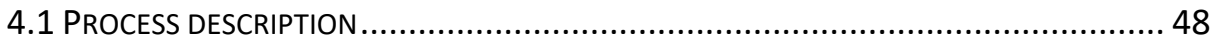

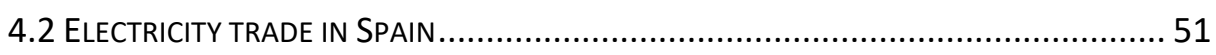

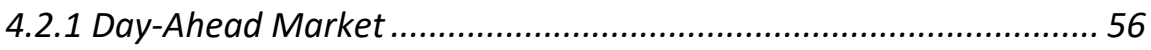

4.2.2 Price formation ................................................................ 57

4.3 LEGISLATION ABOUT COGENERATION PLANTS EFFICIENCY ................................ 58

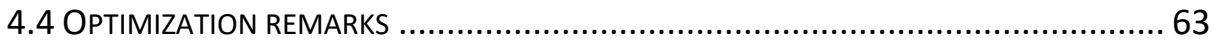

5 INTEGRAL SYSTEM MODEL OF A BEET-SUGAR FACTORY .......................... 67

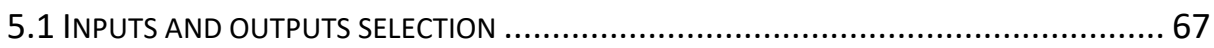

5.2 FIRST-PRINCIPLES EQUATIONS .......................................................... 70

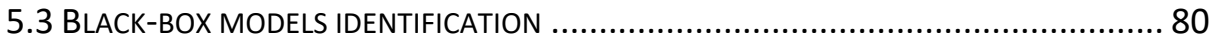

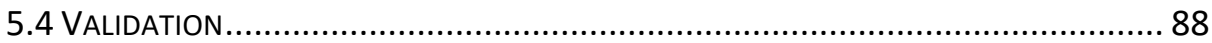

6 OPTIMAL OPERATION OF A BEET-SUGAR FACTORY WORKING WITH A CHP

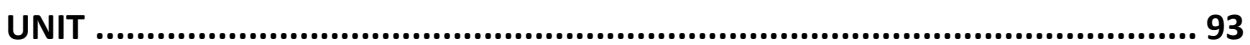

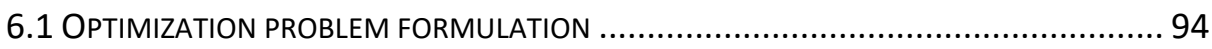

6.1.1 Model reformulation .............................................................. 94

6.1.2 Specific constraints........................................................... 96

6.1.3 Objective function ........................................................... 98

6.2 PENALTY WEIGHTS TUNING .................................................................... 101

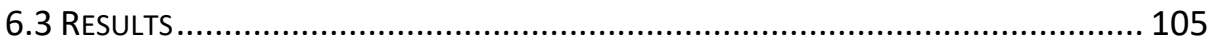

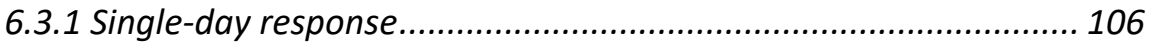

6.3.2 Multi-day response ............................................................ 111

6.3.3 Campaign results................................................................ 113

6.3.4 Computational features ....................................................... 116 
7 FINAL CONCLUSIONS AND OUTLOOK ................................................. 117

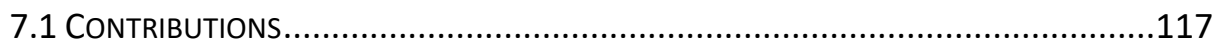

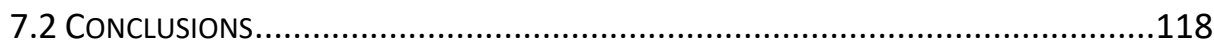

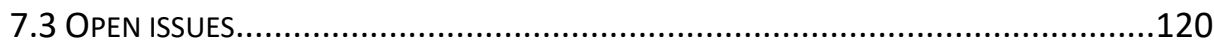

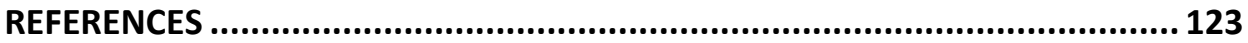

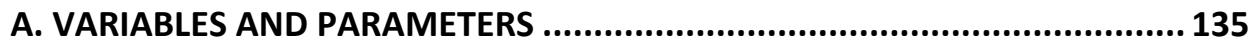

B. INDUSTRIAL ROLL-OUT CONCEPT APPLIED TO A BEET-SUGAR PROCESS .. 141

B.1 ARCHITECTURE ............................................................................ 142

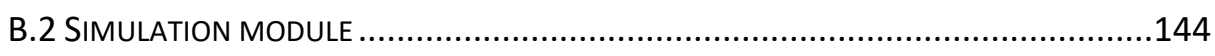

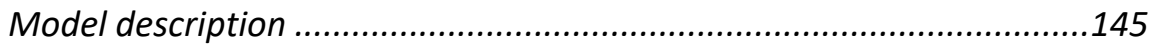

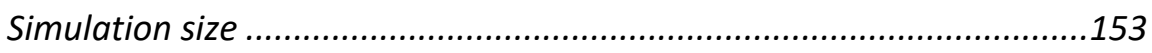

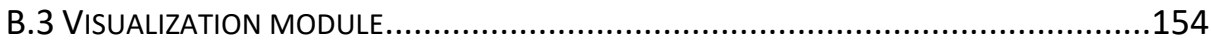

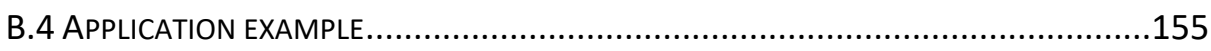

1-Computation of the optimization initial condition. ............................155

2-Computation of the optimization results........................................156

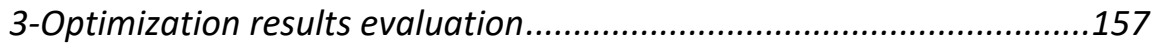

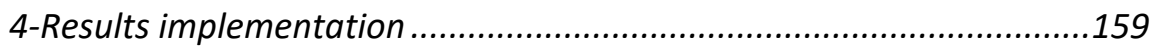





\section{Chapter 1}

\section{Introduction}

\subsection{Motivation and background}

\subsubsection{Optimal operation of industrial processes with energy awareness}

In order to face the present and future environmental problems related to Climate Change, the EU has established the aim of being carbon neutral in 2050. The path for reaching this objective is focused on three key aspects related to energy consumption:

- Reduction of greenhouse gases like carbon dioxide, methane or nitrous oxide.

- Penetration of renewable energies in the final use of energy.

- Increase of the energy efficiency.

Within this framework, the electricity generation system is changing to a decentralized scheme, where new ways of generating and consuming power are rising, giving the final consumer more possibilities than ever. Nevertheless, although this new concept is very convenient from an environmental point of view, features like an intermittent supply of renewable energies, the necessity of new electricity storage systems, or the possibility of self-consumption and dumping to the external grid, may suppose a real challenge to the system 
operator who must ensure the matching of electricity supply and demand in real time.

In this context smart grids rise, where generation, transport, distribution, and consumption are performed in an optimal and coordinated way (Fang et al., 2012). A major innovation of this concept is the consideration of a flexible demand subject to modifications through incentives, topic commonly known as Demand Side Management (DSM) (Meyabadi and Deihimi, 2017). Among the different activities proposed by DSM programs, two different categories can be discerned depending on the pursued objective:

- Energy Efficiency (EE) programs: Aimed to reduce the total energy demand making the same activities more efficient.

- Demand Response (DR) programs: Their objective is to flatten the electricity demand curve changing the end-user electricity pattern. This is crucial in order to avoid the necessity of more electricity generation plants, and the oversizing of the whole electricity system to deal with peak demands.

With respect to DR programs, they can be classified in three different groups attending to the strategy used to modify the consumer behavior (Siano, 2014): Price-based programs, incentive-based programs, and demand reduction bids.

- Price-based programs are composed of non-flat electricity tariffs used to incentive end-users to consume their electricity in non-peak hours. Examples of these policies are: Time Of Use (TOU) rates, where prices are fixed by blocks depending on the time of the day, and Real-Time Pricing (RTP) rates, where consumers are charged with prices that vary over short time intervals, typically hours, and are quoted one day (DayAhead tariff) or less (Intraday and Real-time tariffs) in advance to reflect the real cost of electricity in the wholesale market.

- Incentive-based programs are based on payments done to consumers in exchange of load adjustment capacities given to the grid operator in case of necessity, which can be voluntary or mandatory. Some examples are Direct load control, where the utility has a certain degree of control over some equipment like air conditioning systems, or Interruptible/Curtailable programs, where the customer is asked to reduce its load to a determined value. 
- Demand reduction bids consist in programs where participants send bids to the utility announcing the amount of load they are willing to reduce for a specified price at a certain time. Some examples are Demand Bidding or Capacity Market programs.

Industrial facilities, who require a huge electricity consumption to produce goods and services, are natural candidates for participating in this kind of programs, reducing their operating cost while making the grid more stable and secure. Intuitively, under price-based DR operation, an industrial consumer would increase production (and therefore its power consumption) when the electricity price is lower, storing products in excess and releasing them later when the electricity price is higher. Consequently, in order to participate in such programs, an industrial site must be able to store products safely and efficiently and be capable of adapting its production rate within a time scale similar to the variations in the electricity price. This requires the process to be "flexible", that is, capable of operating in a wide-enough range without incurring in big efficiency losses. Therefore, although at first sight production scheduling and energy management may have competing goals, their optimal integration enables the participation of industrial sites in price-based DR programs, so they have become a topic of interest for a variety of industrial processes in the research community (Albadi and El-Saadany, 2008; Merkert et al., 2015; Siano, 2014; Zhang et al., 2016).

When industrial processes count with on-site generation systems the possibilities are wider, limiting their dependence on the external grid. Cogeneration, or Combined Heat and Power (CHP), is one of the most used onsite generation systems in the process industry. This is mainly because it is a very well-known technology, and it provides much higher efficiency values than traditional generation systems thanks to the simultaneous production of heat and power from only one source of energy (Kehlhofer et al., 2009).

The operation of industrial processes and their associated cogeneration plants is usually coupled at a certain point, meaning that the heat and electricity production cannot be considered independently of the main process operation. The independent management of both systems will lead in most of the cases to suboptimal or even infeasible operation strategies. Thus, in this thesis we propose such coordination based on a mathematical optimization approach, 
obtaining feasible operation policies while exploiting the possibilities raised by price-based DR programs.

\subsubsection{Motivating example: Sugar Industry}

In 1968, under the Common Agricultural Policy (CAP), the European countries reached an agreement regarding sugar production with two essential principles:

- Ensuring a preestablished price for agricultures.

- Self-sufficiency inside the Union.

Thus, to deal with the high costs related to sugar production, an expensive sugar price was fixed, being three times higher than in the international market. If the price went below the preestablished one, the European Union was forced to buy and, to avoid the excessive importation, big taxes were imposed to foreign sugar.

Given the appeal of this business and the possibility of overproduction, a quota system was installed inside the borders. Thus, each European country was restricted to produce a determined amount of sugar per year and, if this quantity was surpassed, they were forced to save it for the next year or export it to the international market. Given that if industries saved the sugar excess, they would be forced to reduce their production for the next year, most of the surplus was sold. However, instead of selling it for the same price as inside Europe, they did it for a price twice or even three times cheaper, what caused a collapse in the international price. This practice is known as dumping, and it was supported by the high prices paid by European consumers.

In 2005, Thailand, Australia, and Brazil reported the bad practices of the European Union to the World Trade Organization, which sentenced that the market inside the EU had to be liberalized. This would be done progressively till 2017 to enable the sector adaptation. The reforms had three main objectives in mind, firstly, to increase the world sugar price, secondly, make it more stable, and lastly, to improve the working conditions in growing countries.

In October 2017, the complete liberalization of the market arrived. The consequences varied depending on the countries, for example, in France and Germany the production increased, while in others such as Portugal or Ireland it disappeared. Regarding the European price, it has experienced a big drop during 
the last years, which has been the cause of many sugar factory closures and dismissals (Castro and Villadiego, 2013).

In order to survive, beet-sugar companies in countries like Spain have realized that they need to be as competitive as possible. However, sugar is a commodity, i.e., it is a basic highly spread product. Such industries can hardly innovate in its production to differentiate themselves from the competence, and therefore, any improvement, even a small one, is an important achievement. Hence, many sugar industries have looked for different solutions in order to reduce costs or increase profits. Among the different alternatives found, stand out: Ethanol generation, animal feed production and electricity generation (Lora et al., 2013).

Cogeneration systems are usually part of the classical configuration of these industries, therefore, in this thesis, the coordination of sugar production and electricity generation has been researched in order to reduce the sugarextraction operating costs. Given the possibilities raised by the price-based DR programs, an algorithm that helps to operate the process considering different tariffs is proposed in order to reduce the costs related to sugar extraction from beet.

\subsection{Scope and Objectives}

\section{MAIN OBJECTIVES:}

- Propose a new methodology, based on dynamic optimization techniques, to manage the production of industrial processes and cogeneration plants, both coupled from an energetic point of view and connected to the external grid. Such management will be guided from economic objectives that include the electricity price given by pricebased Demand-Response programs, constraints imposed by the production process, and the current legal framework.

- Specify and apply such methodology into the Spanish beet-sugar extraction industry, contributing to its competitiveness and to the stability and safety of the power grid. 


\section{SPECIFIC OBJECTIVES:}

- Study the current situation and advances in terms of methodologies and techniques used to manage the operation of industrial processes and cogeneration plants with energy awareness.

- Understand and document the functioning of the Spanish electricity market and the current legislation regarding the efficient operation of cogeneration systems.

- Propose a generic methodology to obtain and update mathematical models of the system formed by industrial process and cogeneration plant. Such models must be suitable for dynamic optimization techniques, keeping a balance between accuracy and computational complexity.

- Formulate the dynamic optimization problem in an efficient way, so sufficient time is available to evaluate the proposed solutions given by the optimizer.

- Develop a dynamic simulator of a generic case study, where the proposed methodology can be tested. Specifically, a beet-sugar extraction process working with a natural gas cogeneration plant connected to the external grid.

- Validate in silico the proposed methodology using two typical demand response programs and compare the results with a strategy without energy awareness, where the production is kept at maximum values for the whole season.

- Propose a software architecture for the implementation of the developed optimization tool, based on supervision tools used in industry. Test its performance in simulation.

\subsection{Thesis Outline}

After Chapter 1, in Chapter 2 the state-of-the-art regarding the optimal operation of industrial processes working with and without cogeneration systems considering energy awareness is studied. In this chapter we analyze the work done regarding this topic so far, and we outline some gaps detected.

In Chapter 3, we present the Dynamic-Integrated scheduling approach, used to solve the optimal operation of industrial processes operating with 
cogeneration systems when the dynamic features of the system must be considered. Also, we expose the assumptions done to apply such methodology, and we discuss its limitations.

Chapter 4 describes the case study framework considered to test the methodology described in Chapter 3. First, we give a description of the simulated beet-sugar extraction process, then, we analyze the Spanish electricity market, and finally, we explain the European legislation regarding the efficient use of cogeneration systems.

In Chapter 5, we apply the first part of the methodology proposed in Chapter 3 , concerning the derivation of gray-box mathematical models of industrial processes operating with coupled cogeneration systems, to the case study described in Chapter 4. Thus, we obtain a simple but balanced dynamic model of the system, ready to be used for optimization.

Chapter 6 is focused on the optimization stage and describes how to formulate the optimization problem for the case study considered. Furthermore, in this chapter, we compare the performance of our approach using two different price-based demand response programs, with respect to operating with a base tariff and a non-energy awareness policy during a whole campaign.

In Chapter 7, first, we highlight the contributions of this thesis, then we give some general conclusions about the work done, and finally, we discuss some open issues that could be carried out as future work.

Finally, in Appendix A we list all the variables and parameters used in this work, and in Appendix B we apply the software architecture concept presented in Chapter 3 to our case study. 



\section{Chapter 2}

\section{State of the art}

In this chapter, we study the current status of the research related to the optimal operation of industrial processes working with cogeneration systems. In the first section, we give a vision from the industrial process perspective reviewing the optimal scheduling of industrial processes with energy awareness. Thus, we explain the concept of scheduling in process industry, and we show the importance of considering the system dynamics in the optimal operation calculations, reviewing the most important approaches used in the literature. In the second section of this chapter, we start taking a different perspective, specifically the cogeneration point of view. First, we talk about the sequential approach, which is the most extensive solution used in the literature to optimize the operation of CHP systems using a master-slave architecture. Later, we review the integrated approach, where both systems are considered at the same time, and we end the section giving some ideas about the iterative approach. To conclude this chapter, in the third section, we explain the reasons why we consider that more research is needed in this area, giving the justification for our research.

\subsection{Scheduling of industrial processes with energy awareness}

In the Process System Engineering literature, we can find a variety of works that study how to operate chemical processes according to Demand Response 
programs, an extensive review can be found in (Zhang et al., 2016). While this study includes all kind of DR programs, we have centered our research in those works who study the implementation of price-based DR programs. We have found examples of implementation in the following industries: Flour/Pulp production (Ashok and Banerjee, 2001), seawater reverse osmosis (Ghobeity and Mitsos, 2010), steel (Pan et al., 2019; Zhao et al., 2018), machining (Yusta et al., 2010), chlor-alkali (Brée et al., 2019), cement (Castro et al., 2011; Mitra et al., 2012), and air separation (Caspari et al., 2019; Kelley et al., 2018; Mitra et al., 2012; Pattison et al., 2016; Pattison and Touretzky, 2016; Schäfer et al., 2019; Tsay et al., 2019; Zhu et al., 2010). In Table 2-1 we list the papers reviewed for this section, the industry studied, and the price-based DR program implemented in each case study.

The problem posed in all these works can be generally defined as follows: Given the electricity price forecast, the product specification, and demand for a certain period, determine the best process scheduling that optimizes the operating cost or profit, while ensuring some operational constraints. To solve this problem first we need to understand the hierarchy of decisions in industrial process operation, which is illustrated in Figure 2-1.

One of the main differences between the different levels of the hierarchy is the time scale of the decisions taken. In the planning layer, decisions are taken for a period of months. In this layer, the objective is to meet the contractual agreements made with clients based on product quantities and specifications. This problem is solved by the business department, and yields the demand forecast that must be met by the scheduling layer. 
Table 2-1. List of papers reviewed for this section ordered chronologically.

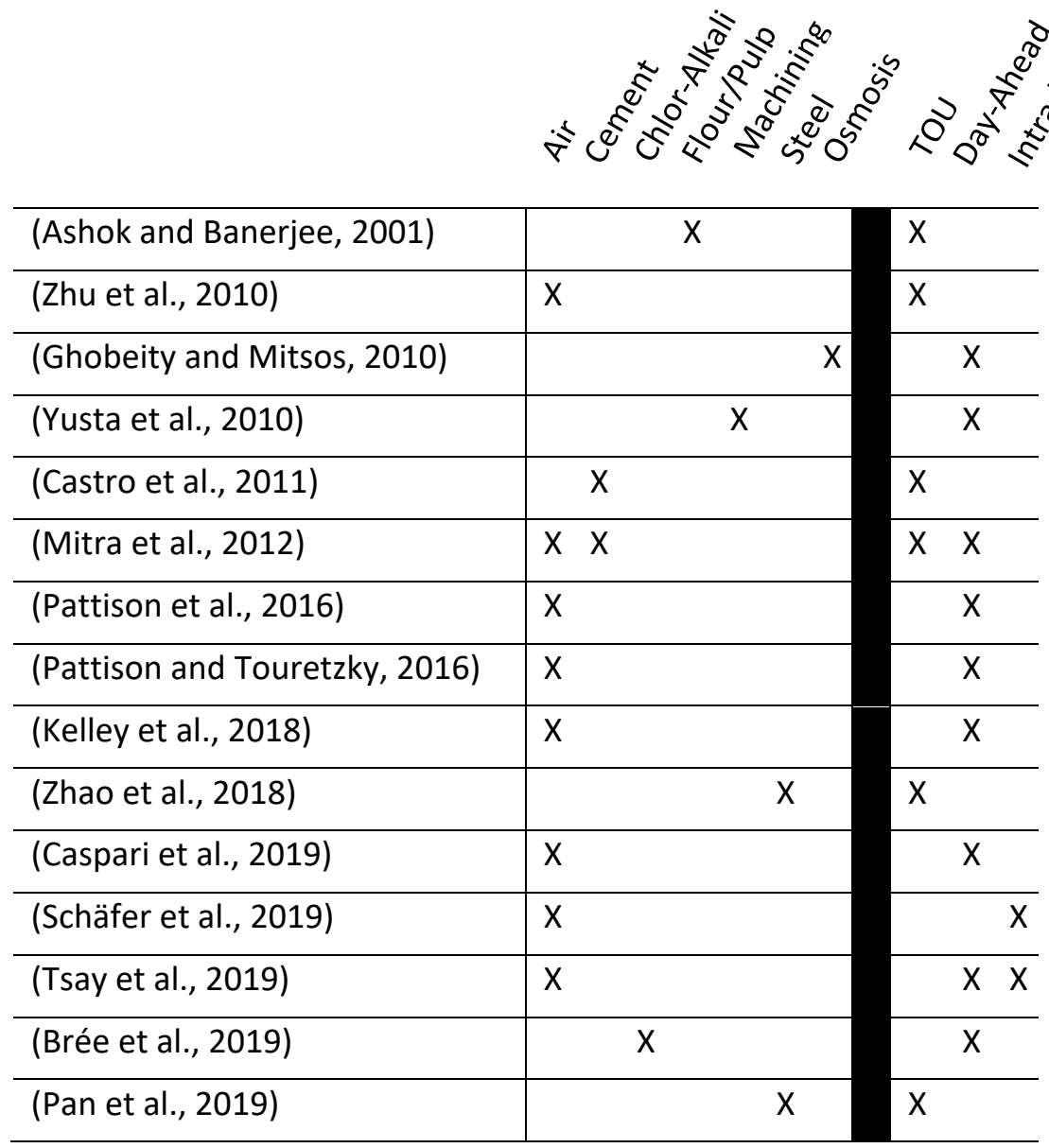

One of the most usual objectives of the scheduling problem is to find the best production plan that maximizes profit while meeting the demand forecasted. The plan computed must be consequent with the capacity restrictions and inventory levels of a determined factory. This is an optimization problem where the time scale goes from hours to days, and gives an answer to the following questions (Harjunkoski et al., 2014):

- Distribution of the production orders? (Batching)

- What task to execute? 
- Where to process the production tasks? (Assignment)

- In which sequence to produce? (Sequencing)

- When to execute the production tasks? (Timing)

Depending mainly on the process type (batch vs. continuous) and the production environment (sequential vs. network), the output of this layer will be a set of continuous and discrete variables that will be sent to the control layer.

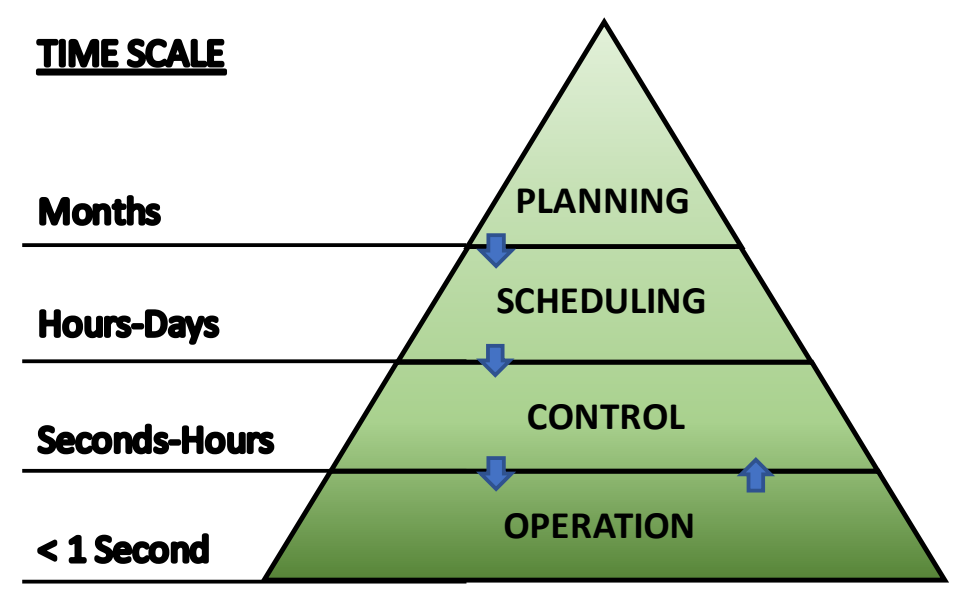

Figure 2-1. Decision hierarchy in process operation.

The goal of the control problem is to find the control law that ensures that the process outputs are as close as possible to the desired values imposed by the scheduling layer in the presence of potential disturbances while respecting different operational constraints. This layer interacts directly with the production process equipment and defines the dynamic response of the process. Its time scale goes from seconds to hours depending on its complexity, and its key feature is the presence of feedback from the operation layer, where the decisions computed by the control layer must be executed as fast as possible. It comprises the physical devices existing in industry, such as actuators and sensors.

Traditionally, scheduling problems have been solved manually by plant engineers using their experience. However, due to the complexity of this task it is very difficult to ensure a profitable production without any mathematical optimization support. That is why in recent years, many researchers have been working on how to solve these optimization problems in the best possible way, 
improving things like the formulation, software and solvers employed. A complete review about this topic can be found in (Harjunkoski et al., 2014).

Conventional methods for finding the optimal production schedule use stationary mathematical process models, where the transition behavior between different operating points and/or modes is neglected or simplified using tabulated values or defined functions. Besides, usually, they assume that the process is at a steady state before a change in the production target is done, and that it reaches a new steady state before a new change is performed. This has been the approach taken in (Ashok and Banerjee, 2001; Brée et al., 2019; Castro et al., 2011; Ghobeity and Mitsos, 2010; Mitra et al., 2012; Zhu et al., 2010).

While this approach may be enough for many case studies, depending on the process and the DR program selected, the electricity prices may change with a frequency that is comparable to the dominant dynamic of the process. If that is the case, simplifying transition times may lead to compute a suboptimal or even infeasible sequence of transitions in the scheduling layer (Tsay et al., 2019). This problem is illustrated in Figure 2-2.
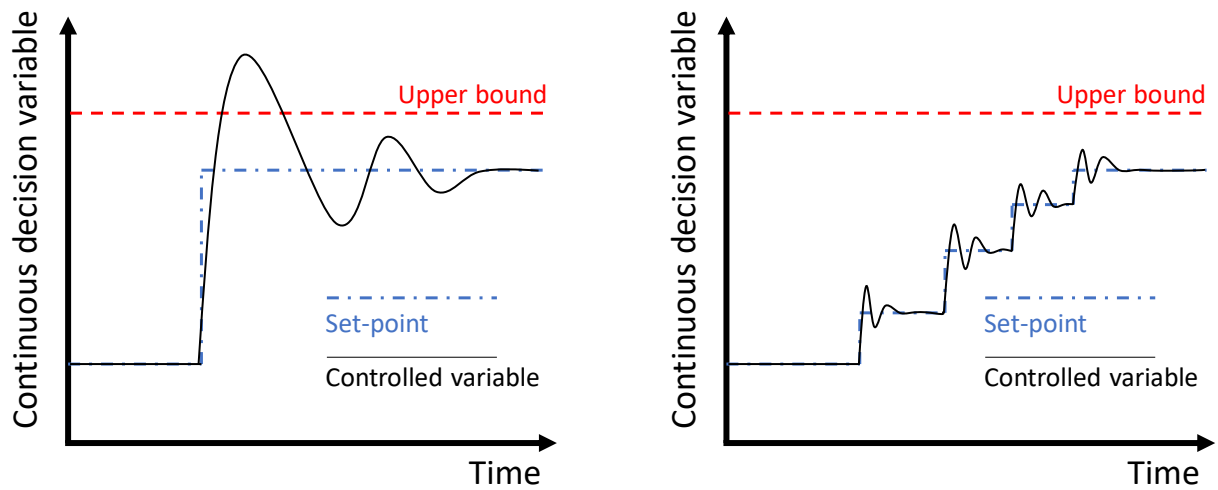

Figure 2-2. Illustrative example of a potential unfeasible solution that may arise from not considering dynamics in a scheduling problem.

In Figure 2-2, on the left side, we can see that if the dynamic of the process is not represented correctly in the scheduling layer, the optimizer will try to reach the new operating point as fast as possible. This may cause that some important variables exceed the limits imposed in the optimization problem without being noticed by the optimizer, which will lead to quality or safety production problems. On the right side of Figure 2-2, the reader can see that now the 
optimizer considers the real dynamics of the process, and therefore, to reach the same operating point it suggests smaller changes, sufficiently spaced, so the bounds are not violated.

The use of dynamic information for making scheduling calculations falls under the category of integrated scheduling and process control. A complete review of this topic may be found in (Baldea and Harjunkoski, 2014). Ideally, a full integration of the scheduling and control problems would lead to the best sequence of operating points that fulfills an economic objective, while the dynamic of the plant would not be only known, but also adapted in order to reach such points in the shortest possible time.

Following the discussion about this topic, in (Baldea and Harjunkoski, 2014), there are two different ways to deal with this problem: Top-down approaches, which refer to consider dynamics and control elements in a scheduling problem formulation, and bottom-up approaches, where the controller layer itself accounts for the scheduling problem.

Regarding top-down approaches, there are three different ways for adding dynamic information to the scheduling layer:

- Control-defined transition times. Transition times are defined based on a typically explicit control law. This way, for example, the optimal scheduling together with the best parameters of a PID controller may be calculated offline, and later, both can be implemented online at the same time.

- Dynamic optimization-based scheduling. In this case, a full-scale dynamic model of the process is implemented in the scheduling calculations, and typically, a Mixed-Integer Dynamic Optimization (MIDO) problem will have to be solved to obtain the best production sequence and the optimal control moves.

- Time-scale bridging. It consists in using a low-order representation of the dynamics of the process and its control system, so they can be considered in the scheduling problem but not modified. Therefore, a closed-loop model that represents the dependence of the process outputs with respect to the inputs is used instead of the whole dynamic model. This idea comes from multi-scale simulation, and it is a natural way for dealing with the different time scales considered in the scheduling and control problems (Du et al., 2015). 
Depending on the complexity of the system considered, each approach has its pros and cons. While control-defined transition times are the simplest way to introduce control information inside the scheduling layer, sometimes, this is not enough to represent all the dynamics needed in the plant, and they depend on the use of known explicit control laws, so more sophisticated control options are discarded. Dynamic optimization-based scheduling solves both problems, but at the expense of introducing an important amount of complexity in the model, which in many cases will make the problem very difficult to solve. Both strategies have the disadvantage of calculating the control sequence offline, so the process operation is subject to disturbances and modeling mismatch. Some authors have proposed the possibility of using a rescheduling approach, where the scheduling is recalculated periodically (Zhuge and lerapetritou, 2012). While, in general, this solution can be easily implemented when control-defined transition times are used, it exacerbates the computational problem found when using full-scale dynamic models.

Time-scale bridging arises as a trade-off solution between detailed solutions and computational complexity. Using closed-loop low-order models introduces in the scheduling problem enough information of the dynamics of the plant to avoid infeasible operation strategies, reducing the complexity of MIDO calculations, at the expense of not optimizing the dynamic behavior of the plant, which is still managed by an independent control layer. For the optimal management of industrial production according to electricity prices, this approach has been recently studied and compared to other solutions in (Kelley et al., 2018; Pattison et al., 2016; Pattison and Touretzky, 2016; Schäfer et al., 2019; Tsay et al., 2019).

On the other hand, the most developed bottom-up approach is Economic Model Predictive Control (EMPC), where the typical tracking objective function found in a Model Predictive Controller (MPC) is substituted by an economic one (Ellis et al., 2014; Rawlings and Amrit, 2009). In this approach, the economic optimization problem is solved at the control layer using an open-loop dynamic process model, which enables the possibility of solving the scheduling and control problem at the same time. Compared to bottom-up approaches, exact constraints can be used directly, it presents feedback mechanisms for ensuring closed-loop stability in the presence of disturbances, and given that only one layer is used, feasibility of the process decisions computed in a higher layer is not 
an issue. Other important advantage is that extensive theoretical foundation about this approach regarding aspects like stability, convergence or performance can be easily found in the literature.

However, with this approach a dynamic optimization problem must be solved online. This is an important issue given the mathematical complexity needed to represent the full process behavior of many industrial applications. In (Schäfer et al., 2019) some model reduction approaches were mentioned, and they proposed the use of data-driven models for the optimal operation of an air separation process in real-time electricity markets. Although this is a promising approach, a lot of work is still needed in this topic to ensure accurate-enough real-time solutions when more complicated processes are studied. In the same line, given the higher complexity of the formulation proposed, the use of discrete decision variables has received little attention in this context so far. Some examples of the use of EMPC for electricity consumption management of industrial applications can be found in (Caspari et al., 2019; Schäfer et al., 2019). The rolling-horizon nature of EMPC makes its application interesting in problems where operational decisions can be taken several times within a time horizon. This is the case if a real-time tariff is considered, but for other options, like a DayAhead one, decisions can only be taken once a day, so top-down approaches become more interesting.

For a full comparison between the top-down and bottom-up performance, the reader is referenced to (Caspari et al., 2020). In Figure 2-3, a scheme of the approaches reviewed in this section to deal with the integrated scheduling and control problem is presented. 


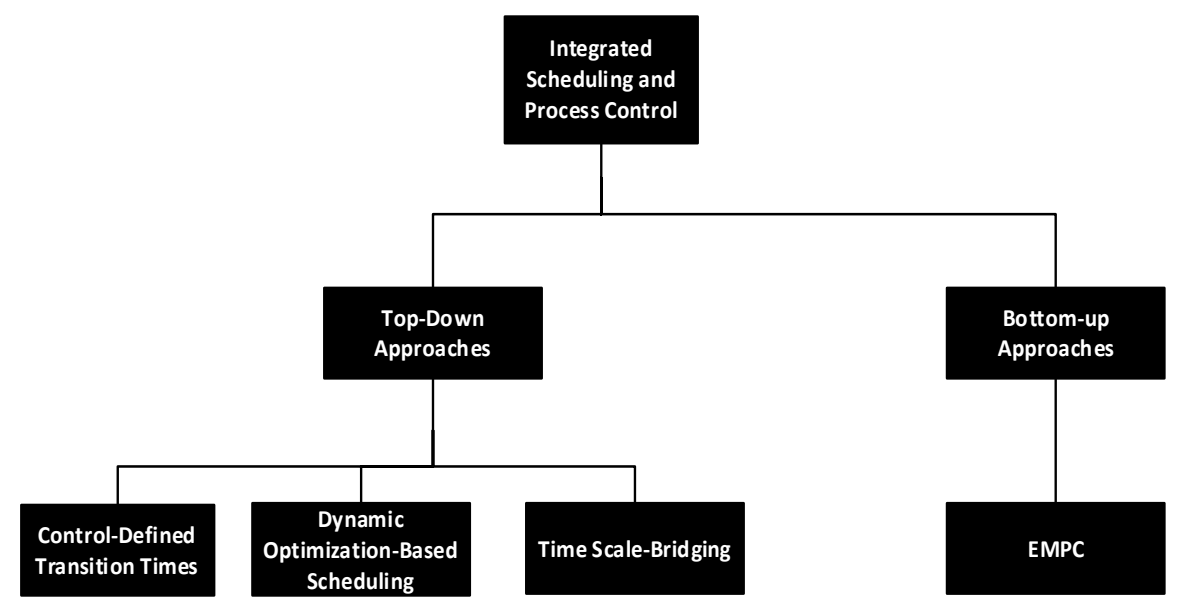

Figure 2-3. Scheme of the most-used methodologies used to solve the integrated scheduling and control problem.

\subsection{Optimal scheduling of cogeneration systems in the process industry}

In the previous section we reviewed the optimal operation of industrial sites with energy awareness. While some of the case studies shown in Table 2-1 counted with auxiliary systems to generate their own energy, like cogeneration plants, they did not consider them in detail in the optimization problem. Thus, the classic strategy is, first, to solve a scheduling problem of the industrial process production that generates some energy needs, and later, solve a new optimization problem, where the auxiliary plant must optimize its operation to fulfill such energy requirements while minimizing the associated operating costs. In this section, we put our attention in this problem focusing on the operation of cogeneration systems, where decisions can be taken at two different levels:

1) Cogeneration plant level. Decisions are related to the quantity of electricity that must be bought from the external grid or generated onsite in order to sell a surplus if possible. Here, different price-based Demand Response programs are usually considered, and the price of the electricity takes on a special relevance.

2) Equipment level. Once the quantity of electricity to be generated has been decided, the next step is to decide which equipment is going to produce it; this is known as the Unit-Commitment (UC) problem. Then, the load generation of each of the selected pieces of equipment is decided (Economic Dispatch (ED) problem) (Conejo and Baringo, 2018). 
The optimal solution of the CHP short-term scheduling has attracted much attention from the research community during the last years. In (Salgado and Pedrero, 2008), the authors presented the three main research lines regarding this topic (Figure 2-4): Those that focus on new operation strategies, those that give importance to the optimization model, and those that present new algorithms for solving the ED and UC problem.

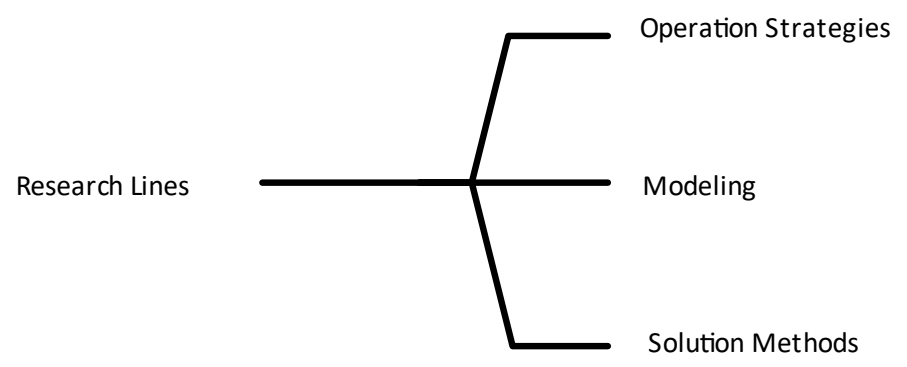

Figure 2-4. Optimal CHP operation research lines in the sequential approach.

\section{Operation strategies}

Between the different operation strategies found in the literature (Salgado and Pedrero, 2008), we have put our attention in those works that exploit the possibilities given by different electricity tariffs and markets trading power with the external grid. This was the case in (Yusta et al., 2008), where the authors proposed an optimal strategy for a real combined-cycle cogeneration of an industrial factory that sold power in the Spanish Day-Ahead electricity market and purchased it through a retailer. (Tina and Passarello, 2012) proposed a strategy that aimed to maximize the revenue of large industrial sites that counted with cogeneration plants, trading electricity in the day-ahead Italian market and heat through long-term contracts. They paid special attention to the compliance of the legislative constraints imposed by the Italian Government in order to get special advantages. Also focused on the legislation applied to cogeneration, we found (Gambini and Vellini, 2014) who explained how to apply the European Legislation to CHP plants, and (Gvozdenac et al., 2017) who gave some practical procedures to recognize the CHP and non-CHP parts of a cogeneration system, showing how to apply them in a particular case study. 


\section{CHP modeling}

To formulate the optimization problem, a model that describes the CHP system is necessary. A good model is key in order to obtain a good accuracy with an affordable computational effort. In the literature we can find two main approaches for modeling CHP systems (Dvořák and Havel, 2012): Black-box models, and first-principles equations. Black-box models are used to simplify the relationship between the power and heat generated in a CHP plant. This way, if one of them is known, the other one can be obtained directly. This relationship has been found in the literature using: Feasibility regions, power-to-heat ratios, and data interpolated expressions. Feasibility regions are represented in a power vs heat plane, an example obtained from (Salgado and Pedrero, 2008) can be found in Figure 2-5. They can be convex (Sashirekha et al., 2013), non-convex (Shaabani et al., 2017) or both simultaneously (Kia et al., 2017). Other works used a power-to-heat ratio instead. Some researchers have considered it to be constant, which depending on the case studied may be a gross approximation (Frangopoulos, 2012). In other studies like (MacGregor and Puttgen, 1991), the authors presented an approach where the ratio varied between some fixed upper and lower bounds, and in (Santos and Uturbey, 2018), it was presented as a function of the efficiencies of the equipment. Models based on data interpolation with polynomial functions, were used by (Tina and Passarello, 2012).

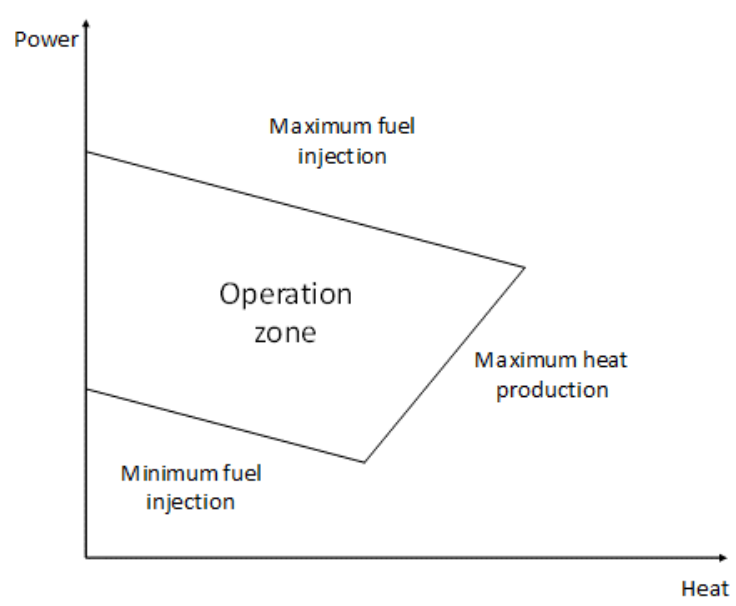

Figure 2-5. CHP feasibility region example. 
Instead of black box models, an alternative is the use of detailed firstprinciples models to describe the CHP systems. Some examples are (Bindlish, 2016; Bruno et al., 1998; Godoy et al., 2011; Kim and Edgar, 2014). (Kim and Edgar, 2014) presented the scheduling of a cogeneration plant in the day-ahead electricity market using detailed models to describe the CHP plant with different operating modes. They tested their approach in a power plant complex at the University of Texas. In (Bindlish, 2016), a scheduling application was proposed using a steady-state, first principles, nonlinear model. The author in this case presented a sequential approach, where first a scheduler computed the power that had to be offered in the United States real-time market, and then it was implemented using a model predictive control approach. The use of stationary models was justified considering that the dynamics of power generation in the CHP plants are much faster than changes in the electricity price. In all these cases, more precise and general solutions were obtained at the expense of a greater computational effort. The main computational problem in first-principles equations comes from the nonlinear nature of the equipment efficiencies, which depend on changes in the enthalpy. To reduce the computational time while maintaining the model complexity, three different approaches were found in the literature: Efficiencies were treated as constant in (Marshman et al., 2010), a piece-wise linear function was used in (Dvořák and Havel, 2012), and an inputoutput estimation based in thermodynamic concepts like the Willans line was implemented in (Ashok and Banerjee, 2003). Commonly, each equipment is treated independently, so the three approaches can be used simultaneously (Agha et al., 2010; Yusta et al., 2008). A summary of the approaches described to model a CHP system is given in Figure 2-6. 


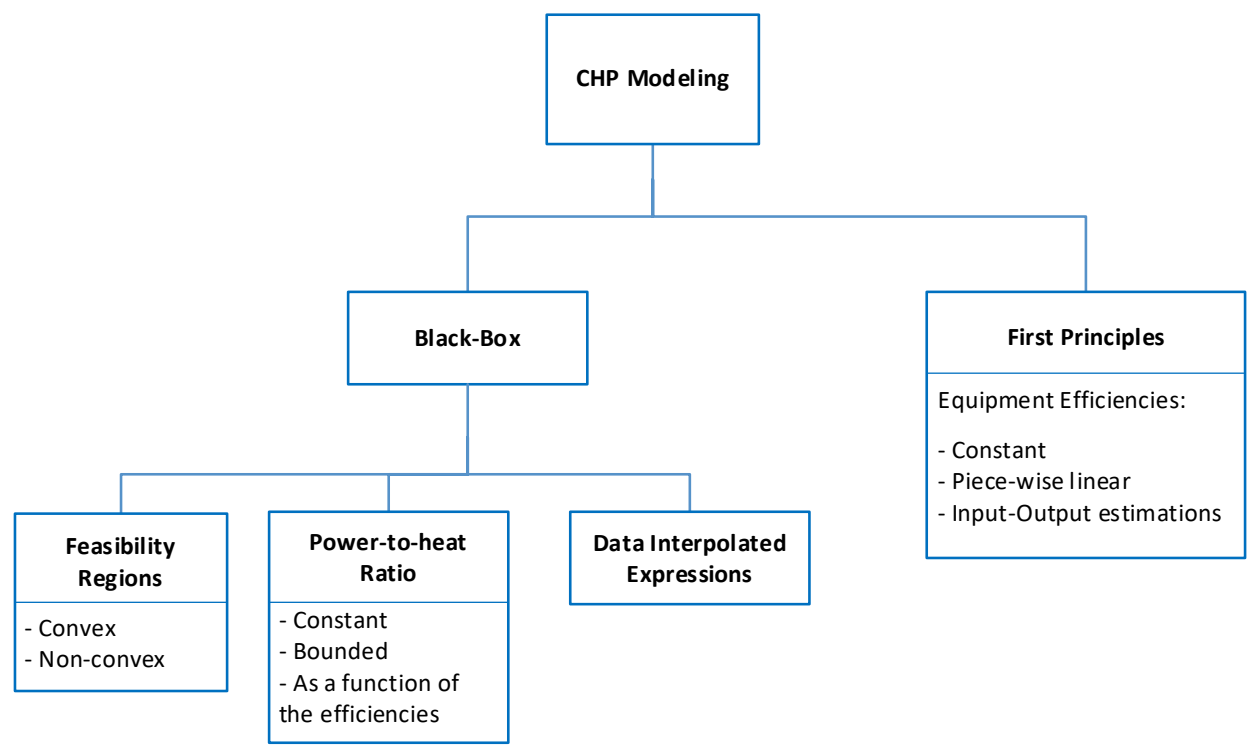

Figure 2-6. Summary of the CHP modeling approaches.

\section{Solution Methods}

The formulation of the unit commitment problem is usually addressed as a mixed-integer programming problem (MIP) (Floudas, 1995), and the economic dispatch as a non-linear programming problem (NLP) (Biegler, 2010). When both problems are solved at the same time, nonlinearities are usually simplified, and a mixed-integer linear programming problem (MILP) is proposed to avoid numerical difficulties. This way, the integer values are used to select between different operation modes and equipment, and the real variables to evaluate costs and decide the energy that must be generated in each unit. For example, (Mitra et al., 2013) addressed the scheduling of industrial CHP plants using mixed-integer linear programming considering different operating modes and transitional behavior. They also used disjunctions to solve the problem faster. A more detailed model was proposed in (Venkatesh and Chankong, 1995), where a tool was presented for optimal management of cogenerations in industrial or commercial settings. To linearize the model, they used data from physical tables to obtain enthalpies, and they assumed that the equipment efficiencies were constant. Sometimes a multi-objective formulation is proposed instead, where usually, polluting emissions are considered as a second objective. This was the 
case in (Aghaei and Alizadeh, 2013), where the optimal operation of a CHP-based microgrid considering DR programs was presented as a multi-objective problem, where the first objective was the minimization of the total operating costs and the second referred to emission minimization.

Traditionally, the optimal scheduling of CHP plants is a mathematical problem solved offline, whose solution is sent later to the control layer. For this problem, if the electricity is decoupled from the thermal generation using technologies like heat storage systems (Christidis et al., 2012), transition times are usually neglected, considering that changes in the CHP operating conditions are much faster than changes in the electricity prices. Nevertheless, if the intraday market is considered, that is not necessarily true, and some recent works studied the possibility of solving this problem with an Economic Model Predictive approach using a control-based strategy. This was the case in (Diaz C. et al., 2019), where the authors solved the optimal operation of a real CHP plant participating in the German intraday market. The authors proposed a nonconstant time step to get a better resolution at the beginning of the horizon using a shorter step and a larger one for the far instants.

Furthermore, uncertainty is always present in the operation of CHP systems. Given the characteristics of the day-ahead electricity market, a bad prediction of the consumers electricity or heat demand may lead to deviations with respect to the commitment done and, hence, to high penalties. For example, in (Majidi et al., 2019), the optimal operation of CHP systems was studied under the effect of the uncertainty brought by Demand Response Programs, and solved with robust optimization. The market price uncertainty is also a very important factor when bidding in any electricity market. In (Alipour et al., 2016), the authors studied the optimal bidding strategy in the day-ahead energy market of industrial cogeneration systems using an information gap decision theory.

Depending on the mathematical formulation selected, different solution methods have been used in the bibliography to solve the sequential approach. They can be classified as: Classical mathematical programming methods and heuristic optimization procedures. Among the classical mathematical methods used in the literature we found: Newton's method (Ashok and Banerjee, 2003), Benders decomposition (Sadeghian and Ardehali, 2016), Lagrangian relaxation (Sashirekha et al., 2013), branch and bound algorithm (Rong and Lahdelma, 2007), and mixed-integer nonlinear programming methods (Kim and Edgar, 
2014). Regarding the heuristic optimization procedures, they are experiencebased optimization methods used when the classical mathematical approaches fail to provide a solution in a finite amount of time. Some examples are: Genetic algorithms (Subbaraj et al., 2009), particle swarm optimization (Piperagkas et al., 2011), differential evolution (Jena et al., 2016), group search optimization (Basu, 2016), etc.

\subsection{Unified scheduling approaches}

In the literature we can see that the most extended approach in the process industry to deal with the operation of cogeneration systems is the sequential one, where the problem is separated into two subproblems where the solution of the first one is sent to the second one. This is the one reviewed in the previous section. The main advantage of this approach is that for realistic problem sizes the computational cost is relatively low, but the solution usually leads to suboptimal or non-feasible points.

One alternative is the use of a strategy where the solution of both problems is considered at the same time. This is the case in the integrated approach, which leads to better solutions at the cost of a higher computational effort.

Furthermore, we can find a trade-off solution in the iterative approach, where both problems are optimized independently but exchanging information. Thus, the solutions will be better than the obtained in the sequential approach, while spending less time that in the integrated approach, and avoiding nonfeasible solutions. A summary illustration of the three approaches obtained from (Hadera et al., 2019) is shown in Figure 2-7.

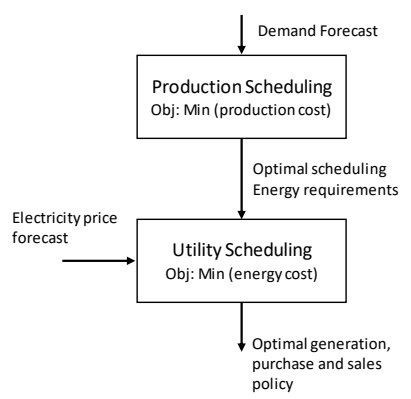

a) Sequential approach

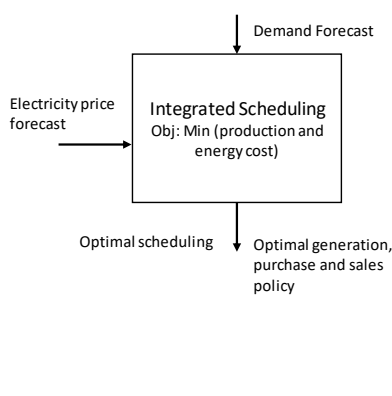

b) Integrated approach

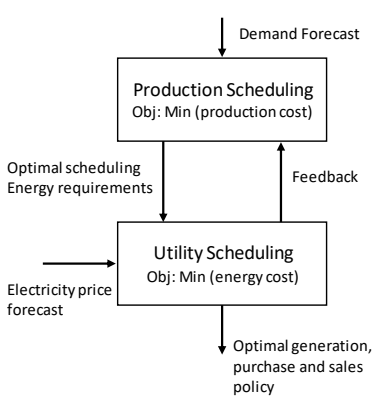

c) Iterative approach

Figure 2-7. Approaches for solving the scheduling of production and cogeneration units. 


\subsubsection{Integrated approach}

As explained in the previous section, the sequential approach considers the cogeneration and industrial operation independently. In many cases this will lead to suboptimal solutions because of the strong dependency between both systems. When the integrated approach is considered, energy generation and consumption is optimized at the same time. In this section, we show some examples where the integrated approach was taken.

One of the first works found in the literature where the idea of integrating process and utility operation for a better operation appears is in (Moita et al., 2005). In this work the authors developed a dynamic model of a cogeneration system integrated with a salt recovery process and studied its optimal operation via simulation. Later in (Zhang and Hua, 2007) an MILP model was developed for the production planning of an oil refinery industry coupled with a cogeneration unit. In (Agha et al., 2010) the integrated approach was defined, and the authors compared its performance against the sequential approach, concluding that the first one leaded to significant reductions in the energy costs while decreasing the emissions of harmful gases for the case studied.

Later in (Wang et al., 2013) the idea of optimizing the participation of energy intensive processes with on-site generation systems in Demand Response programs was studied. Thus, the implementation of a TOU tariff along with a load tracking problem was proposed. In (Ding et al., 2014) a DR program based on day-ahead hourly electricity prices was implemented with a MILP model. On the other hand, (Zhang et al., 2013) studied the use of a mixed-integer nonlinear programming model to solve the integrated approach in two real refineries coupled to cogeneration systems. A similar approach was taken in (Sun et al., 2015), although in that case an heuristic method (Particle Swarm Optimization) was used to solve the optimization problem. A bi-level heuristic problem was proposed in (Hadera et al., 2015) to optimize the melt shop section of a stainless steel plant. In this study, the authors considered different sources of power and the possibility of selling the electricity back to the external grid.

(Zulkafli and Kopanos, 2016) proposed a model where performance degradation and different types of cleaning were considered for the utility system. Later in (Zulkafli and Kopanos, 2017) they extend their approach to propose a rolling horizon optimization to cope with demand uncertainty. 
Equipment breakdowns and deviation price uncertainty was considered in (Leo and Engell, 2018). In this work the authors presented a stochastic mixed-integer linear programming model to simultaneously determine the optimal production schedule of a power-intensive plant with on-site generation capabilities and the optimal day-ahead electricity commitment.

\subsubsection{Iterative approach}

The main drawback of the integrated approach is the bigger computational effort needed to solve the problem with respect to the sequential approach. To deal with this problem and take advantage of the problem structure, an iterative strategy, also called a decomposition approach, has been recently proposed by some researchers. In (Hadera et al., 2019), the authors proposed the use of Mean Value Cross Decomposition, and they tested their approach in pulping and steel production process. A game theory approach was proposed in (Leenders et al., 2019), where the Stackelberg game was applied to two literature-based case studies. A three-stage mixed-integer programming based decomposition strategy was proposed in (Zulkafli et al., 2020), showing that optimal or nearoptimal solutions can be obtained four times faster than with the integrated approach.

\subsection{Remarks}

The most extended approach used in the literature to deal with the optimal coordinated operation of cogeneration systems and process industries is the sequential one, where both systems are treated independently. When the scheduling of process systems under energy awareness is considered, if its main dynamics are close to the frequency electricity prices change, they must be considered explicitly in the production scheduling problem. Thus, when decisions regarding to electricity exchange are taken well in advance, top-down approaches are the most recommended strategy. Otherwise, bottom-up techniques are more suitable.

In many situations, when the sequential approach is considered, the results obtained can be suboptimal. It may be the case where even both processes cannot be treated independently due to the energy coupling existent between them. While the integrated approach is able to consider both systems at the same time, until now, the incorporation of the industrial process dynamics has 
not been studied for this problem, probably due to the great computational cost that it supposes. Furthermore, it is surprising that despite the need to comply with the legislation concerning the efficient operation of cogeneration systems, only a few papers consider this topic, being already outdated.

In this thesis we propose a new methodology based on the integrated approach, where the optimal operation, complying with the current legislation, of industrial processes and cogeneration units are considered simultaneously, being the dynamics of the first one explicitly considered. In this context, this has been possible thanks to the concept of time-bridging models, which will be used and adapted to obtain a dynamic optimization problem tractable from a computational point of view. Thus, we avoid the necessity of dealing with the coordination of two different optimization problems (iterative approach), while the most important features of the system are preserved. 


\section{Chapter 3}

\section{Dynamic-Integrated Scheduling approach}

In the previous chapters, first, we showed the benefits that provides the operation of industrial processes working with on-site cogeneration systems under price-based demand response programs. Then, we reviewed the state of the art regarding the optimal operation of such systems to fully exploit the possibilities given by these programs. There, we saw that to obtain the best results, the integrated approach, which considers the whole system at the same time, was the best option in many cases. Nevertheless, despite of the importance of considering the industrial process dynamic features in such optimization problem, we have not found any research focused on its explicit incorporation.

In this chapter we present a methodology to apply the integrated approach in a systematic way to systems where the main process dynamics cannot be neglected. Thus, first, we set the assumptions taken to develop such methodology. Later, we have divided the presentation into three different sections, one clearly focused on the system modeling, another one on the optimization problem formulation, and the last one on the industrial roll-out. Finally, we give some remarks, and we describe the methodology limitations and possible extensions. 


\subsection{Introduction}

In this work we are focused on those price-based demand response programs where the electricity price is given well in advance (Day-Ahead and TOU tariffs). For such programs, to operate in the Spanish electricity market, the electricity commitment must be communicated to the market operator before noon of the day before the price is negotiated and cannot be modified without incurring in penalties. In this work, we take some ideas from the top-down approach presented in Section 2.1 and we apply them to the integrated technique. Thus, we propose a dynamic scheduling approach in a moving-window fashion to compute the best operation strategy of the process-CHP system, given the electricity price, demand forecast, and raw material input for a predefined prediction horizon. The results implementation is carried out by the control system, which is responsible for the correct tracking of the set-points given by the optimization. The architecture of the solution proposed is shown in Figure 3-1.

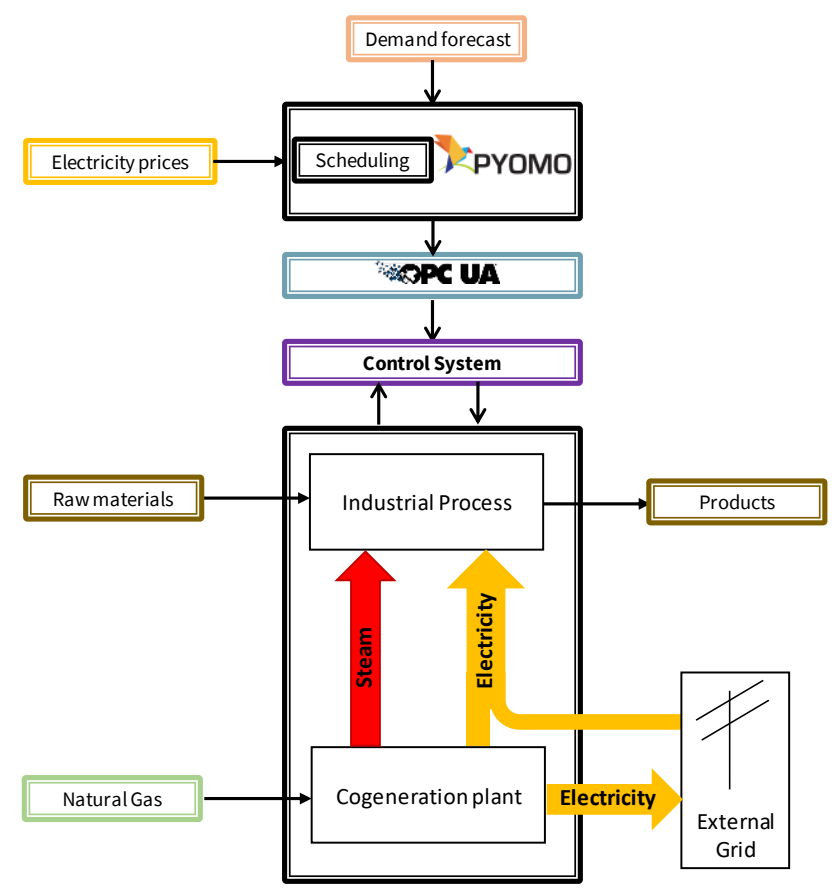

Figure 3-1. Architecture of the process optimizer interaction. 
To apply the methodology, consider now an industrial process that produces a single product in one production line, and whose dominant dynamics are close to the frequency of changes in electricity prices. Product storage is assumed not to be a problem, considering space, quality and security issues. Furthermore, the process has access to a CHP plant where heat and power generation are strongly coupled, while, in the integrated system (process $+\mathrm{CHP}$ ), only continuous operating decisions can be taken. Electricity can be imported and exported from the external grid through a retailer, and the current legislation related to the efficient generation in CHP systems must be met.

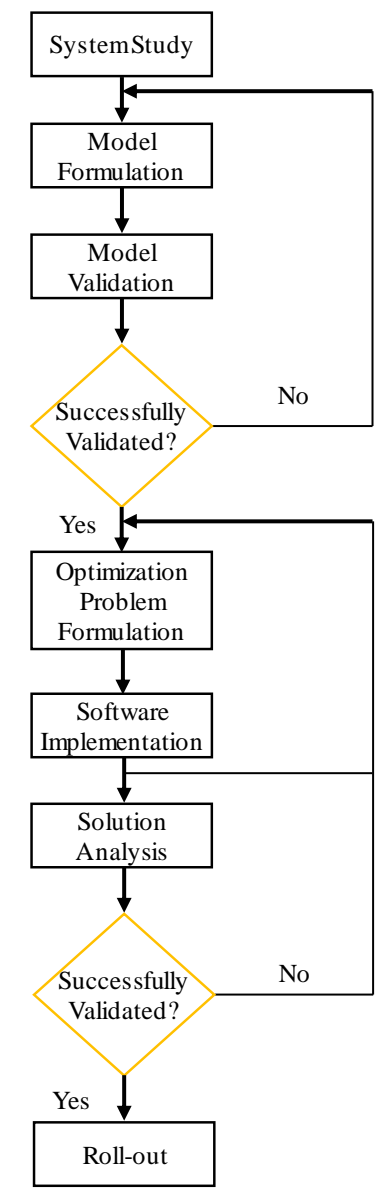

Figure 3-2. Methodology Scheme. 
The methodology consists of a series of stages that begin with a study of the system considered. A scheme of the proposed workflow can be found in Figure 3-2. Each system is different in many ways, so a good understanding of the process itself and its relationship with the utility system is key to an effective optimization problem design. In this study, the optimization aim, the decision variables, and the operational constraints must be clearly defined. Thus, knowledge about the functioning of the local electricity market and legislation concerning the efficient operation of cogeneration systems takes on special relevance.

\subsection{Model formulation and validation}

To model the integrated system, we recall the Time-Scale Bridging models, presented in Chapter 2 as an alternative to the inclusion of the control system dynamics in the production scheduling problem. There, a closed-loop model that shows the dependency of the process outputs with respect to the inputs was used instead of the rigorous plant dynamic model. Now, when we consider the implementation of the industrial process dynamics in the integrated approach, we have a very close problem. Such models can be understood as gray-box representations, where first-principles equations, like mass and energy balances, are merged with empirical approximations from plant data (Pitarch et al., 2019). In this modeling framework, the amount of first-principles or empirical equations must be chosen accordingly to the system complexity and the data available from measurements:

$$
\begin{gathered}
\frac{d x}{d t}=f\left(x(t), u(t), z(t), \theta_{F P}\right) \\
h\left(x(t), u(t), z(t), \theta_{F P}\right)=0 \\
\frac{d x}{d t}=r\left(x(t), u(t), z(t), \theta_{E X}\right) \\
s\left(x(t), u(t), z(t), \theta_{E X}\right)=0
\end{gathered}
$$

In Equation 3.1, we can distinguish between the first-principles equations of the model (3.1a), and its empirical equations (3.1b). Here, $x \in \mathbb{R}^{n}$ is the state 
vector , $u \in \mathbb{R}^{m}$ are known process inputs (manipulated variables or measured disturbances taking arbitrary values independently of the rest of the variables), $z \in \mathbb{R}^{q}$ are algebraic variables, $\theta_{F P}$ are the first-principles model parameters, $\theta_{E X}$ are the empirical equation parameters, both assumed to be constant, where $\theta_{F P} \cup \theta_{E X}=\theta \in R^{\rho}$, and $f(\cdot) \in R^{j}, h(\cdot) \in \mathbb{R}^{l}, r(\cdot) \in R^{i}, s(\cdot) \in R^{h}$ can be nonlinear functions of their arguments.

To obtain the model, the first step of this methodology consists in the establishment of the model inputs $(u(t))$ and outputs $(y(t))$. Previously, we must have defined the decision variables of the optimization problem, which can help us to define the model inputs, the optimization aim, and operational constraints, which will guide us to select the model outputs. As a guide, it is useful to consider the electricity, heat, and fuel consumption of the plant, the variables needed to compute the legislation indexes, and some other critical process variables for a correct plant operation.

The next step consists in searching relations between the inputs and outputs selected. Here, it may be useful to disaggregate the process into smaller pieces (control volumes). To do so, it is recommended to count with the help of a process expert. With the division made, we must use first-principles equations to describe those parts of the system where more details about the physical phenomena are needed (Equations (3.1a)). Now, let us assume that by using only first-principles equations the model is undetermined, meaning that the outputs cannot be completely computed using the inputs $u$. Therefore, the model has more degrees of freedom than the real system. Formally, this means that there are $\mathrm{n}+\mathrm{q}-\mathrm{j}-\mathrm{l}-\mathrm{l}-\mathrm{h}-\mathrm{m}>0$ free variables $\left(x_{E X} \subset x, z_{E X} \subset z\right)$, which must be a function of other variables representing the not well-known parts of the process. Therefore, some additional equations need to be identified from process experimental data (Equations (3.1b)).

To do so, let us assume that enough measurements are available in the process plant. Thus, the most usual approach is to set up a certain structure for the empirical equations that depends on some parameters $\theta_{E X}$, and obtain them using identification techniques (Isermann and Münchhof, 2010; Ljung, 1999). To do so, we recommend the use of a specialized software, like the System Identification Toolbox for MATLAB ${ }^{\circledR}$ (Ljung, 1988). In this methodology, we propose to carry out this step independently for each empirical equation. First, it is convenient to perform some experiments to check the correlation between 
the experimental inputs and outputs. Ideally, to reduce the computational cost as much as possible, linear empirical relations are desired. If that is the case, we propose the use of the state-space representation (Isermann and Münchhof, 2010), which can deal with Multiple-Input Multiple-Output (MIMO) systems, delays can be easily incorporated, and its structure is very convenient for the optimization problem formulation. Otherwise, if a linear structure is not possible, other approaches such as Hammerstein-Wiener models (Wills et al., 2013), symbolic regression (Cozad and Sahinidis, 2018), Neural Networks, or Fuzzy models (Nelles, 2013) could be used.

Once the empirical equations are found, let us recall that the first-principles equations incorporate another set $\theta_{F P}$ of unknown parameters. To find the value of such parameters, we propose to solve a least-squares (LS) constrained regression with $\mathrm{N}$ data samples collected at time instants $t_{1}, t_{2}, \ldots t_{N}$ (Tan and Li, 2002), where the model initial condition $x_{0}$ is also left as a decision variable:

$$
\begin{gathered}
\min _{\theta_{F P}, x_{0}} \sum_{t=t_{1}}^{t_{N}}\left\|\frac{(\hat{y}(t)-y(t))}{\sigma}\right\|_{2}^{2} \\
\text { s.t.: } \frac{d x}{d t}=f\left(x(t), u(t), z(t), \theta_{F P}\right), \quad x(0)=x_{0} \\
\frac{d x}{d t}=r\left(x(t), u(t), z(t), \theta_{E X}\right) \\
h\left(x(t), u(t), z(t), \theta_{F P}\right)=0 \\
s\left(x(t), u(t), z(t), \theta_{E X}\right)=0
\end{gathered}
$$

Note the reader that depending on the complexity of the set of Equations (3.1b), we could formulate a "centralized" approach where the first-principles and empirical parameters were searched at the same time, skipping one step in the methodology. Note however, that considering the possible nonlinear nature of the total set of Equations (3.1), if the set of total parameters is large enough, the resulting optimization problem could be very hard to solve, so this approach must be considered carefully.

Nevertheless, a very common setback for the presented approach is that, usually, in process industry, some of the measurements needed may not be 
available. To solve this problem, we propose a two-step methodology similar to the one presented in (de Prada et al., 2018):

- First, run a dynamic data reconciliation problem (Leibman et al., 1992) and obtain an estimation of all the variables and parameters needed to complete the set of first-principles equations (3.1a).

- Second, with a credible set of data, a regression stage is carried out. This stage can be performed using the Matlab ${ }^{\circledR}$ system identification toolbox, or if the structure of the resulting model is completely unknown, techniques like SOS constrained regression (Pitarch et al., 2019) or the approach presented by ALAMO (Cozad et al., 2015).

With this approach, we must be aware that the model obtained is not perfect, and it cannot be used to describe the internal physics of the system. However, although the values obtained may not be completely precise, they are coherent with the physics knowledge and data available, so it should be enough for optimization purposes.

Once the model has been obtained and parametrized, the next step is validation. Here, the model outputs are directly compared to the real system response. This is known as quantitative validation. In case that not enough data is available for this purpose, a qualitative verification can also be performed with the help of a process expert, where the transient response and robustness of the model is checked for different scenarios. In any case, the model needs to be simulated for this step. To do so, a symbolic manipulation of the gray-box model must be performed. We recommend the use of an acausal specialized software, like Ecosimpro ${ }^{\circledast}$ (Empresarios Agrupados, 2020), as a modeling and simulation environment. With this tool we can perform tasks like a degree of freedom analysis, handle algebraic loops, and check the existence of high-index problems. Note the reader that before running the simulation, we need a good initialization point. A good approach to obtain it is to add the initial condition as an adjustable parameter of the data reconciliation problem.

To sum up, we list the methodology steps previously described:

1. Set the model inputs and outputs.

2. Propose a set of first-principles equations where more information about the process physics is needed. 
3. Complete the model with empirical equations.

a) If enough measures are available, first, identify the black-box parts of the model and, later, perform a parameter estimation to obtain the first-principles equations parameters.

b) If not enough measures are available, first, estimate the variables and parameters needed running a dynamic data reconciliation problem, and later, perform a regression step to find the black-box model equations.

4. Validate the model obtained.

\subsection{Optimization problem formulation}

Once the model has been successfully validated, a dynamic-optimization problem in the general form (3.3) is to be formulated.

$$
\begin{gathered}
\min _{u(t)} J:=\int_{t_{0}}^{t_{f}} L(x(t), z(t), u(t), p) d t+M\left(x\left(t_{0}\right), z\left(t_{0}\right), x\left(t_{f}\right), z\left(t_{f}\right)\right) \\
\text { s.t. } f(\dot{x}(t), x(t), u(t), z(t), \theta)=0, \quad x\left(t_{0}\right)=x_{0} \\
h(x(t), u(t), z(t), \theta)=0 \\
g(x(t), u(t), z(t)) \leq 0 \\
x \in X, \quad u \in \mathcal{U}, \quad z \in \mathcal{Z}
\end{gathered}
$$

The Dynamic Algebraic system of Equations (DAE) that model the behavior of the plant $(3.3 b)-(3.3 c)$ together with the initial condition define an InitialValue Problem (IVP) which is solved from time $t_{0}$ to $t_{f}$. The way to obtain such model was illustrated in the previous section. In real systems it is common to find discontinuities that need to be modeled, nevertheless, it is well known that they must be avoided when using gradient-based solvers. If that is the case, a reformulation is needed, and one way to deal with this problem is the use of complementarity constraints. In (Biegler, 2010), four different formulations for 
the complementarity conditions were compared, and whether they should be implemented as constraints or as additional terms in the objective function was discussed. The results showed that the best way to deal with them is using a penalty term in the objective function and solving the problem for a penalty parameter. The IVP is also subject to additional inequality constraints $g(\cdot)$ like the electricity market interactions and legislation limits. While the main process and CHP model depend on each case study, the electricity market and legislation constraints can be applied to any other industrial plant with a cogeneration facility using the same optimization approach. Other inequality constraints may be bounds imposed to the decision and output variables.

The objective function (3.3a) includes the usual Lagrange (running cost) and Mayer (endpoint cost) terms, defined by functions $L$ and $M$ respectively. Typical expressions inside the Lagrange term may include the minimization of the operating costs, the maximization of the difference between the incomes and costs (profit), or the minimization of the cost per unit of product (specific cost). Therefore, for the problem considered, the cost related to heat and electricity generation, and the inputs obtained by the product and energy sales must appear somehow in this expression. In many occasions, when dynamic optimization problems are considered, the incorporation of additional terms to the objective function is necessary to obtain realistic results. For example, due to numerical problems, it is common to obtain highly oscillating solutions for the decision variables. Usually, to compensate this behavior, a term that penalizes such fluctuations is added to the cost function. Other example is the turnpike property (Dorfman et al., 1958; Ellis et al., 2014; Faulwasser et al., 2017), referred to the irregular response that appears at the final moments of the control horizon in many finite-horizon dynamic optimization problems. This behavior appears when the prediction horizon is large enough, so the optimizer "pays" the cost of the turnpike (the optimum steady-state) to reach the final point with the least possible cost. If the operation continues after the control horizon, this behavior is undesired, and one strategy to deal with this problem is the use of a penalty term in the cost function in the form of the Mayer term.

As was mentioned before, a common approach to deal with the complementarity constraints is to multiply the complementarity terms by adjustable penalty weights used to calibrate the influence that such penalties have over the cost function, whose value will depend on each case study. 
Although the tune of these parameters is key to obtain proper solutions, usually the methodology followed is not discussed in many works. Here, we present the strategy that has been used in this thesis:

1) Select some representative scenarios that cover the full operation spectrum in one day. It is recommended to have at least $2^{n}$ scenarios, where $n$ is the number of exogeneous input variables, understood as those whose value is determined by factors or variables outside the casual system under study. Typical examples are the electricity, or the raw material prices.

2) Find the minimum penalty value that affects the solution. For such task, the iterative bisection method can be recalled for each scenario with all penalty values set to 0 , except the one being tuned of course. To evaluate if the solution is affected or not, look if values for some relevant output variables remain constant between iterations or not. The minimum penalty value will be the supreme of the values found for all scenarios.

3) Afterwards, such minimum value can be increased ad-hoc to cope with the designer preferences. Note that, for the complementarity penalties, an increment from the above minimum values may cause distortions in the problem geometry without potential benefits, so we strongly recommend keeping such values to the minimum found.

When a penalty nominal value is found, a local sensitivity test is performed to check if small variations around such value have important effects on the optimization results. To do so, we followed this procedure:

a) Numerically evaluate the optimization results for each of the previously selected scenarios using the nominal penalty value.

b) Set a $1 \%$ increment w.r.t the candidate value and evaluate numerically the solution again for each scenario.

c) Compute the sensitivity as the percentage ratio of the output variation computed for each scenario w.r.t the corresponding nominal value obtained in step a). If the highest sensitivity obtained for all scenarios is small enough (e.g., <0.1\%), the optimization with the proposed penalty value appears to be locally robust. Otherwise, a new candidate must be found by alternative methods. 
With this methodology, a trade-off between robustness and computational time is found for the tunning of the penalty weights. However, if a more robust tunning is desired, the mentioned scenarios can be replaced by others generated following a Monte Carlo approach, for example. However, the number of scenarios is expected to increase, and therefore, the number of experiments that must be run each time the penalty weights are tuned.

In the literature we can find a wide variety of methods to solve the infinitedimensional problem posed in (3.3). In (Biegler, 2010), we find that when the size of the problem increases, optimality conditions become increasingly harder to apply and, therefore, efficient numerical methods are required. Such methods can be classified as shown in Figure 3-3:

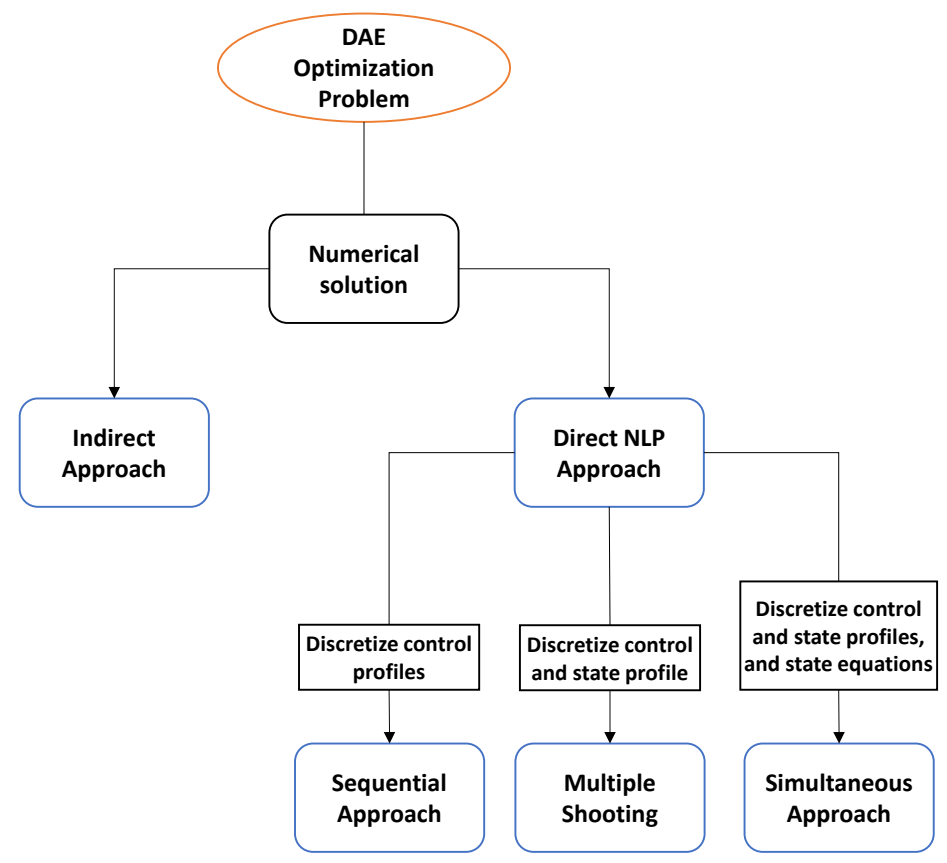

Figure 3-3. Summary of the numerical methods most used to solve the DAE optimization problem.

The indirect approach is based on the idea of first optimize and then discretize. First, the solution of the first order necessary conditions for optimality are obtained using Pontryagin's Maximum Principle (Pontryagin et al., 1962). Thus, for problems without inequality constraints, the optimality conditions can be reformulated as a set of differential-algebraic equations, which must be 
solved. The main advantage of this method is the fact that it reflects the theoretical solution of the infinite-dimensional problem, however, one important problem are the boundary conditions. Often the state variables are given as initial conditions and the adjoint variables as final conditions. This leads to a Boundary Value Problem (BVP) that can be solved with different approaches, including single shooting, invariant embedding, multiple shooting, or discretization methods such as collocation on finite elements. A review of these approaches can be found in (Cervantes and Biegler, 2000). Therefore, the difficulties associated when solving the BVP problem and the need of handling inequality constraints motivate the formulation of constrained, finitedimensional problems that can use the full machinery of large-scale NLP solvers.

Unlike the indirect approach, when the direct approach is considered, the dynamic optimization problem is first discretized and then solved. If the discretization is not carried out properly, it may lead to a problem when path constrained, and singular problems are considered. Depending on the discretization level, we can find three different methods to solve this problem: the sequential approach, multiple shooting, and the simultaneous approach.

In the sequential method, or single-shooting approach, only the control profiles are discretized, which are represented as piecewise polynomial functions. The problem is solved in an iterative fashion in three sequential steps (see Figure 3-4). First, an NLP optimization problem is solved where the optimum control profile is found for some initial conditions. Then, the solution is sent to a DAE solver where the state profiles are computed, and the objective function is evaluated. To close the loop, the gradients of the objective function and constraints with respect to the input variables are computed and, finally, they are sent to the optimizer along with the objective function value and a new iteration starts. Advantages of this approach are the fact that it relies on efficient DAE solvers and the optimization problem will be relatively small. However, the required repeated numerical integrations of the DAE solver may lead to high computational cost. Furthermore, a failure in the solution is fatal for the optimization loop, so unstable systems must be avoided. Finally, path constraints are difficult to evaluate and usually they must be handled approximately. 


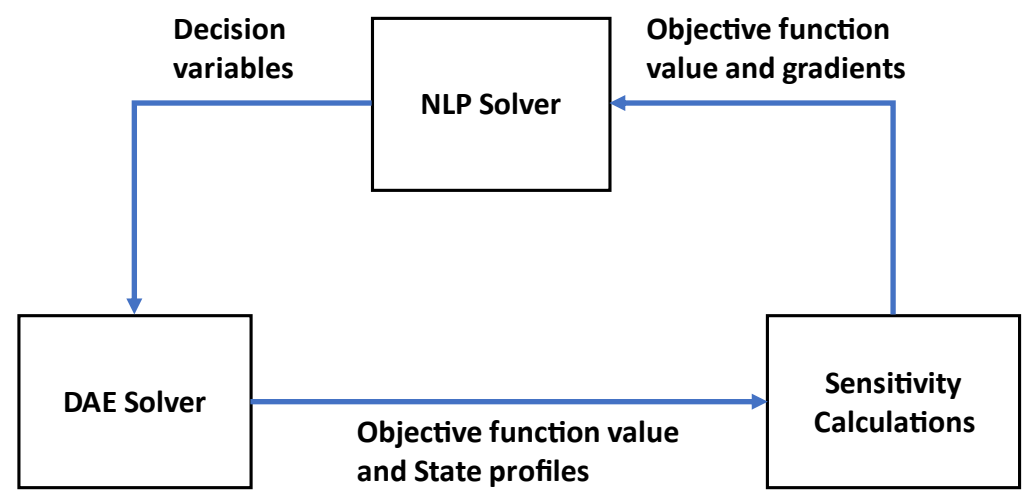

Figure 3-4. Sketch of the sequential dynamic optimization strategy (Biegler, 2010).

In the multiple shooting approach, both the control vector and the state profile are discretized. It is an evolution of the sequential method that emerges to deal with unstable DAE systems. The solution approach is the same as the one presented before, but now; the time domain is divided into independent elements that are bounded by equality constraints between the adjoint elements of the discretization grid to ensure continuity of the states over time. Control variables are treated in the same manner as in the previous approach and the initial conditions of each element are included as decision variables. As main advantage of this method we find that now unstable and ill-conditioned systems can be considered. Furthermore, exploiting the problem structure, the simulation stage can be accelerated with parallel programming techniques, and path constraints can be easier implemented in the discretized points, although they cannot be assured between elements. On the contrary, the optimization problem complexity increases, the number of iterations may not be reduced, and more sensitivity information is needed, since gradients are needed for both the control variables and the initial conditions in each element.

The simultaneous approach deals with a full discretization of the optimization problem, discretizing the state and control profiles along with the state equations. Typically, such discretization is performed using orthogonal collocation. Thus, a large-scale NLP problem is obtained allowing a great deal of sparsity and structure, that can be exploited by modern NLP solvers. Furthermore, path constraints can be easily implemented, and difficult problems can be treated, including unstable and singular control systems. Additionally, the 
approach is fully simultaneous, so a DAE solver is no longer needed. This avoids convergence problems and allows that sensitivities can be directly evaluated exploiting automatic differentiation techniques (Tolsma and Barton, 1998). However, the adaptive discretization is lost, and care must be taken when selecting the number of finite elements and their length.

Given the difficulties that may be found to solve the two-point boundary problem proposed by the indirect methods and to treat inequality constraints, we think that to deal with the problem considered, direct methods are the best alternative. Among them, when the sequential approach is considered, the use of a DAE solver provides accurate time evolutions of the state. However, depending on the model size, the computational cost for simulating in each iteration of the optimizer can be unacceptable. Hence, this approach is adequate when the accuracy of the solution is key. Multiple shooting becomes interesting when the system stability is a problem and sufficiently high-quality solutions of the state profiles are desired. However, most of the disadvantages of the sequential approach may be applied. Finally, with the simultaneous approach the whole problem is discretized, and while the solution may not be so accurate and the size of the optimization problem increases, the many calls to a numerical simulator are avoided and much lower computational times are reached. Since computational time is important for our purposes in this application, we decided to follow this last approach. The optimization problem (3.3) can thus be coded in any modern programming language for numerical optimization such as Pyomo (Hart et al., 2017, 2011), CasADi (Andersson et al., 2018), or GEKKO (Beal et al., 2018).

For an optimization problem, the knowledge of the initial conditions is key in order to obtain good solutions. However, in the problem considered, the optimization problem must be executed several hours before the results are implemented. This feature makes the estimation of the initial state necessary. The easiest way to do it is running a simulation with the expected inputs, from the instant the optimization is launched, until the moment the results are implemented. In this context, counting with a digital twin could be extremely useful, although the very same optimization model could be used instead. Of course, the later the optimization is run, less uncertainty will be found, but still some unexpected events may occur between the optimization run and the results implementation. To minimize the effect of this problem, an alternative 
optimization problem, where the electricity commitment is fixed, should be launched as close as possible to the implementation moment.

In order to validate the results obtained from the optimization, first, a simulation test for several representative operation days is performed. Here, the optimization is launched several times with different electricity prices in a preselected operating scenario, and the optimization results are validated by experts. Special attention must be put on the results obtained at the end of the day, since the turnpike property may cause some problems (Faulwasser et al., 2017). If the one-day validation is positive and the optimizer provides acceptable solutions, a long-term validation should be performed, so the optimizer is tested for several days or even months, checking the transition results between different days and operating scenarios.

\subsection{Industrial Roll-out Concept}

Once the optimization tool is successfully validated, it is ready for implementation. In this section we propose a methodology for the implementation and use of such tool in an industrial environment. Since we have worked with a simulated factory, we understand that different problems, apart from the ones that we have faced, will arise in real environments, so we present this methodology as a concept. We have worked in this task because we are aware that this topic is usually neglected in the literature, and we consider that it is key in order to decide whether an optimization tool is ultimately useful or not. We propose an architecture with three different modules, as shown in Figure 3-5.

\section{Optimization module}

The objective of the optimization tool, developed using the methodology shown in the previous sections, is to compute the electricity that must be dealt with the market considering an operation strategy that can be refined later. The tool acts as a server which once per day receives the value of exogeneous variables, like the electricity price or the demand forecast, along with the expected initial state from the visualization module, and computes the optimal operation strategy, together with the electricity commitment, which are sent in return.

Given that the optimal strategy computed by the optimizer is obtained several hours before its implementation, it cannot be applied directly in the 
industrial process without refining. That is why we propose to separate the problem into two different phases. In the first phase, carried out by the optimizer, the optimal commitment is computed for an expected industrial process operation. Thus, the electricity exchange with the market is fixed, and it is set as a hard constraint in the second phase carried out by the MPC module. Therefore, the objective of the MPC is to compute the optimal operation strategy while meeting the commitment previously fixed. The formulation and implementation of the MPC, which is out of the scope of this work, will be similar to the one presented for the optimization tool, but with more emphasis on control aspects instead of energy.

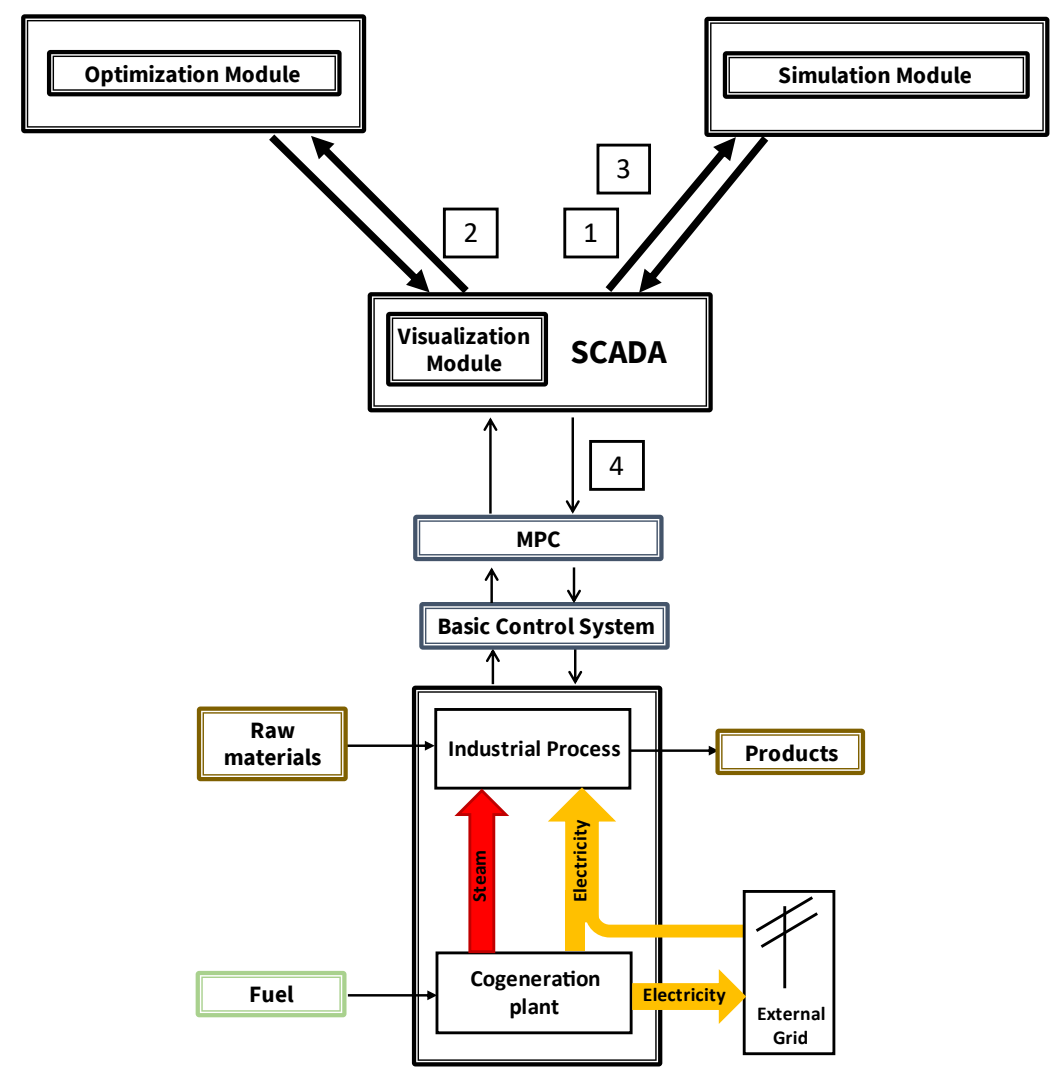

Figure 3-5. Roll-out architecture concept. 


\section{Simulation module}

It is a simulation that represents closely the real process behavior, maintaining a limited computational cost. Depending on the objective pursued, it can be from the model used for optimization, to a much more complex model representing with more detail the desired parts of a process system. The main use of this model is to estimate the optimization initial state. It acts as a server which receives an operation strategy and computes the expected response. If such strategy is the one obtained from the optimization, it can be used to analyze its consequences before carrying out its implementation in the real system (what-if analysis).

\section{Visualization module}

A common problem when it comes to the implementation of optimization algorithms in the process industry, is the lack of programming skills by the end user. Furthermore, it does not help how unintuitive the programming environments usually are. That is why we proposed the development of a visualization module integrated with the process SCADA in order to make the experience as user-friendly as possible. This module acts as the core of the proposed architecture, it is a client from where optimizations and simulations can be run and configured. Therefore, it is communicated with all the other modules, and the process control system.

\section{Operation steps}

Next, we explain the steps that should be followed to work with the proposed system in a daily use. Such steps have been remarked with numbers in boxes in Figure 3-5.

1. Each day, before the day-ahead electricity market stops receiving bids, the simulation module must be executed from the SCADA to compute the expected response until midnight, so the optimization initial state is obtained. Thus, the current state of the process is set as the simulation initial state and the operation strategy expected for the rest of the day until midnight as input.

2. From the SCADA system, the computed initial state, along with the expected exogeneous variables for the next day are sent to the 
optimizer, and the optimal operation strategy and electricity commitment is computed.

3. The optimization results must be evaluated. To do so, we can send them to the simulation module and analyze the implications that its approval would have in other parts of the process. Then, a disjunction occurs:

a. If the results are satisfactory, the electricity commitment is stored in the SCADA and sent to the market.

b. Otherwise, step 2 can be repeated until a valid solution is obtained.

4. From midnight, the commitment results are sent to the MPC system as hard constraints where the optimal operation strategy is computed. The results are implemented in the industrial process using the basic control system.

\subsection{Remarks}

In this chapter, we have presented a methodology to include industrial process dynamic features in the integrated scheduling approach for the optimal operation of industrial processes working with cogeneration systems. The methodology here proposed is valid for the assumptions indicated at the beginning of this chapter. The points referred to the process dynamics compared to the electricity prices variations are especially relevant. As relevant as the straightforward aspect of considering CHP systems where heat and power generation are strongly coupled indeed. Note that, if the main process dynamics are much faster than the changes in the electricity price, they can be neglected, and the optimization problem would be much easier to formulate and solve. Furthermore, if no coupling exists in the cogeneration process, its operation can be split from the industrial process, and a sequential or iterative approach could be taken. Of course, the proposed approach (as other DR programs as well) may not be effective in large plants where the electricity price fluctuations have a low impact on the plant economics, either because a lack of data on the actual power consumption of the equipment inside individual plants, or because the contribution of the electricity price is small compared to raw-material costs and product revenues. 
On the other hand, if discrete operating decisions need to be considered during normal operation, the presented approach is not directly applicable (unless some suitable reformulation/approximation of these can be done), and the optimization problem must be reformulated as a dynamic MINLP problem. This would be the case if different products could be produced in the industrial plant in one or more operating lines, or the CHP system had the possibility of turning on or off some equipment. The efficient formulation of such problems is also a relevant research problem nowadays, so future work could be oriented in this direction. Furthermore, uncertainty in diverse aspects like the electricity price, demand forecast, or raw material reception could also be considered in the formulation. This way, the dynamic optimization problem would have to be solved using a robust or stochastic optimization approach, and an efficient formulation for such aim could be studied. 



\section{Chapter 4}

\section{Case Study Framework}

From this chapter on, we will apply the methodology proposed in Section 3.2 to a simulated sugar factory working with a cogeneration unit. As we showed in Section 1.1.2, the sugar industry may take advantage of the possibilities presented by the price-based Demand Response programs in order to be more competitive in a very saturated market. Thus, this part of the thesis must serve as a proof of concept of our methodology, allowing us to test it in a safe environment without security and money issues.

Therefore, in this chapter we apply the first part of such methodology, where the case study framework must be analyzed. First, we give a general description of the simulated process considered as case study without going into detail, considering that more information will be given in the next sections if necessary. Then, we introduce the operation and management of the different electricity markets in Spain, paying special attention to the Day-Ahead one. Later, we review the most important aspects of the legislation applied to the efficient operation of cogeneration plants. And finally, we conclude by analyzing the system from an optimization perspective, defining the optimization aim, decision variables, and operational constraints derived from the study of the other subsections. 


\subsection{Process description}

As case study, we have considered a simulated beet-sugar factory that would be placed in the north of Spain. Here, the sugar extraction is a seasonal business carried out approximately from October to February. One feature of the sugar industry is that it can be classified as a hybrid process, meaning that it is composed of a continuous part (Beet-end) and a discontinuous one (Sugar-end). This has been represented in Figure 4-1. In this scheme, we can also see that to obtain sugar, besides of beet, other inputs like water, lime, or $\mathrm{CO}_{2}$ are required. Furthermore, some by-products which can be used for secondary purposes are obtained like pulp, molasses, and electricity.

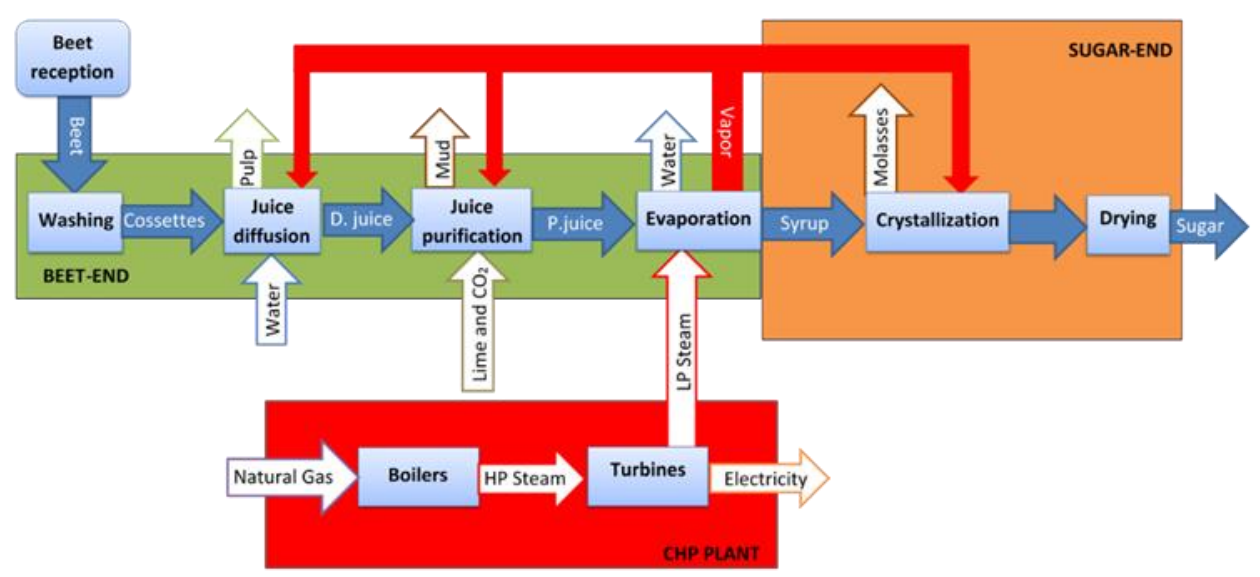

Figure 4-1. Scheme of beet-sugar factory considered.

In such figure, the different process stages can also be observed. In the beet reception, the received beet is stored in big piles outside the factory. The time the beet spends there is a crucial variable, considering that due to diverse problems as rotting or frosts, much of the raw material can be lost if this waiting time is extended too much. Therefore, long storage times should be avoided a priori. However, beet harvesting can be delayed because of rain, so this uncertainty, in many cases, means that industry managers prefer to increase the storage time to dissipate the possibility of running out of raw material. The importance of such problems varies sharply with the weather and, therefore, with the place where the factory is situated. In our case, the north of Spain is particularly cold during winter so they cannot be neglected and, ideally, the less time beet spends stocked, the better. For the considered factory, the beet 
processing rate must be established between $8880 \mathrm{t} / \mathrm{d}(370 \mathrm{t} / \mathrm{h})$ and $10320 \mathrm{t} / \mathrm{d}$ $(430 \mathrm{t} / \mathrm{h})$, considering that reaching a new steady state after changes in the beet rate takes around 5 hours.

Before the diffusion process takes place, beet must be washed and cut into thin slices called cossettes. This way, the sugar extraction is maximized. In the diffusion stage, the beet is put in contact with hot water in countercurrent, so the sugar inside is extracted by mass transfer. As main product of this process, the diffusion juice is obtained, which is sent to the purification stage to continue the sugar extraction process. Besides, exhausted pulp is obtained as byproduct. Given that the pulp still has a big amount of sugar and water inside, it is sent to a press process in order to extract as much water as possible, which is later recirculated to the diffusion. Finally, the pressed pulp is dried and can be sold as cattle feed.

After diffusion takes place, besides of sugar, many other impurities are removed from the beet. Unlike sugar cane, the impurities extracted are not desirable, so they must be removed in a purification stage. In this part of the process, first, the diffusion juice is preheated using steam obtained from the evaporation stage and, next, it is treated with two purification stages in series where impurities are precipitated and filtrated. As a result, the purification juice is obtained.

Brix degrees is a unit used in the food and drink industry to measure the concentration of solids in a stream. Considering that after the purification almost all the solids are sugar, we can say that from this stage on, the brix degrees represent the sugar concentration in the juice. The aim of the evaporation section is to increase such concentration removing as much water as needed to reach a particular brix point for the output stream (syrup). In the evaporation section of the considered factory there are six different evaporation effects (groups of evaporators working at the same pressure) placed in concurrent. There, the less concentrated juice is put in contact with the highest-pressure steam obtained from the cogeneration plant. With this arrangement, the steam produced in the first effects serves as heat source for the later ones and other parts of the factory. Traditionally, sugar processes present an excellent energy integration taking advantage of the vapor obtained in the evaporation stage to fulfill the heat energy demanded by the rest of the process (Urbaniec, 1989). In the plant considered, part of the vapor obtained from the six evaporation effects 
is used to fulfill the steam demand that comes, mainly, from the diffusion, purification, and crystallization stages, being the last one the biggest consumer. This feature makes the pressure inside each evaporator a critical control variable and, hence, the pressure of the steam delivered to the evaporation from the cogeneration plant.

In the sugar-end, the syrup received from the evaporation stage is treated to obtain commercial sugar in a batch process. Essentially, this process is carried out throughout several crystallization and centrifugation stages, where the maximum quantity of sugar is crystallized, obtaining as a by-product an exhausted solution of water and sugar that cannot be further treated (molasses). The crystallization stage, which is essentially a slow evaporation process performed at vacuum conditions, is the biggest steam consumer of the evaporation section. Furthermore, crystallizers and centrifuges are important electricity consumers. Since it is a batch process, the steam and electricity demand are irregular, and consumption valleys or peaks may cause important problems in the rest of the process. Thus, the correct synchronization between the two parts of the process is key in order to optimize the process operation and avoid bottlenecks. This problem has been deeply studied in the literature, where the steadiest energy consumption is usually searched (Acebes et al., 2019; de Prada et al., 2019). More details about the sugar production process can be found in (Asadi, 2005; der Poel, 1998).

Regarding the cogeneration plant, in the factory considered the installed CHP plant counts with three different boilers and three backpressure turbines able to produce up to $11 \mathrm{MW}$ of power. A scheme of the cogeneration unit is presented in Figure 4-2. Natural gas is used in the boilers to obtain superheated High Pressure (HP) steam. The temperature of the steam obtained can be controlled using a heat exchanger (ATP), where it is put into contact with a manipulated flow of fresh water. Next, steam is expanded in the turbines or passed through a bypass valve if necessary, obtaining superheated Low pressure (LP) steam which must be saturated using water before sending it to the evaporation stage. The power generated in the turbines can be controlled manipulating the steam flow passing through them. During normal process operation, if all the steam obtained in boilers is used to obtain electricity, more power than the needed for the factory operation is generated. If the connection with the external grid is not considered, part of the steam needed by the process must be bypassed to avoid 
electricity surpluses. As it was mentioned before, controlling the pressure of the steam sent to the evaporation stage results key to a correct operation. In the case studied, a split range controller is used to control such pressure, manipulating the amount of steam that passes through the bypass and the relief valve placed before the saturator.

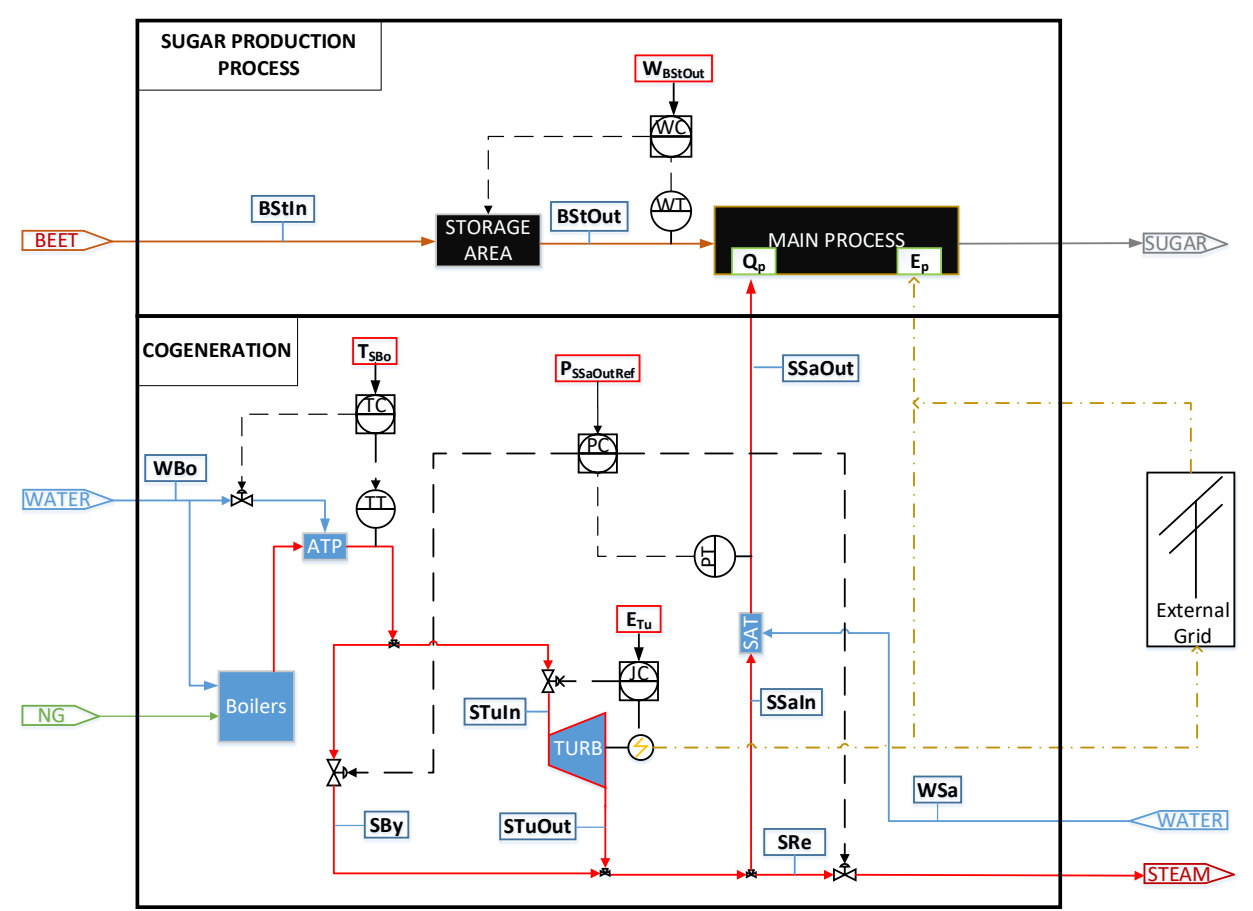

Figure 4-2. Simplified process flow diagram of the considered case study with the main existing instrumentation and power connection to the external grid.

\subsection{Electricity trade in Spain}

As mentioned in the previous section, the sugar plant considered in this work counts with a cogeneration system capable of filling the heat and electricity needs of the main process. However, sometimes, due to a wide variety of factors, the electricity generated in the cogeneration plant may differ from the one needed in the process. Thus, if the power generated is less than the one requested, it is necessary to buy electricity and, otherwise, it can be sold to obtain some revenue. Hence, to add this information into the optimization problem, it is necessary to know the main alternatives to trade electricity in Spain, which are described in this section. 
Spain participates in the Iberian electricity market (MIBEL), which is composed essentially by Spain and Portugal (OMIE, 2020). Furthermore, it can exchange electricity with other markets in Europe and Morocco, being the total amount of power that can be exchanged around the $3 \%$ of the total power installed (106.764 MW). The Iberian electricity market is managed by the Market Operator (MO) known as Operador del Mercado Ibérico de Energía (OMIE). This market, as in other countries, is formed by several markets that take place sequentially, where generators and consumers exchange power for different time horizons (See Figure 4-3).

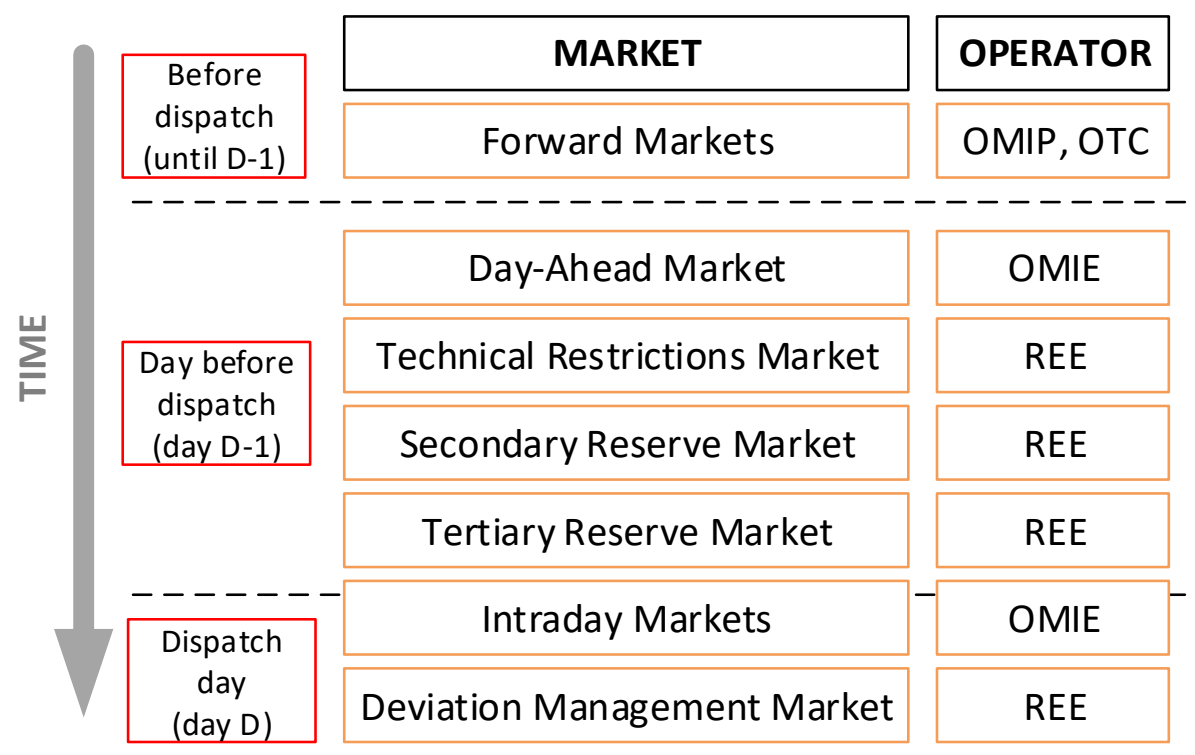

Figure 4-3. Electricity markets sequence.

First, the electricity forward markets take place weeks, months, or even years before the electricity exchange or the financial clearance between buyers and sellers occurs. They are key in order to let agents manage the risk inherent to new investments or operation. There are two different forward markets in Spain: the regulated-forward electricity market, managed by the Portuguese Market Operator (OMIP), and the non-regulated bilateral contracts market, also known as Over The Counter (OTC) (Energía y Sociedad, 2014a). 
Regarding the bilateral contracts, one can distinguish between physical contracts and financial contracts:

- In physical contracts, also known as Power Purchase Agreement (PPA), generator and consumer agents agree to exchange actual electricity for a determined time period with a fixed financial condition.

- In financial contracts, generators and consumers reach an agreement regarding the electricity price for a predefined amount of time. Therefore, they will participate in the Day-Ahead market, but the money they receive or pay will be the difference between the price negotiated and the clearance market price of each day.

The OMIE is in charge of two different markets, the Day-Ahead and the Intraday market. The main electricity market is the daily one, also known as DayAhead electricity market. Here, supply and demand bids are sent to the Market Operator until 10:00 AM of the day before the exchange takes place. At that time, after matching electricity supply and demand and, having considered different technical constraints, the electricity price is fixed for each hour of the next day.

The intraday electricity markets are designed to let the participants of the Day-Ahead one, revise their commitment and adjust their program to the needs they expect in real time. In Spain there are two different intraday markets: The auction market (operated by the OMIE) and the continuous European crossborder market. Regarding the intraday auction market, it is divided into 6 different sessions with different programming horizons that take place with the time distribution showed in Table 4-1, and each of them work in the same way as the Day-Ahead one. 
Table 4-1. Intraday market time distribution

\begin{tabular}{|c|c|c|c|c|c|c|}
\hline & Session 1 & Session 2 & Session 3 & Session 4 & Session 5 & Session 6 \\
\hline $\begin{array}{c}\text { Opening } \\
\text { time }\end{array}$ & $14: 00$ & $17: 00$ & $21: 00$ & $01: 00$ & $04: 00$ & $9: 00$ \\
\hline $\begin{array}{c}\text { Closing } \\
\text { time }\end{array}$ & $15: 00$ & $17: 50$ & $21: 50$ & $01: 50$ & $04: 50$ & $9: 50$ \\
\hline $\begin{array}{c}\text { Schedule } \\
\text { Horizon }\end{array}$ & $\begin{array}{c}24 \text { Hours } \\
(1-24 \\
D+1)\end{array}$ & $\begin{array}{c}28 \text { Hours } \\
(21-24 \\
\text { and 1-24 } \\
D+1)\end{array}$ & $\begin{array}{c}24 \text { Hours } \\
(1-24 \\
D+1)\end{array}$ & $\begin{array}{c}20 \text { Hours } \\
(5-24)\end{array}$ & $\begin{array}{c}17 \text { Hours } \\
(8-24)\end{array}$ & $\begin{array}{c}12 \text { Hours } \\
(13-24)\end{array}$ \\
\hline
\end{tabular}

The continuous intraday market was created by the European Union in 2018 to promote the power exchange between Member States and the use of renewable energies, increasing the overall system efficiency. One particularity of this market is that adjustments can be made up to one hour before the moment of delivery (OMIE, 2020).

Aside of the mentioned above, in order to ensure the balance between generation and electricity consumption with a good level of quality and continuous supply, generators and consumers offer some services to the system, organized in markets managed by the System Operator (SO) Red Eléctrica Española (REE) (REE, 2019). Such markets are known as the Ancillary Service Market, and they can be divided into three different groups: Technical Restrictions, Complementary Services, and Deviation Management (Lobato Miguélez et al., 2008).

The technical restrictions are solved on three different levels with a market mechanism: Restrictions related to the schedule obtained after the Day-Ahead market, others that arise after each intraday auction market, and the ones faced in real time.

The deviation management solves almost in real-time the deviations between generation and consumption. These markets take place after each intraday auction market. During normal operation, the generation agents send their deviation forecast to the System Operator, which are added to the variations expected in the renewable energy generation. In case that the forecast deviations exceed $300 \mathrm{MW}$ in hourly average, the System Operator will call the 
deviation management market to ask for offers from the generators in the opposite direction to the expected deviations (Energía y Sociedad, 2014b).

Regarding the complementary services, they can be divided into three different groups:

- Frequency-Power regulation is divided into three reserve services depending on the time horizon applied:

- Primary reserve: It is oriented to solve small imbalances, it is compulsory for every single generator, and there is not any market to manage it, so it is unpaid. It is formed by the speed regulators of the generators. It has a time horizon of 30 seconds.

- Secondary reserve: This market takes place after the Day-Ahead technique restrictions one. Its aim is to correct deviations in the frequency and, also, between the Spanish-French connection. If an imbalance continues during a certain amount of time, the secondary service will take over, releasing the primary resources to face new imbalances. This service is composed of a flexible reserve of energy that can be used when imbalances are important. The time horizon goes from 30 seconds to 15 minutes, and the dynamic response must be less than 100 seconds. This service is paid in a marginal way for two different concepts: Availability (Power band) and usage (Energy).

- Tertiary reserve: Its aim is to recover the reserves in case the secondary service must be used. This market takes place in the last hour of the day before the dispatch.

- Voltage control can be divided into two parts: one compulsory and not paid for all the service providers (big generators, qualified consumers, carriers and distribution operators), and another one voluntary and paid according to the regulation.

- Restoration service is provided by groups of generators able to start their operation in a determined amount of time without external power after a zero in the general voltage of the facility occurs and keep their generation stable while the supply is restored.

Due to the features of the process considered, in this work we are going to consider only the participation in the Day-Ahead electricity market. Therefore, a 
deeper explanation of the functioning of this market is given in the next subsection.

\subsubsection{Day-Ahead Market}

The Day-Ahead market is the most important electricity market in Spain. There, generators and consumers bid on a determined amount of electricity for each hour of the next day. Bids can be presented to the market operator until 10:00 AM. The selling or purchase offers (Energy and price) can be composed of 1 to 25 sections for each of the 24 hours of the day. Once the agents have presented their bids, the MO will aggregate them and form the offer and demand curve for each hour. The price is determined following a marginal procedure, where the final electricity price will be obtained by matching both curves. This means that regardless of the bid sent, every single seller receives the same amount of money for the electricity sold, and all the buyers must pay the same price (See Figure 4-4).

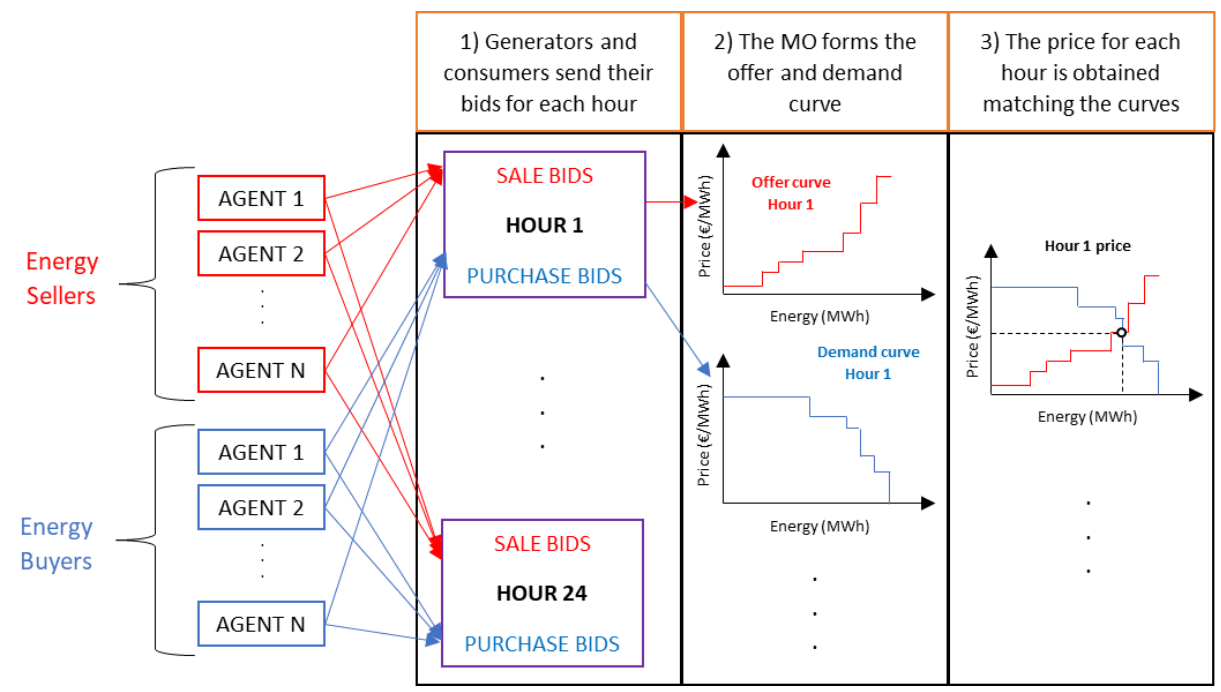

Figure 4-4. Day-Ahead Market description. Source: (Energía y Sociedad, 2014c).

It must be outlined that generators place their bids attending not only to the operating cost, but also to the opportunity cost. The opportunity cost is the income that generators are renouncing to, because of selling electricity in a certain moment. For example, hydropower companies can change their bid depending on the amount of water remaining in their reservoirs. If it was raining 
a lot, and reservoirs were almost full, they would be forced to release water, so their bid would be close to $0 € / M W h$. However, if reservoirs were at mid capacity, they could wait for another moment to sell the electricity at a higher value, so they would offer the electricity at a very high price. Other technologies, like renewable energies, usually bid their production at a price $0 € / \mathrm{MWh}$ because for them is very difficult to store energy (Energía y Sociedad, 2014c).

Aside from the mentioned above, it should be considered that generators can only make one offer for each physical unit they dispose of. Only for low power installations, they can present many physical units packed in one offer. Sometimes, the bids are accompanied by some complex conditions, such as the "minimum incomes constraint", which evaluates the generator incomes, and if they are less than a determined amount, all the bids are withdrawn (Gallego and Victoria, 2012).

Consumers are mainly composed of retailer companies and large industries. Retailers will bid on the electricity at the highest allowed value (180€/MWh), this way, they will ensure their participation in the bid and the obtention of the expected electricity demanded by their customers. Other consumers will try to purchase the electricity at a much lower value. As some of them are not forced to purchase it, they will only do it if the electricity price is convenient. Once all the bids are received and curves are obtained, an optimization algorithm is run (Euphemia) (NEMO Commitee, 2019), and the electricity prices for each hour are obtained matching the offer and demand curves.

\subsubsection{Price formation}

Only considering the Day-Ahead market, in Spain, there are essentially two ways for accessing such market: Directly as market trader, or through a retailer. Regarding the selling price, if generators go directly as traders to the market, the price will be the one fixed by the market minus some penalties that may be applied in case the production differs in excess from their commitment. If they participate through a retailer, the price obtained will be the market price minus a commission (the deviation penalty is assumed by the retailer). Other options where generators and retailers accord some price that can be fixed or segmented per hours may also exist. This way, generators look for covering from the risk of the market. 
In relation to the buying price, it is composed of an energy and a taxes term (Gallego and Victoria, 2012).

$$
P_{\text {Buy }}=P_{\text {Energy }}+P_{\text {Taxes }}
$$

The energy concept is the price paid for the electricity consumed. If the consumer participates directly in the market, the price paid for the electricity will be the one obtained in the market plus a penalty term regarding deviations from his consumption commitment. If he goes through a retailer, the deviation term will be assumed by the retailer. In this context, the price-based Demand Response programs appear as offered tariffs. The three most common tariffs are:

- $\quad$ Fixed price: The price is fixed and it is the same for every hour.

- Time Of Use (TOU): The price is fixed in hour segments. They may be composed of two or three segments.

- Real-Time Pricing: The price depends directly on the price established in the Day-Ahead market. It can be seen as the price of the market plus a commission.

With respect to the taxes term, it is mainly composed of the following concepts:

- Access tolls paid to the power distributor and carrier.

- VAT.

- Electrical tax fixed by the government.

- Meter rent.

\subsection{Legislation about cogeneration plants efficiency}

Directive 2012/27/UE (Official Journal of the European Union, 2012), is one of the tools used by the European Commission to achieve the objectives fixed towards 2050, related to energy efficiency and carbon emissions reduction. It establishes rules designed to remove barriers in the energy market and overcome market failures that hinder efficiency in the supply and use of energy. The requirements laid down in such Directive are minimum requirements for the Member States, which must transpose them adapting specific measures for each state. Among the different rules provided by the Directive, it establishes a 
method for measuring the efficiency of cogeneration plants and defines the concept of high-efficiency cogeneration.

In Spain, Directive 2012/27/UE was transposed by Royal Decree 56/2016 of February 12th, 2016, related to energy efficiency. It dedicates Chapter IV and, specifically, Article 13, to promote the efficiency in the production of energy used in heating and cooling. Furthermore, it modifies Royal Decree 616/2007 of May 11th, 2007, which provides a framework for promoting high-efficiency cogeneration in Spain and defines a method for calculating the Primary Energy Savings (PES) in its annex III. Law 24/2013 of December 2013 on the electricity sector, regulates electricity production from renewable sources, cogeneration, and waste. To encourage cogeneration, it establishes that high-efficiency cogeneration systems will benefit from a different remuneration system and priority in the dispatch, access, and connection to the grid.

Currently, Royal Decree 413/2014 of June 6th, 2014, establishes a framework based on the fundamental principle that the remuneration system must allow facilities of this type to cover the necessary costs to compete fairly with the other technologies available on the market, generating a reasonable return. Therefore, it defines that in addition to the remuneration obtained for the sale of electricity at the market price, the facilities will receive during the regulatory lifespan ( 25 years in the case of cogeneration plants) a specific payment comprising two additional compensatory components:

- Investment subsidy: Remuneration per unit of capacity to offset the cost of investment (reviewed every 3 years).

- Operating subsidy: Remuneration per unit of energy, which together with estimated operating income, meets the estimated operating costs (reviewed every 6 months). 
Methodology for determining the efficiency of cogeneration processes

To measure the efficiency of cogeneration processes, Directive 2012/27/UE defines in Annex II the Primary Energy Savings (PES) index, which represents the savings of a cogeneration unit with respect to the production of heat and electricity separately in a reference plant. As mentioned before, this index is also defined in the Spanish Royal Decree $616 / 2007$ of May $11^{\text {th }}, 2007$. It is evaluated once per year, and any cogeneration system with an installed capacity of more than $1 \mathrm{MWe}$ will be considered as highly efficient if the Primary Energy Savings are at least the $10 \%$ with respect to the separate generation.

$$
P E S=\left(1-\frac{1}{\frac{\mu Q_{C H P}}{\mu Q_{R e f}}+\frac{\mu E_{C H P}}{\mu E_{R E f}}}\right) \cdot 100
$$

Where:

- $\mu Q_{C H P}$ is the cogeneration heat efficiency, defined as the annual useful heat output $\left(Q_{C H P}\right)$ divided by the fuel input $\left(F_{C H P}\right)$, used to produce the sum of useful heat and electricity from cogeneration.

$$
\mu Q_{C H P}=\frac{Q_{C H P}}{F_{C H P}}
$$

- $\mu Q_{R e f}$ is the efficiency reference value for separate heat production. This value can be obtained from Regulation 2015/2402, and it depends on the cogeneration unit construction date and on the fuel type used. For the system considered in this work the value is 0.87 .

- $\mu E_{C H P}$ is the cogeneration electrical efficiency, defined as the annual electricity from cogeneration $\left(E_{C H P}\right)$ divided by the fuel input $\left(F_{C H P}\right)$, used to produce the sum of useful heat and electricity from cogeneration.

$$
\mu E_{C H P}=\frac{E_{C H P}}{F_{C H P}}
$$


- $\mu E_{R e f}$ is the efficiency reference value for separate electricity production. It can also be obtained from Regulation 2015/2402, and for the cogeneration plant considered has a value of 0.53 .

To obtain the PES index, Directive 2012/27/UE suggests using Decision 2008/952/EC, which establishes detailed guidelines for the implementation and application of Annex II. In the Directive considered, heat and electricity can be generated in CHP or non-CHP mode. According to Decision 2008/952/EC, NonCHP electricity means the electrical energy generated by a cogeneration unit in a reporting period when no related heat is produced by the cogeneration process, or part of the heat produced cannot be considered as useful heat.

On the other hand, a cogeneration unit is said to be operating in full cogeneration mode when it is operating with the maximum heat recovery technically possible from the cogeneration unit itself. The heat must be produced at the pressure and temperature levels required for the specific useful heat demand or market. In such case, all the heat and electricity would be generated in cogeneration mode (QCHP and ECHP). In cases where the plant does not operate in full cogeneration mode it is necessary to identify the electricity and heat not produced in cogeneration mode (QNon-CHP and ENon-CHP), to separate it from the generated in CHP mode (see Figure 4-5).

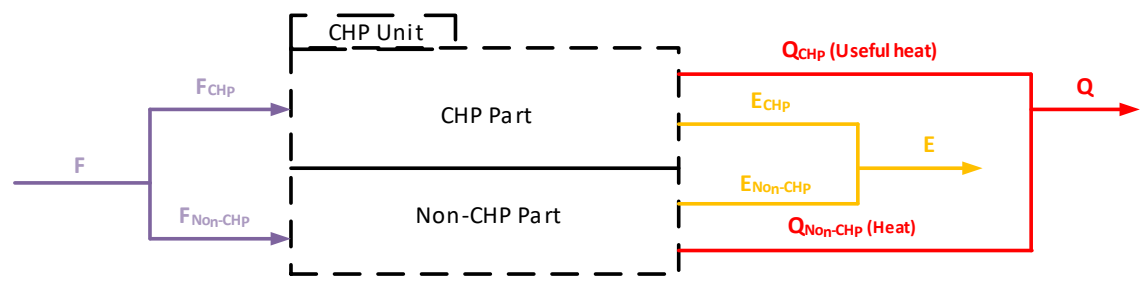

Figure 4-5. Parts of a cogeneration unit.

To obtain the electricity from cogeneration mode the first step is to calculate the overall efficiency $\left(\mu_{G}\right)$ of the cogeneration unit, which is defined as the CHP energy output (total electricity $(\mathrm{E})$ and useful heat $\left(Q_{C H P}\right)$ ) divided by the fuel input to the cogeneration unit. Such index is evaluated in a yearly basis.

$$
\mu_{G}=\frac{E+Q_{C H P}}{F}
$$

Considering the case study features, if the global efficiency of the plant is equal or greater than $75 \%$, then it will be considered that all the electrical power 
is generated in the CHP part. However, if the efficiency goes below 75\%, then the cogeneration unit will be split into two virtual parts (CHP and non-CHP part).

$$
\begin{array}{r}
E_{C H P}=\left\{\begin{array}{rr}
E-E_{\text {non-CHP }}, & \mu_{G}<75 \% \\
E, & \mu_{G} \geq 75 \%
\end{array}\right. \\
F_{C H P}=\left\{\begin{array}{rr}
F-F_{\text {non-CHP }}, & \mu_{G}<75 \% \\
F, & \mu_{G} \geq 75 \%
\end{array}\right.
\end{array}
$$

When the overall efficiency goes below $75 \%$, plant operators must check whether the plant works in full cogeneration mode during certain periods of time evaluating the system efficiency for such periods. Thus, they must measure the useful heat and electrical energy during such periods to obtain the actual "power-to-heat ratio" ( $\left.C_{\text {actual }}\right)$.

$$
C_{\text {Actual }}=\frac{E}{Q_{C H P}}
$$

With this value, it is possible to compute which part of the electricity generated during the reporting period is recognized as CHP electricity. If the actual 'power-to-heat ratio' cannot be calculated for some reason, the design 'power-to-heat ratio' given by Directive 2012/27/UE can be used instead. For the cogeneration plant considered the value is 0.45 .

$$
E_{C H P}=C_{\text {Actual }} \cdot Q_{C H P}
$$

With the CHP electricity computed, the non-CHP electricity can be obtained directly and, with this value and the "plant specific efficiency value for electricity production" $\left(\mu_{E}\right)$, the non-CHP fuel consumption can be computed, which is necessary to obtain the Primary Energy Savings.

$$
F_{\text {Non-CHP }}=\frac{E_{\text {Non-CHP }}}{\mu_{E}}
$$

Where:

$$
E_{\text {Non }-\mathrm{CHP}}=E-E_{C H P} \quad \mu_{E}=\frac{E}{F}
$$




\subsection{Optimization remarks}

The overall system description presented in the first part of this chapter, reveals the strong coupling existing between the proposed sugar extraction process and its cogeneration unit. Thus, in this case, an independent sequential study is not advisable. Furthermore, the slow process inertia when changes in the operational conditions are performed suggests that dynamic features must be considered in order to optimize the whole system operation. Therefore, this case study can be considered as a perfect candidate to test the methodology proposed in Chapter 3.

Having reviewed the Spanish electricity market and the legislation regarding the efficient use of cogeneration systems, we have done some assumptions to implement such information in the optimization problem. Regarding the electricity market:

- Given that accessing directly to the market requires an expert in the staff, which is something very difficult to find in a medium-size company not dedicated to trade electricity, we have assumed that the company can operate in the market only through a retailer. Thus, one optimization objective is the computation of the electricity commitment for a certain day that will be provided to the retailer.

- We have an accurate forecast of the market electricity price for the next day to the one considered.

- Given that we have a cogeneration unit, we can send bids for buying and selling electricity in the market for each hour. However, we cannot sell and buy electricity at the same time.

- In the buying price, the taxes term will be neglected in comparison to the energy term.

With respect to the legislation, due to its complexity, and to make the work comparable to other studies in the field, we have ignored the subsidies that would correspond to the considered plant, considering only the electricity market price. However, given that being a high-efficiency cogeneration plant gives priority in the dispatch and helps to improve the energy efficiency of the whole system, we have included the Primary Energy Savings index in the optimization problem as a measurement of the plant efficiency. 
Furthermore, following the methodology proposed in Chapter 3, after having studied the case study framework, we are ready to define the optimization aim, decision variables and operational constraints (please, see Figure 4-2 to facilitate the follow-up of the discussion).

Regarding the optimization aim, as it was mentioned before, the sugar extraction process is a seasonal business in Spain. A common way of working is fixing the sugar production objective before starting the campaign, and consequently, the beet needed, considering an average Brix and purity values during the whole campaign. If profit is defined as the difference between the incomes from sugar sales and the costs expended to produce it, having fixed the total amount of sugar that will be produced; the only way to increase profit is by reducing costs, which can be divided into operating and fixed costs. If we focus on minimizing the operating costs, such strategy may lead to a sharp beet processing rate reduction, and therefore, to an extension of the campaign duration and an increment of the fixed costs. Thus, to compensate such behavior, we propose the minimization of the specific operating cost over an accumulated time $T$ instead, which can be defined as the cost of the energy needed to process a certain amount of beet during that time. From a mathematical point of view, the dynamic optimization problem will be defined for a prediction horizon $T$, and therefore, the minimization of the integral of such function will be considered.

$$
\min _{u} \text { Specific Cost }:=\int_{0}^{T} \frac{\text { Operating } \operatorname{Costs}(t)}{\text { Beet processing rate }(t)} d t
$$

To minimize the proposed objective function, one variable that we can clearly modify is the beet processing rate $\left(W_{B S t o u t}\right)$, which indirectly refers to the sugar production rate. Furthermore, as we saw in Section 4.1, we can manipulate the power generated in the turbines $\left(E_{T u}\right)$, therefore, the difference between such power and the beet-sugar process electricity demand $\left(E_{P}\right)$, can be traded on the electricity market. This is the definition of the electricity committed $\left(E_{C}\right)$, and it has been proposed as another decision variable. Furthermore, manipulating the thermodynamic conditions of the steam generated in the cogeneration unit can considerably modify the energy efficiency of the system, and for that purpose, the overheated steam temperature 
obtained in boilers, and the pressure of the saturated steam sent to the evaporation stage have been included as decision variables too $\left(T_{S B o}, P_{S B o}\right)$.

With respect to the operating constraints, according to the description made in Section 4.1, the time beet spends stocked before being treated is a key variable to the system productivity $\left(\tau_{S T}\right)$, and therefore, it must be included in the optimization problem. Besides, as it was also mentioned in such section, the steam generated in the evaporation stage is used to feed many other parts of the sugar extraction process, being the crystallization section the most consuming stage. In our particular case, that section uses the steam provided by the fourth evaporation stage, and hence, maintaining its operating pressure above a desired bound is key to the correct functioning of the whole system $\left(P_{I V}\right)$. Finally, the legislation compliance will be added ensuring that the Primary Energy Savings index is always above the lower established bound (PES). 



\section{Chapter 5}

\section{Integral System Model of a Beet- Sugar Factory}

In this chapter, we apply the methodology discussed in Chapter 3 to obtain a model that is suitable for dynamic optimization using the simultaneous approach. To do so, we will take advantage of a much highly detailed simulator obtained previously, referenced as simulation module in Section 3.4, which will be used as "real plant" to obtain all the necessary data to build the optimization model and to test its performance. Details of the simulation module can be found in Appendix B.

\subsection{Inputs and outputs selection}

Following the discussion set in Section 3.2, the first thing to do to obtain the model, is to select its inputs and outputs. In the last section of the previous chapter, we set the optimization decision variables which can now be defined as the model inputs:

1. Beet processing rate $\left(W_{\text {BStOut }}\right)[370-430 \mathrm{~T} / \mathrm{h}]$.

2. Electricity power generation in turbines $\left(E_{T u}\right)[5-11 \mathrm{MW}]$.

3. Evaporation working pressure $\left(P_{\text {SSaOut }}\right)[2.2-3.0$ barA $]$. 
4. Superheated steam temperature obtained in boilers $\left(T_{S B o}\right)[360-420$ $\left.{ }^{\circ} \mathrm{C}\right]$.

Note the reader that we have chosen the electricity generated in turbines as input variable instead of the electricity commitment. Both variables are tightly related, and we have made this change to improve the clarity of the discussion.

Now, to select the model outputs, we need to have previously defined the optimization aim and the operational constraints. The optimization aim was defined as the minimization of the process specific energy cost for a period $T$, which mathematically can be described as follows:

$$
\text { Specific Cost }=\int_{0}^{T}\left(\frac{\frac{W_{G}(t)}{d_{G}} \cdot H H V_{G} \cdot P r_{G}-\left(\frac{E_{C}(t)}{3600} \cdot P r_{e}(t)\right)}{W_{B S t o u t}(t)}\right) d t
$$

In (5.1), the numerator represents the difference between the natural gas cost used in the boilers and the incomes obtained by selling the electricity surplus to the external grid. In the first term, since the natural gas cost must be expressed in [ $€ / \mathrm{kWh}$, the natural gas flow $\left(W_{G}\right)$ is divided by its density $\left(d_{G}\right)$ and multiplied by its Higher Heating Value $\left(H H V_{G}\right)$ and the natural gas Price $\left(\operatorname{Pr}_{G}\right)$.

In the second term, the electricity committed with the market is represented $\left(E_{c}\right)$, which will be the difference between the electricity generated in turbines $\left(E_{T u}\right)$ and the electricity consumed by the main process $\left(E_{p}\right)$. If electricity is sold to the market, this term will be positive, and since it means a cost reduction, the whole term will represent a negative contribution to the cost function. Otherwise, if the electricity is bought, the cost will increase. To express everything normalized with the same time scale $[€ / s]$, the electricity commitment term is divided by 3600 .

In the denominator, the beet processing rate is found $\left(W_{B S t O u t}\right)$. Finally, the integral of this quantity is computed along the whole prediction horizon T. A full description of the model variables and parameters can be found in Appendix $A$. Therefore, from this expression we can conclude that we need to obtain the value of the natural gas mass flow $\left(W_{G}\right)$, and the electricity consumed in the factory $\left(E_{P}\right)$.

With respect to the operational constraints, we must: 
- Meet the legislation related to the efficient operation of cogeneration plants. To do so, we need to compute the Primary Energy Savings (PES).

- Ensure a stock of beet between limits. This can be measured in two different ways: One is measuring the remaining beet in the storage zone $\left(m_{S t}\right)$; and the other is computing the average residence time the beet spends inside the storage zone $\left(\tau_{s t}\right)$. The last is defined as the accumulated beet over the beet processing rate, and if it is too high, the beet can rot. On the other hand, it shows how long the process can be operated at a specified production rate without running out of beet, if the beet input drops to zero. Since the residence time gives more information about the beet storage, and it is easier to constrain, it will be used in the optimization problem and, therefore, it will be an output of the model.

- Maintain the pressure of the $4^{\text {th }}$ evaporation effect $\left(P_{I V}\right)$ above the desired limit to ensure that enough steam is available for other parts of the process.

Finally, since one of the objectives of any CHP system is to generate the amount of heat energy demanded by the main process, the heat energy consumed by the sugar factory $\left(Q_{P}\right)$ is also considered as an output of the model.

To sum up, a list of the desired output variables is shown below:

1. Electricity consumption of the sugar factory $\left(E_{P}\right)$.

2. Natural gas mass flow rate needed to operate the whole process $\left(W_{G}\right)$.

3. Primary Energy Savings index (PES).

4. Average time beet spends in the storage zone $\left(\tau_{S t}\right)$.

5. Steam pressure inside the fourth effect of the evaporation $\left(P_{I V}\right)$.

6. Heat energy consumption of the sugar factory $\left(Q_{P}\right)$.

In the previous chapter we reviewed the framework of the case study presented in this thesis. Now, in this chapter, we will look for a model tractable from a computational point of view that represents the closed-loop behavior of the integrated main process-CHP system. To do so, we will apply step by step the methodology depicted in Section 3.2, obtaining as a result a DAE model that combines first-principles equations with empirical relationships. Once validated, this model will be used in the following chapter, where the implementation of 
the dynamic optimization problem will be detailed. The contributions showed in this chapter were published in (Pablos et al., 2019).

\subsection{First-principles equations}

Once the model inputs and outputs have been specified, equations that relates them are sought. As direct relations are sometimes very difficult or even impossible to find, the whole system is divided into different control volumes in order to make the modeling process easier. The selection of such control volumes depends on the equations available, the knowledge of the relationships between variables, and the availability of process measurements. The division taken is shown in Figure 5-1, where control volumes are emphasized with black lines. However, this is not the only possibility, and other control volumes could have been chosen.

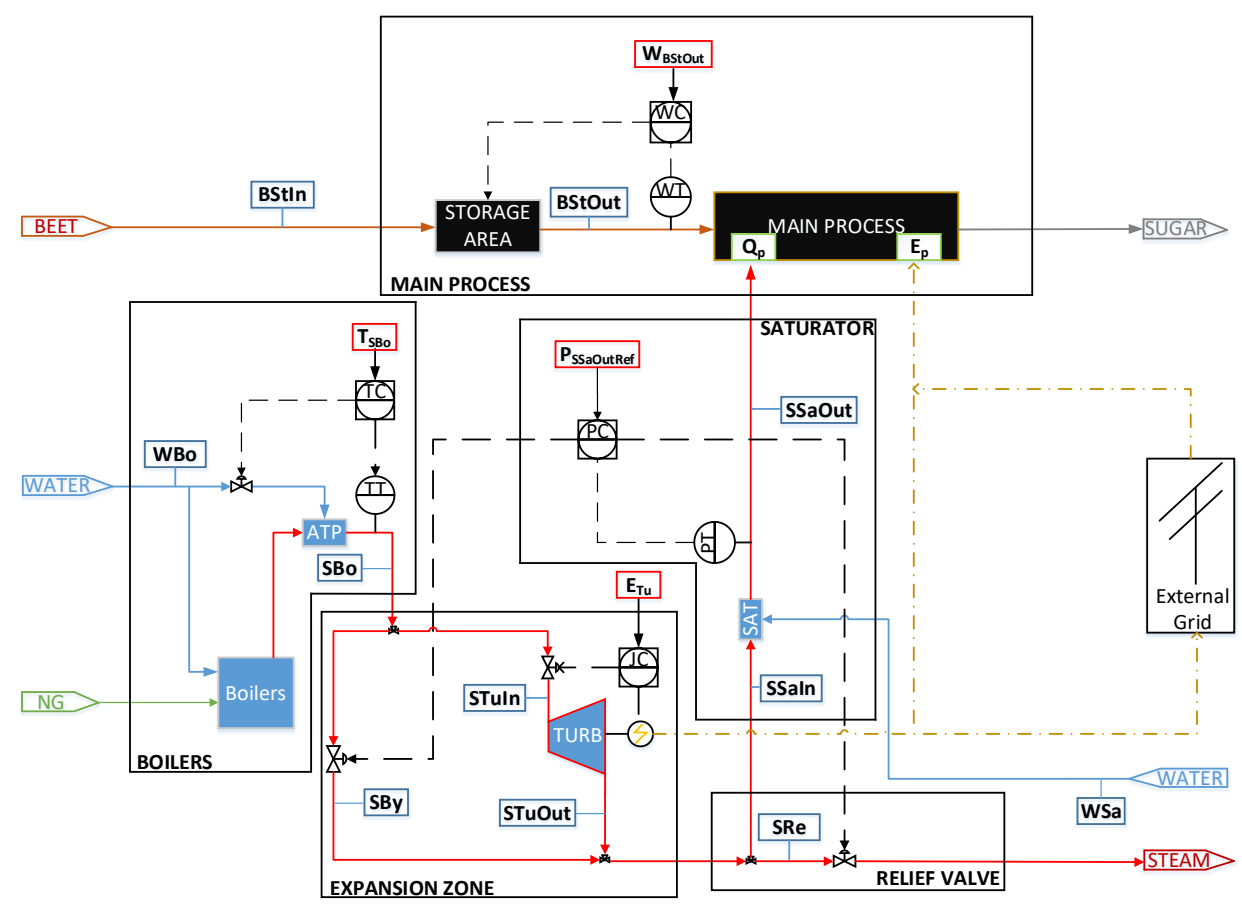

Figure 5-1. Control volumes selected for the purposed case study. 
Now, we need to analyze each control volume as a separated model with its own inputs and outputs. In this phase, the empirical equations are remarked but not yet defined.

\section{Main Process}

In this control volume, we include the storage area, where beet is piled up until is processed, and the beet-sugar industrial process. The aim is to obtain the heat and power energy consumption, the pressure inside the fourth evaporation effect, and the beet available in the storage zone.

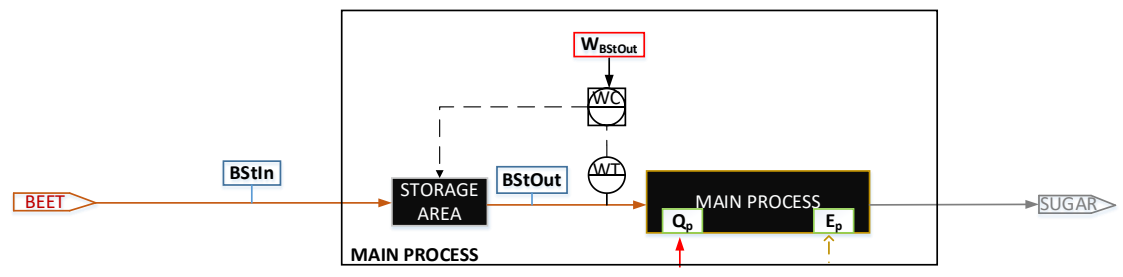

Figure 5-2. Main process control volume scheme.

Regarding the main process heat consumption $\left(Q_{p}\right)$, although it depends on many variables, it has been assumed that the most influential ones are the evaporation working pressure $\left(P_{\text {SSaOut }}\right)$, because of its implication in the performance of the evaporators (Urbaniec, 1989), and the production rate $\left(W_{\text {BStout }}\right)$. To avoid modeling the whole process, an empirical relationship between these variables will be searched. Given that, a priori, we ignore the nature of such relationship, but we know that its dynamics must be considered, we pose a general dynamic implicit equation to describe its behavior:

$$
f\left(\frac{d Q_{p}^{n}(t)}{d t^{n}}, \frac{d Q_{p}^{n-1}(t)}{d t^{n-1}}, \ldots, \frac{d Q_{p}(t)}{d t}, Q_{p}(t), W_{\text {BStout }}(t), P_{\text {SSaOut }}(t)\right)=0
$$

On the other hand, according to (Frankenfeld and Voss, 2004), it can be assumed that for the operational range considered, among all the process variables, electricity power consumption $\left(E_{p}\right)$ depends mainly on the beet processing rate: 


$$
f\left(\frac{d E_{p}^{n}(t)}{d t^{n}}, \frac{d E_{p}^{n-1}(t)}{d t^{n-1}}, \ldots, \frac{d E_{p}(t)}{d t}, E_{p}(t), W_{\text {BStout }}(t)\right)=0
$$

In the same fashion, a simple expression is sought based on experimental data that represents how pressure inside the fourth evaporation effect varies during the process operation. In the factory studied, the beet processing rate and the evaporation working pressure are the variables that most affect this pressure. If production is increased and the evaporation working pressure is maintained constant, the water removed from the juice will not be enough to cope with the process steam demand, and eventually the pressure of every evaporation effect will drop. Instead, if the working pressure is increased, but the evaporation input juice flow remains constant, more water than necessary will be removed, and the pressure of the evaporators will increase. Other variables may affect pressure inside the evaporators, but they are rarely changed during normal process operation and their effect is not that significant, so they have not been considered:

$$
f\left(\frac{d P_{I V}^{n}(t)}{d t^{n}}, \frac{d P_{I V}^{n-1}(t)}{d t^{n-1}}, \ldots, \frac{d P_{I V}(t)}{d t}, P_{I V}(t), W_{B S t o u t}(t), P_{\text {SSaOut }}(t)\right)=0
$$

With respect to the beet available in the storage zone, as it was mentioned before, instead of the accumulated beet, the average residence time beet spends there is the desired output $\left(\tau_{S t}\right)$, which has been defined as follows:

$$
\tau_{S t}(t)=\frac{m_{S t}(t)}{W_{\text {BStout }}(t)}
$$

where the accumulated beet $\left(m_{S t}\right)$ can be obtained with a mass balance between the storage area input and output. Since the beet input and output flow are measured in $[T / h]$ and the rest of the model variables are in seconds, a change of units must be done dividing the difference by 3600 :

$$
\frac{d m_{S t}(t)}{d t}=\frac{W_{B S t I n}(t)-W_{B S t O u t}(t)}{3600}
$$


Looking at equations (5.2) - (5.6), performing a degree of freedom analysis, and considering that the beet arrival $\left(W_{B S t I n}\right)$ is a known parameter, there are two degrees of freedom. Beet processing rate is a known input that can be fixed as an input, and the other one can be the evaporation working pressure, which must be computed using other control volumes.

\section{Saturator}

The saturator control volume (see Figure 5-3), includes the saturator itself and the split-range pressure control system used to control the evaporation working pressure.

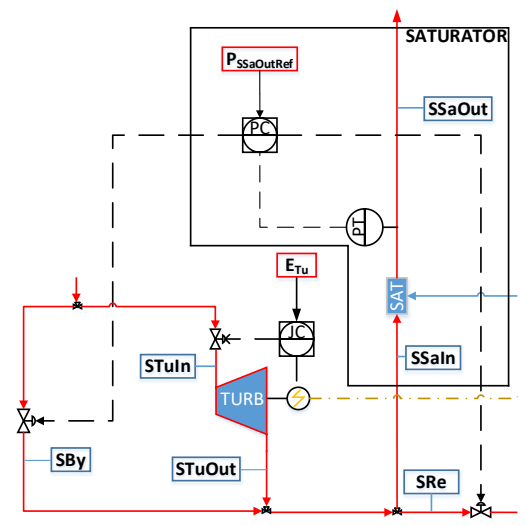

Figure 5-3. Saturator control volume scheme.

Here are listed the equations that describe its functioning:

$$
\begin{gathered}
e(t)=P_{\text {SSaOutRef }}(t)-P_{\text {SSaOut }}(t) \\
v(t)=k p \cdot e(t)+v_{i}(t) \\
\frac{d v_{i}(t)}{d t}=\frac{k p}{T i} \cdot e(t)
\end{gathered}
$$




$$
\begin{gathered}
O p_{B y}(t)=\max \left(0, \min \left(100, \frac{v_{\max }}{v_{\max }-45} \cdot(v(t)-45)\right)\right) \\
O p_{R e}(t)=\max \left(0, \min \left(100, \frac{-v_{\max }}{45} \cdot(v(t)-45)\right)\right)
\end{gathered}
$$

where:

$$
0 \leq v(t) \leq 100
$$

The split-range controller must decide the opening of the bypass and relief valves, considering that only one of them can be opened at the same time, or both must be closed. Equations (5.10) and (5.11) are used to find the opening of such valves as a function of $v(t)$, which is obtained as the output of the PI controller equation (5.8). Therefore, if the value of $v(t)$ is greater than 45 the bypass will be opened, and the relief valve will be closed. If the value of $v(t)$ is 45 , both will be closed, and if it is less than 45 the relief valve will be the only valve opened.

Regarding the saturator equipment, there, the expanded steam obtained from the turbines is saturated with a water flow before it is used in the evaporation section. This unit can be easily modeled using first-principles equations:

$$
\begin{gathered}
W_{\text {SSaOut }}(t)=W_{\text {SSaIn }}(t)+W_{\text {WSa }}(t) \\
W_{\text {SSaOut }}(t) \cdot H_{\text {SSaOut }}(t)=W_{\text {SSaIn }}(t) \cdot H_{\text {SSaIn }}(t)+W_{\text {WSa }}(t) \cdot H_{W S a} \\
W_{\text {SSaOut }}(t)=\frac{Q_{p}(t)}{H_{\text {SSaOut }}(t)}
\end{gathered}
$$

Finally, in this control volume, we can also consider the relationship between the steam pressure and specific enthalpy at the saturator output, which can be interpolated using data extracted from thermodynamic tables (Perry et al., 2015), and it is valid for a pressure interval from 1 to 3 bar. 


$$
H_{\text {SSaOut }}(t)=24.350 \cdot P_{\text {SSaOut }}(t)+2656
$$

In this control volume we have thirteen variables and nine equations, which gives us 4 degrees of freedom. Naturally, the reference of the split-range controller can be fixed as a known input $\left(P_{S S a O u t R e f}\right)$. Furthermore, the heat consumed by the process $\left(Q_{p}\right)$ can be computed using the equations listed for the main process. For the other two degrees of freedom, we have selected as inputs the mass flow and the specific enthalpy of the steam that enters in the saturator $\left(W_{\text {SSaIn }}, H_{\text {SSaIn }}\right)$. These variables must be computed using the relief valve control volume.

\section{Relief valve}

This control volume includes the relief valve itself, which is used for reducing an excess of pressure in the system, and the bifurcation that leads the expanded steam to the saturator or to the relief valve.

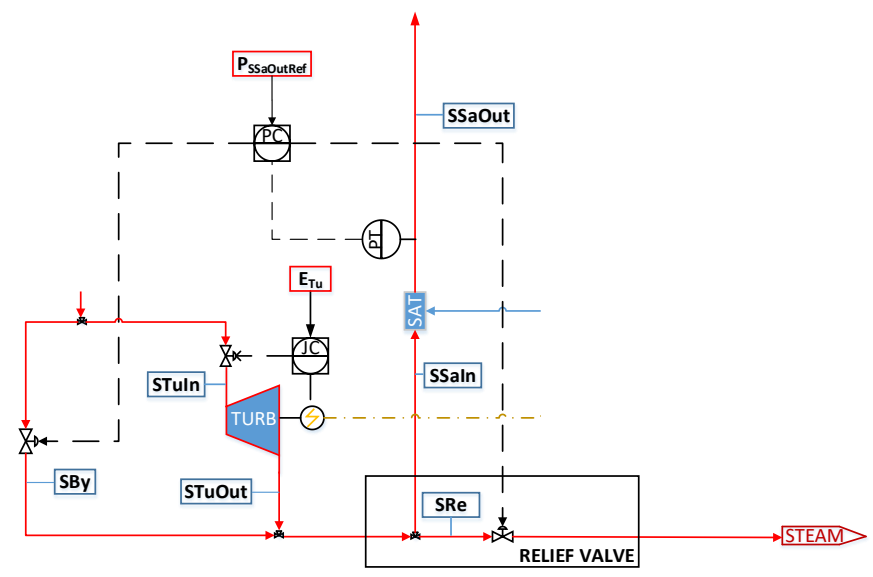

Figure 5-4. Relief valve control volume scheme.

It has been modeled as shown below:

$$
W_{S R e}(t)=K_{V_{R e}} \cdot \frac{O p_{R e}(t)}{100} \cdot \sqrt{P_{\text {SSaOut }}(t)^{2}-P_{\text {atm }}(t)^{2}}
$$




$$
\begin{gathered}
W_{\text {SBy }}(t)+W_{\text {STuIn }}(t)=W_{\text {SRe }}(t)+W_{\text {SSaIn }}(t) \\
\begin{array}{c}
W_{\text {SBy }}(t) \cdot H_{\text {SBy }}(t)+W_{\text {STuIn }}(t) \cdot H_{\text {STuOut }}(t) \\
=
\end{array} W_{\text {SRe }}(t) \cdot H_{\text {SRe }}(t)+W_{\text {SSaIn }}(t) \cdot H_{\text {SSaIn }}(t) \\
H_{\text {SRe }}(t)=H_{\text {SSaIn }}(t)
\end{gathered}
$$

It has been assumed that the pressure drop due to the pipes and the saturator is negligible, so the steam pressure at the output of the turbines is assumed to be equal to the evaporation working pressure:

$$
P_{\text {STuOut }}(t)=P_{\text {SSaOut }}(t)
$$

On the other hand, in equation (5.17) the steam mass flow at the turbines input $\left(W_{S T U I n}\right)$ is used instead of the steam mass flow at the turbines output $\left(W_{S T \text { U }}\right)$ because it has been assumed to be equal through the turbine.

$$
W_{\text {STuIn }}(t)=W_{\text {STuOut }}(t)
$$

Here, we have four equations for nine variables, therefore, we need to fix the value of five of them. The opening of the relief valve can be computed using Equation (5.11). The other four are the mass flow and specific enthalpy of the steam that leaves the boilers and the bypass valve in the expansion zone.

\section{Expansion zone}

It contains three identical turbines working in parallel and a bypass recirculation for steam (see Figure 5-5). To simplify the problem, only one large steam turbine has been modeled, in such a way that it will consume the same amount of steam to generate the same amount of power as the sum of the other three. 


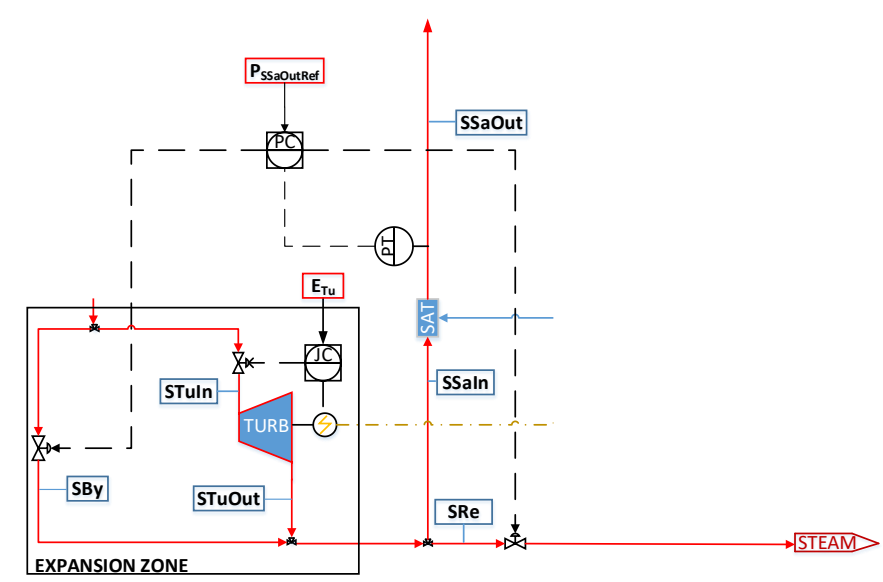

Figure 5-5. Expansion zone control volume scheme.

The first equation considered in this control volume is a mass balance, where the steam mass flow generated in the boilers must be equal to the steam mass flow that goes through the turbine and the bypass valve:

$$
W_{S B o}(t)=W_{S B y}(t)+W_{S T u I n}(t)
$$

The bypass valve can be modeled using a similar expression as in (5.16), where the boilers steam pressure $\left(P_{S B O}\right)$ is assumed to be constant during normal process operation and, given that it can be controlled using a $\mathrm{PI}$ controller, known:

$$
W_{S B y}(t)=K v_{B y} \cdot \frac{O p_{B y}(t)}{100} \cdot \sqrt{P_{S B o}^{2}-P_{S S a O u t}(t)^{2}}
$$

With respect to the turbines, the steam mass flow passing through $\left(W_{S T u I n}\right)$ can be calculated using the expression obtained from (Chaibakhsh and Ghaffari, 2008), where $K_{T u}$ is a parameter that must be adjusted from experimental data.

$$
W_{\text {STuIn }}(t)=\frac{K_{\text {Tu }}}{\sqrt{T_{\text {STuIn }}(t)}} \sqrt{P_{\text {STuIn }}(t)^{2}-P_{\text {STuOut }}(t)^{2}}
$$

In the same fashion as in (5.15), the specific enthalpy of the turbine input and output steam can be obtained interpolating the thermodynamic tables 
found in the literature. The obtained function is valid for every pressure when the temperature is higher than $283^{\circ} \mathrm{C}$.

$$
\begin{gathered}
H_{\text {STuIn }}(t)=2355-1.490 \cdot P_{\text {STuIn }}(t)+2.291 \cdot T_{\text {STuIn }}(t) \\
H_{\text {STuout }}(t)=2355-1.490 \cdot P_{\text {STuout }}(t)+2.291 T_{\text {STuout }}(t)
\end{gathered}
$$

Let us now assume that the steam expansion through the turbines is isentropic and adiabatic, the following expression can be used therefore to model the turbine thermodynamics, where $\mathrm{k}$ is the polytrophic expansion factor for steam:

$$
\frac{T_{\text {STuout }}(t)}{T_{\text {STuIn }}(t)}=\left(\frac{P_{\text {STuout }}(t)}{P_{\text {STuIn }}(t)}\right)^{\frac{k-1}{k}}
$$

And the power generated can be obtained using the following equation:

$$
E_{T u}(t)=\mu_{T u} \cdot W_{\text {STuIn }}(t) \cdot\left(H_{\text {STuIn }}(t)-H_{\text {STuOut }}(t)\right)
$$

Finally, valves have been assumed to be completely insulated, so they can be considered as adiabatic. If so, the enthalpy before and after the bypass valve and the turbine control valve must remain constant, and both must be equal to the specific enthalpy of the steam produced in boilers.

$$
\begin{gathered}
H_{S T u I n}(t)=H_{S B o}(t) \\
H_{S B y}(t)=H_{S B o}(t)
\end{gathered}
$$

To sum up, in the expansion zone model, we have thirteen variables and nine equations which yields four degrees of freedom. The electricity generated in the turbines $\left(E_{T u}\right)$ can be clearly fixed as an input as it is a set-point. On the other hand, the opening of the bypass valve $\left(O p_{B y}\right)$, and the saturator outlet pressure $\left(P_{S S a O u t}\right)$, can be computed using the saturator control volume. Hence, only one more equation must be fixed, and we have selected the boilers steam specific enthalpy $\left(H_{S B O}\right)$, which can be obtained from the boilers control volume. 


\section{Boilers}

This control volume includes the preheating water system, the boilers themselves, and the overheating steam system, where a PI controls the overheated steam temperature providing a water flow into a heat exchanger called attemperator (ATP).

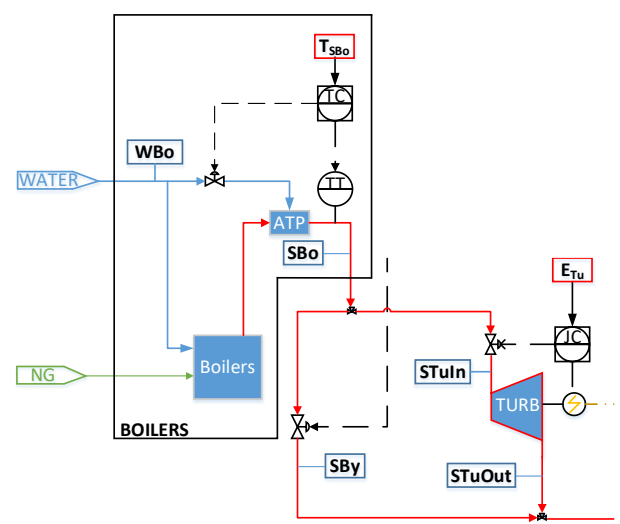

Figure 5-6. Boilers control volume scheme.

To obtain the specific enthalpy needed, we can use the same expression proposed in (5.25) and (5.26):

$$
H_{S B o}(t)=2355-1.490 \cdot P_{S B o}+2.291 \cdot T_{S B o}(t)
$$

Considering that the boilers steam temperature is a set-point, with this expression we can close the model and obtain the desired outputs mentioned for the main process control volume. Nevertheless, we still need to compute the natural gas needed to run the whole process $\left(W_{G}\right)$, and the Primary Energy Savings (PES).

The former can be obtained modeling the boilers, however, including a detailed model can be unsuitable for the optimization problem, so given that we can compute the steam demanded from the whole system and its thermodynamic properties, we can search for an empirical model that links the steam produced and the natural gas used in boilers. 


$$
f\left(\frac{d W_{G}^{n}(t)}{d t^{n}}, \frac{d W_{G}^{n-1}(t)}{d t^{n-1}}, \ldots, \frac{d W_{G}(t)}{d t}, W_{G}(t), W_{S B o}(t), H_{S B o}(t)\right)=0
$$

Directive 2012/27/UE

To compute the PES index, we have used Equation (4.1) assuming that the efficiency of our cogeneration plant is always above $75 \%$ (see Equation (4.4)). This can be easily forced in the optimization problem as a constraint. Therefore, we can consider that all the heat, electricity, and fuel is generated in " $\mathrm{CHP}$ mode". The reference values needed in (4.1) were obtained from Regulation 2015/2402. Following Decision 2008/952/EC, we have computed the heat obtained in the cogeneration system as the difference between the steam heat at the inlet of the saturator and the water heat used in the boilers, assuming that water losses are negligible.

$$
Q_{C H P}(t)=W_{S S a I n}(t) \cdot\left(H_{S S a I n}(t)-H_{W B o}\right)
$$

On the other hand, the fuel energy can be obtained using Equation (5.34), and the electricity generated in the plant can be considered to be the one fixed in the turbines:

$$
\begin{gathered}
F_{C H P}(t)=W_{G}(t) \cdot L H V_{G} \\
E_{C H P}(t)=E_{T u}(t)
\end{gathered}
$$

Note the reader that all the variables needed have been already computed in the previous sections.

\subsection{Black-box models identification}

Following the methodology described in Section 3.2, and considering that our plant is simulated, we can assume that all the process variables needed for identification are measured, trusty, and available. Therefore, after having dealt with the first-principles equations of the model, now, we must identify each of 
the black-box equations that were remarked in Section 5.2, specifically, equations (5.2-5.4 and 5.32). To do so, first, we must run some experiments to check the linearity of each relationship, analyze its dynamic response, and later, we need to select a structure for each equation and perform a regression analysis.

\section{Beet-sugar factory heat demand $\left(Q_{p}\right)$}

It was defined in equation (5.2) as a dynamic equation which depends on the beet processing rate and the evaporation working pressure. To check linearity, some experiments were carried out trying different values for both input variables throughout its working range and measuring the heat demand in the stationary state. The experiments were performed independently for each input variable, maintaining the other one constant. The results of such experiments are shown in Figure 5-7. As the reader can see, the relationship of pressure and production with heat consumption can be assumed to be linear in the steady state for the operational point considered. Based on that conclusion, we have assumed that this condition holds for other points, and it will be tested later in the validation step.
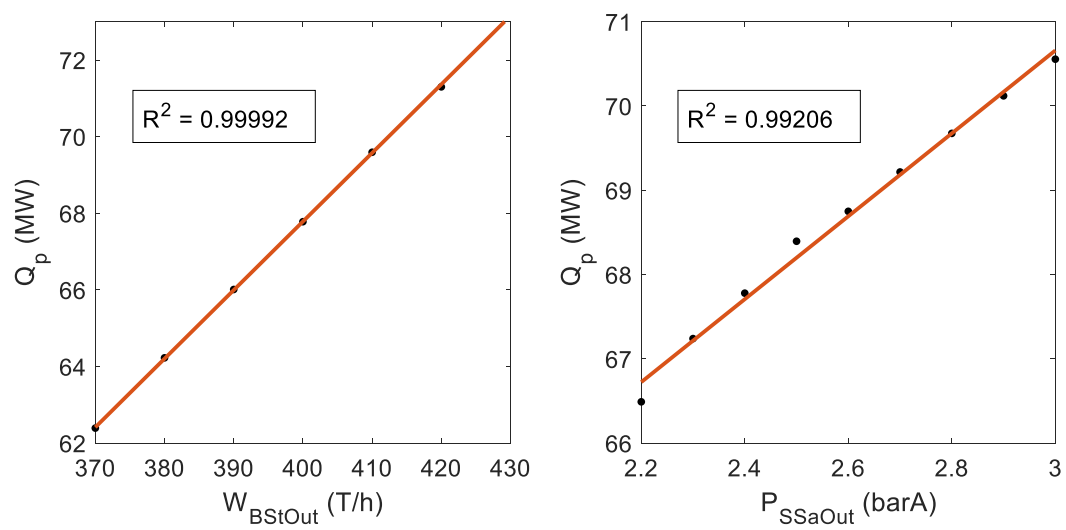

Figure 5-7. Experiments carried out to show linearity dependence between $Q_{p}$ and $W_{\text {BStOut }}$ (left) and $P_{\text {SSaOut }}$ (right).

Once linearity has been studied, the step response for both inputs is shown in Figure 5-8. A second order dynamic response with a delay of almost one hour and a settling time of three hours can be discerned for $W_{B S t O u t}$. Regarding $P_{\text {SSaOut }}$, things are more complicated to deduct considering the step response 
during the first instants of the transitory period. Such behavior is due to the aggressive tuning of the split-range pressure controller, which tries to take the pressure to the new set-point in the shortest possible time. This is done in order to send steam to the system as quickly as possible if required. A settling time of one hour can be observed, and there is no delay in the response. If the first part of the transient is ignored, the step response behavior is close to a typical linear first order system, with no delay and a settling time of one hour. For the optimization problem considered, capturing the steady state is essential, but transients are also important if they expand in time. In the case of the heat energy consumption with respect to the evaporation working pressure, the transient overshoot is damped relatively quickly, so the model proposed for its identification should not be focused on this short response.
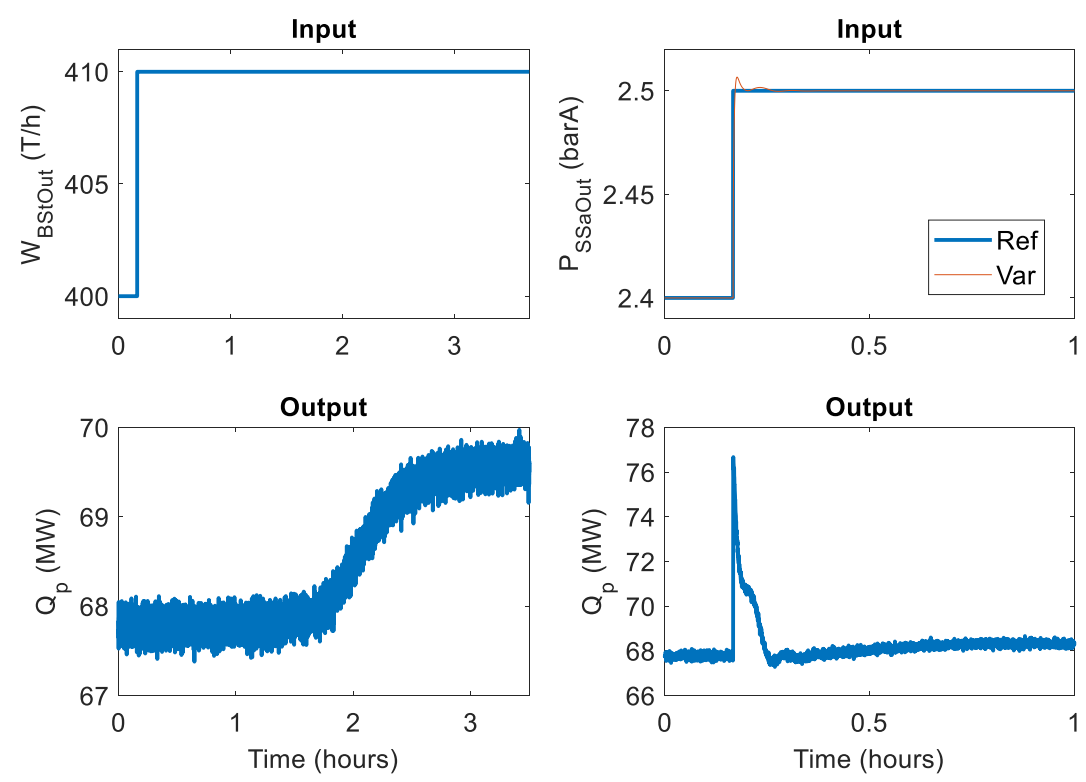

Figure 5-8. Dynamic response of $Q_{p}$ for a step in $W_{B S t O u t}$ (left); Dynamic response of $Q_{p}$ for a step in $P_{\text {SSaOut }}$ (right).

With all this information, a fourth order state-space model is proposed. For simplicity we have assumed the coefficients to be constant. This assumption will be validated during the validation step. To obtain the model, the MATLAB ${ }^{\circledR}$ system identification toolbox was used, and the resulting model is shown in equation (5.36). 


$$
\begin{gathered}
Q_{p}(t)=Q_{P_{e q}}+\Delta Q_{P}(t) \\
\dot{x}_{Q_{P}}(t)=\left[\begin{array}{cccc}
-1.077 & -8.815 \cdot 10^{-2} & 0.000 & 0.000 \\
6.250 \cdot 10^{-2} & 0.000 & 0.000 & 0.000 \\
0.000 & 0.000 & -5.521 \cdot 10^{-4} & -3.252 \cdot 10^{-4} \\
0.000 & 0.000 & 4.883 \cdot 10^{-4} & 0.000
\end{array}\right] x_{Q_{P}}(t) \\
+\left[\begin{array}{rr}
256.000 & 0.000 \\
0.000 & 0.000 \\
0.000 & 0.250 \\
0.000 & 0.000
\end{array}\right]\left[\begin{array}{cc}
\Delta W_{\text {BStout }}(t-3600) \\
\Delta P_{\text {SSaOut }}(t)
\end{array}\right] \\
\Delta Q_{P}(t)=\left[\begin{array}{llll}
172.400 & 1.744 & 5.629 \cdot 10^{-5} & 0.231
\end{array}\right] x_{Q_{P}}(t)
\end{gathered}
$$

\section{Beet-Sugar factory electricity power demand $\left(E_{p}\right)$}

In the previous section, the factory electricity power consumption was defined as a dynamic function of the beet processing rate (equation (5.3)). According to (Frankenfeld and Voss, 2004; Urbaniec, 1989), it has been assumed that for the operational range considered, its dependency can be considered as linear. Note that in the same fashion as the beet-sugar factory heat demand, this assumption will be tested in the validation section. The step response is shown in Figure 5-9.

From such Figure 5-9, a second order dynamic response can be inferred. Again, a delay of almost one hour appears between the input and the output, and a settling time of approximately three hours can be discerned. A state-space second order dynamic system is proposed, and the final model obtained is shown in Equation (5.37).

$$
\begin{gathered}
E_{p}(t)=E_{P_{e q}}+\Delta E_{P}(t) \\
\dot{x}_{E_{P}}(t)=\left[\begin{array}{cc}
-1.021 \cdot 10^{-3} & -8.359 \cdot 10^{-4} \\
4.883 \cdot 10^{-4} & 0
\end{array}\right] x_{E_{P}}(t) \\
+\left[\begin{array}{c}
0.125 \\
0
\end{array}\right]\left[\Delta W_{B S t o u t}(t-3600)\right] \\
\Delta E_{P}(t)=\left[\begin{array}{ll}
2.423 \cdot 10^{-2} & 0.127] x_{E_{P}}(t)
\end{array}\right.
\end{gathered}
$$



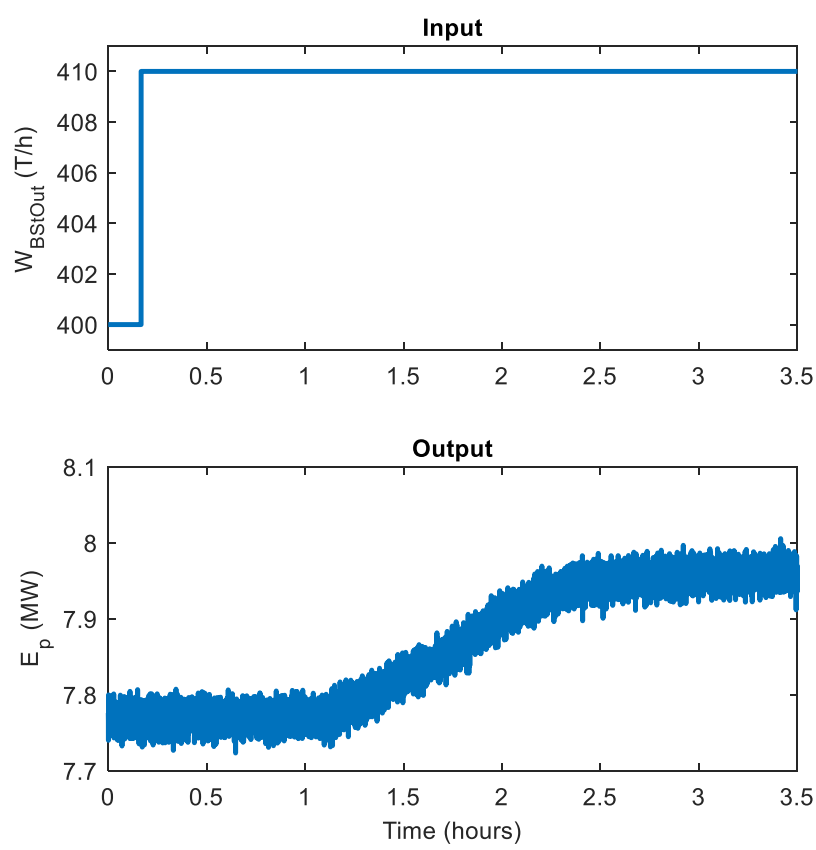

Figure 5-9. Dynamic response of $E_{p}$ for a step in $W_{B S t O u t}$.

Pressure inside the evaporation fourth effect $\left(P_{I V}\right)$

In equation (5.4), the pressure inside the evaporation fourth effect was defined as a dynamic function of the evaporation working pressure $\left(P_{S S a O u t}\right)$, and the beet processing rate $\left(W_{B S t O u t}\right)$. To study the linearity of $P_{I V}$ with respect to both inputs, different experiments were carried out changing the values of each input while the other remains constant and waiting until the stationary was reached. The results of these experiments are shown in Figure 5-10.

Again, considering the results shown in Figure 5-10 we have assumed that linearity holds for other points inside the operational range. In Figure 5-11, the dynamic response of $P_{I V}$ when step changes are introduced in $W_{B S t O u t}$ and $P_{\text {SSaOut }}$ is shown. As it can be observed, when the beet processing rate is increased, the pressure inside the fourth effect rises following a typical linear second order response. The delay and the settling time are the same as before with $Q_{p}$ and $E_{p}$. With respect to $P_{S S a O u t}$, a very similar response to the one seen for $Q_{p}$ is found. Again, if the first part of the transient state is ignored, the response of the system is close to a first order dynamic, there is no delay, and 
the settling time is one hour. Thus, with the same reasoning as the one used for the identification of $Q_{p}$, a MIMO fourth order state-space model is obtained using identification techniques:
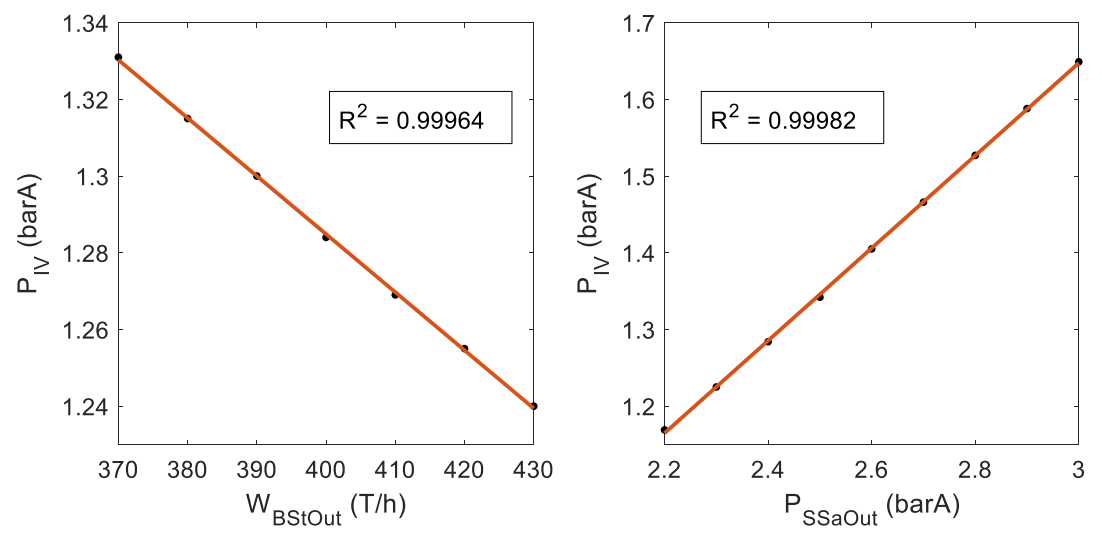

Figure 5-10. Results of the experiments carried out to show linearity dependence between $P_{I V}$ and $W_{B S t O u t}$ (left) and $P_{\text {SSaOut }}$ (right).

$$
\begin{aligned}
& P_{I V}(t)=P_{I V_{e q}}+\Delta P_{I V}(t) \\
& \dot{x}_{P_{I V}}(t)=\left[\begin{array}{cccc}
-0.309 & -5.434 \cdot 10^{-2} & 0.000 & 0.000 \\
6.250 \cdot 10^{-2} & 0.000 & 0.000 & 0.000 \\
-7.163 \cdot 10^{-4} & -3.915 \cdot 10^{-4} & 0.000 & 0.000 \\
4.883 \cdot 10^{-4} & 0.000 & 0.000 & 0.000
\end{array}\right] x_{P_{I V}}(t) \\
& +\left[\begin{array}{rr}
0.250 & 0.000 \\
0.000 & 0.000 \\
0.000 & 9.766 \cdot 10^{-4} \\
0.000 & 0.000
\end{array}\right]\left[\begin{array}{c}
\Delta W_{\text {BStout }}(t-3600) \\
\Delta P_{\text {SSaOut }}(t)
\end{array}\right] \\
& \Delta P_{I V}(t)=\left[\begin{array}{llll}
9.397 \cdot 10^{-2} & 0.122 & -1.449 \cdot 10^{-7} & -5.935 \cdot 10^{-4}
\end{array}\right] x_{P_{I V}}(t)
\end{aligned}
$$



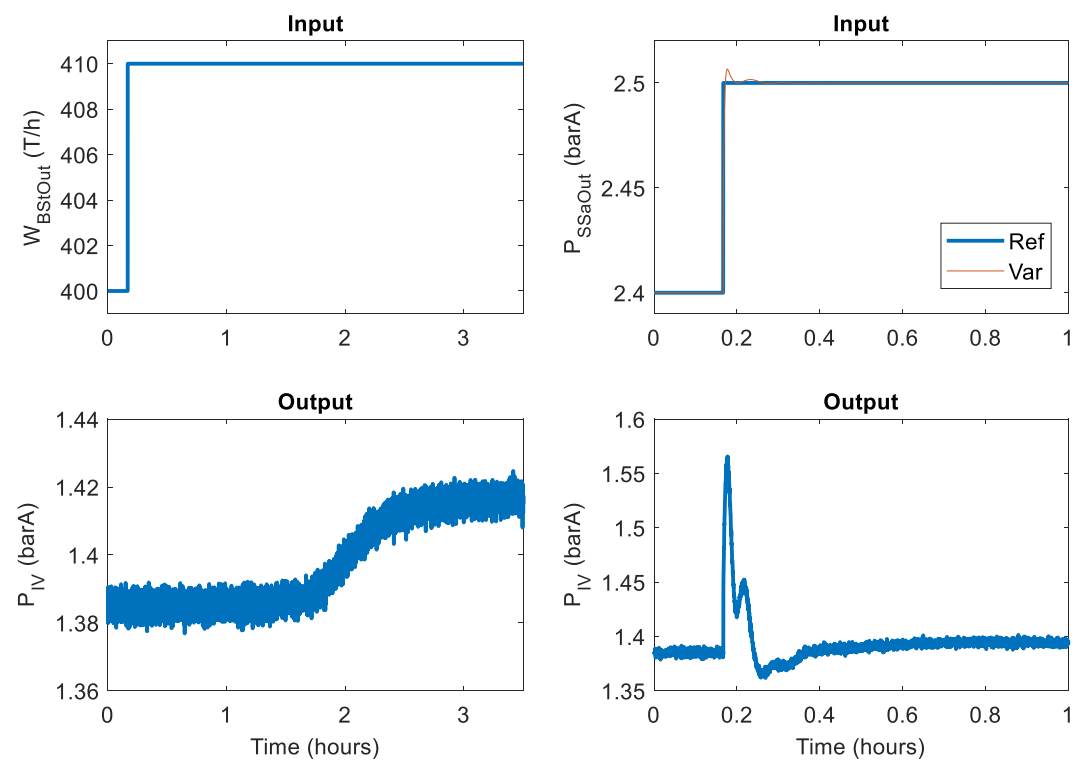

Figure 5-11. Dynamic response of $\mathrm{P}_{\mathrm{IV}}$ for step changes. $\mathrm{W}_{\mathrm{BStOut}}$ (Left); $\mathrm{P}_{\mathrm{SSaOut}}$ (Right).

Natural gas consumption $\left(W_{G}\right)$

In Equation (5.32) we defined the boilers natural gas consumption as a function of the mass flow and the specific enthalpy of the overheated steam produced. However, doing some experiments, we reached the conclusion that considering both inputs separately they were not linearly related to $W_{G}$. However, things changed when we used the heat $\left(Q_{S B o}\right)$ instead. The problem was that we could not measure the heat of such stream, therefore, we used the mass flow, temperature and pressure measures to compute it using equation (5.39).

$$
Q_{S B o}=W_{S B o} \cdot H_{S B o}
$$

In this case, to test linearity, since the heat of a current cannot be directly manipulated, we made some experiments in the stationary, changing the beet processing rate $\left(W_{B S t O u t}\right)$ and the power generated in the turbines $\left(E_{T u}\right)$, which are the most influential variables. From the results shown in Figure 5-12, it can be inferred that the relation between the superheated steam heat and the natural gas flow is almost linear in the stationary state. Note the reader that we are using a simulation, such a good regression would not be expected in real systems. 


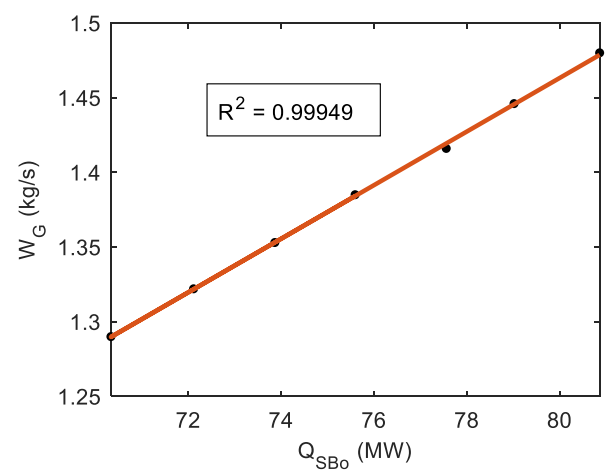

Figure 5-12. Linearity between $\mathrm{Q}_{\mathrm{SBo}}$ and $\mathrm{W}_{\mathrm{G}}$.

Regarding the dynamic relation between $Q_{S B o}$ and $W_{G}$, since a proper step cannot be carried out, apart from the lack of delay between them, little more information could be gathered from the results shown in Figure 5-13, so we proposed a second order state-space which will be evaluated in validation, and we identified the following model:

$$
\begin{gathered}
W_{G}(t)=W_{G_{e q}}+\Delta W_{G}(t) \\
\dot{x}_{W_{G}}(t)=\left[\begin{array}{cc}
-7.528 \cdot 10^{-2} & -2.828 \cdot 10^{-2} \\
-1.563 \cdot 10^{-2} & 0
\end{array}\right] x_{W_{G}}(t) \\
+\left[\begin{array}{c}
1.953 \cdot 10^{-3} \\
0
\end{array}\right]\left[\Delta Q_{S B o}(t)\right] \\
\Delta W_{G}(t)=\left[1.038 \cdot 10^{-3} \quad 2.308 \cdot 10^{-4}\right] x_{W_{G}}(t)+\left[2.101 \cdot 10^{-6}\right] u_{W_{G}}(t)
\end{gathered}
$$



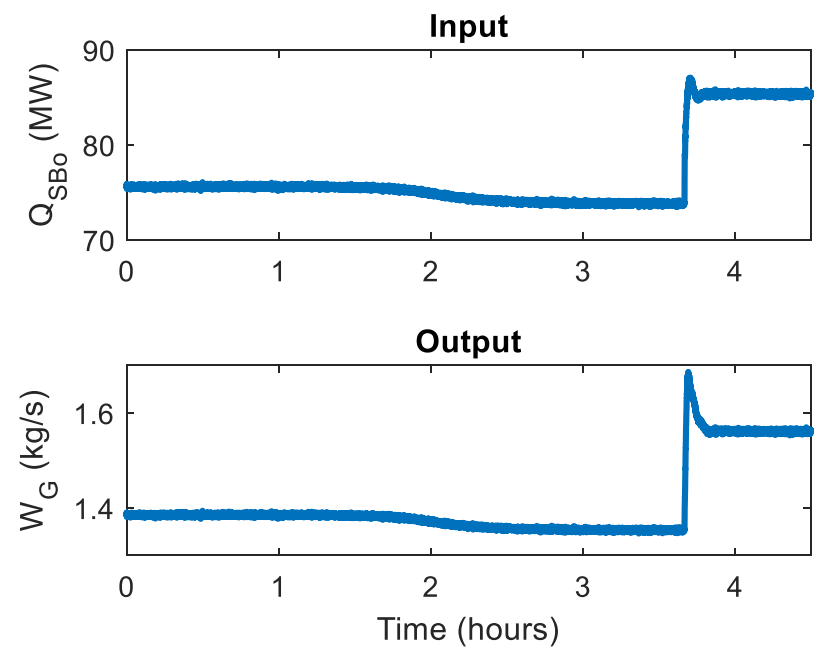

Figure 5-13. Dynamic response of $\mathrm{W}_{\mathrm{G}}$ for changes in $\mathrm{Q}_{\mathrm{SBo}}$.

Note that although the black-box equations of the model have been obtained, still, the first-principles equations parameters must be found to complete the model. To do so, following the methodology presented in Section 3.2, the black-box equations obtained must be added to the first-principles equations, and a least-squares constrained regression problem must be solved. However, considering that our plant is simulated, we already know the actual values, which can be found in Appendix A, and for the sake of briefness, we have skipped directly to the validation section.

\subsection{Validation}

To carry on with the validation step, first, we need to make a symbolic manipulation of the model obtained in order to simulate it. To do so, we have used Ecosimpro ${ }^{\circledR}$ as modeling and simulation environment, which supports acausal equations, giving more freedom to the modeler. Thus, after introducing the equations in any order and the boundary variables, the program itself finds the model causality, alerting about possible algebraic or high-index problems that can be treated with a setup assistant (Cellier, 1991). Table 5-1 shows the principal features of the resulting optimization model and compares them with the simulator model used as real plant. 
Table 5-1. Main features of the optimization and simulator models.

\begin{tabular}{c|c|c}
\hline & $\begin{array}{c}\text { Simulator } \\
\text { model }\end{array}$ & $\begin{array}{c}\text { Optimization } \\
\text { model }\end{array}$ \\
\hline Number of equations & 6485 & 52 \\
\hline - Static & 6036 & 38 \\
- Dynamic & 449 & 14 \\
\hline Parameters & 2131 & 23 \\
\hline Variables & 6456 & 48 \\
\hline Inputs & 29 & 4 \\
\hline Non-linear algebraic loops & 8 & 3 \\
\hline
\end{tabular}

The size of the resulting dynamic model accomplishes the objectives searched in the modeling stage. It has four inputs $\left(W_{B S t O u t}, E_{G}, T_{S B o}, P_{S S a O u t}\right)$, the same as the real system, and a reduced number of variables and parameters, which makes it appropriate for our optimization purposes. Furthermore, to run the simulation, we need a good starting point, which as it was explained in section 3.2, can be obtained from the dynamic data reconciliation. With the optimization model ready for simulation, the inputs shown in Figure 5-14 were introduced to both the simulator and the optimization model.

Their response have been analyzed in two different ways: First, both responses were compared graphically in Figure 5-15; and later, the Root Mean Squared Error (RMSE) (see Equation (5.41)) was computed to show the error of the model numerically, which are shown in Table 5-2. This index is one of the most used in the literature and calculates the error of the model with respect to the measurements, weighing the farthest predictions.

$$
R M S E=\sqrt{\frac{1}{N} \sum_{i=1}^{N}\left(y_{i}-\hat{y}_{i}\right)^{2}}
$$


(A)

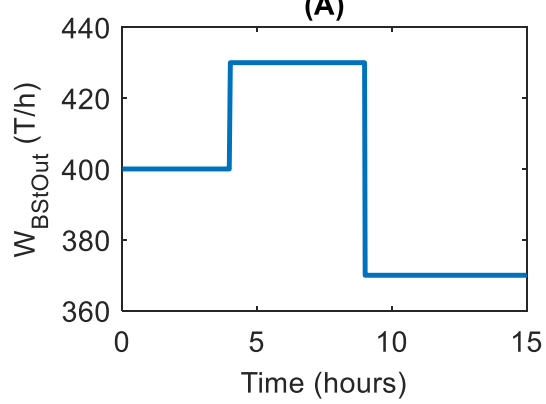

(C)

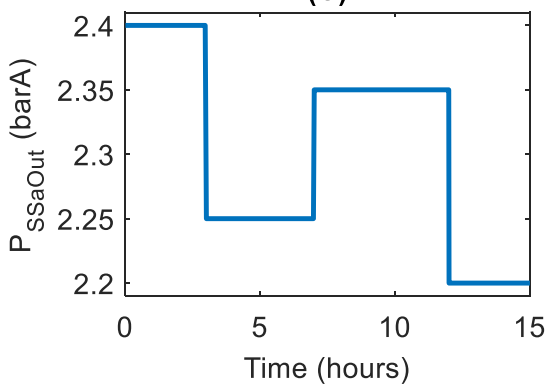

(B)

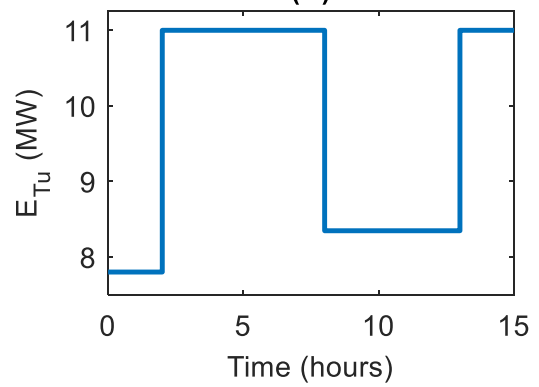

(D)

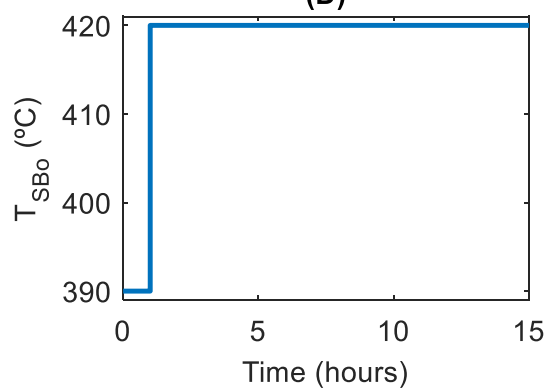

Figure 5-14. Input sequence for validation. (A) Beet processing rate $\left(W_{B S t O u t}\right)$; (B) Electricity power generation $\left(E_{T u}\right) ;(C)$ Evaporation working pressure $\left(P_{S S a O u t}\right)$; (D) Superheated steam temperature obtained in boilers $\left(T_{S B o}\right)$.

Table 5-2. Analytical model validation results

\begin{tabular}{c|c|c|c}
\hline Output Variable & RMSE [Ud] & Output Mean [Ud] & Relative Error [\%] \\
\hline$Q_{p}$ & $0.566 \mathrm{MW}$ & $67.61 \mathrm{MW}$ & 0.84 \\
\hline$E_{p}$ & $0.037 \mathrm{MW}$ & $7.79 \mathrm{MW}$ & 0.47 \\
\hline$W_{G}$ & $0.018 \mathrm{~kg} / \mathrm{s}$ & $1.47 \mathrm{~kg} / \mathrm{s}$ & 1.24 \\
\hline$\tau_{S t}$ & $0.52 \mathrm{~h}$ & $45.96 \mathrm{~h}$ & 1.12 \\
\hline$P E S$ & 0.01 & 0.13 & 6.31 \\
\hline$P_{I V}$ & 0.01 barA & 1.22 barA & 0.52 \\
\hline
\end{tabular}


(A)

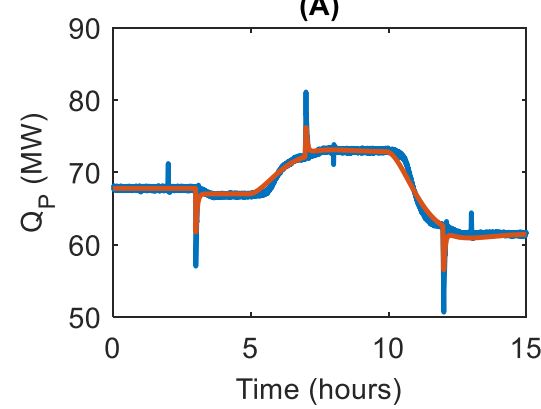

(C)

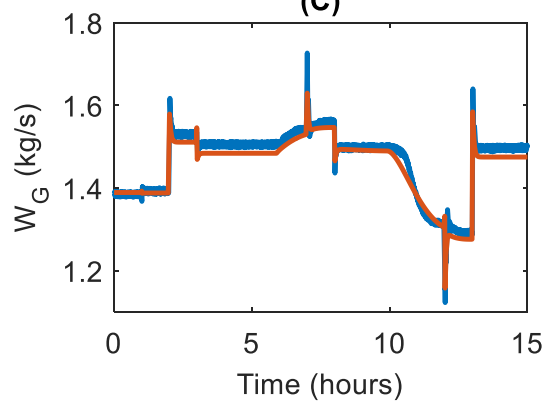

(E)

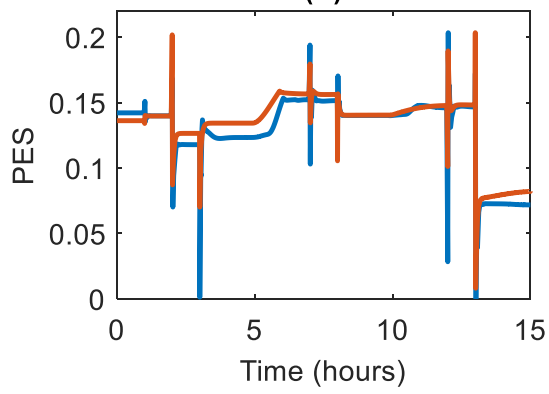

(B)

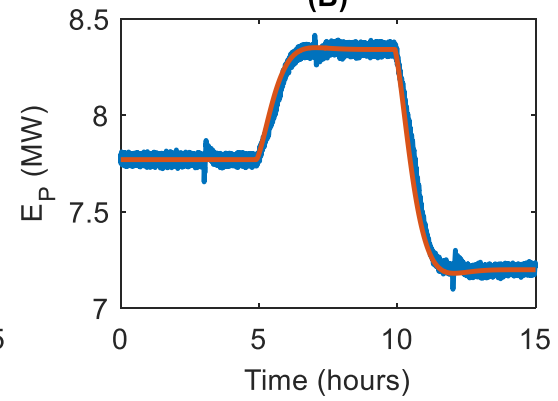

(D)

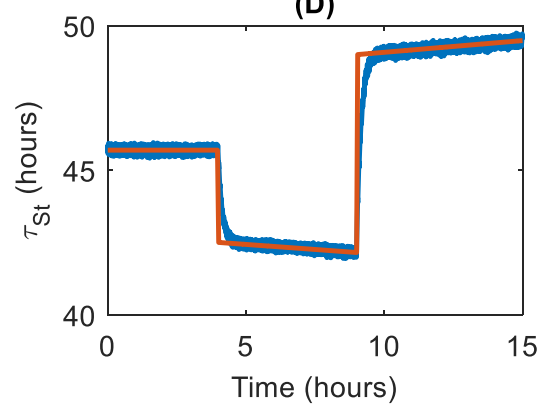

(F)

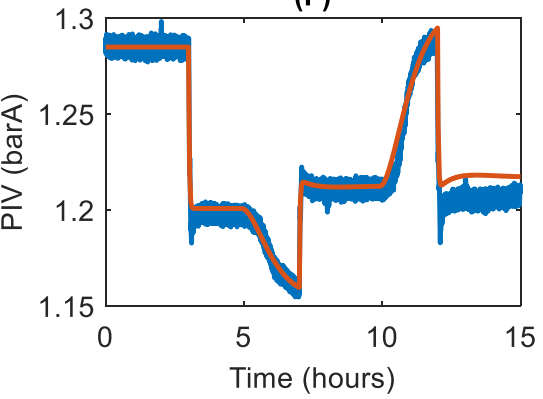

Figure 5-15. Outputs from data validation (blue simulator, orange optimization model).

(A) Heat energy consumption $\left(Q_{p}\right)$; (B) Electricity consumption $\left(E_{p}\right)$; (C) Natural gas mass flow rate; (D) Beet residence time stocked $\left(\tau_{\text {St }}\right)$; $(E)$ PES index; $(F)$ Steam pressure inside the evaporation fourth effect.

\section{Results discussion}

Figure 5-15 shows that, for almost all the time, the output of the optimization model follows the shape of the output of the simulator. For every output variable, it can be seen how the stationary state is captured reasonably well. The transient state is clearly affected by the linear assumptions made when obtaining 
the model. Here, the behavior of the model during slow and quick transients can be differentiated. For slow transients, caused by changes in production, the dynamic of the real plant is approximated by a straight line. This can be observed, for example, in $W_{G}$ from 10 to $13 \mathrm{~h}$ approximately. Although this response is not perfect, for the purpose of the model, it is sufficiently close to the real value of the plant. On the other hand, when quick transients are considered, in most cases, the model can predict such changes, but that fails when computing the amplitude. This is especially relevant in the prediction of the PES. However, since those transients are too short and this index is evaluated yearly, it is enough for the desired purpose.

The graphic results can be corroborated in Table 5-2. Here, for every variable except PES, the relative error is below $2 \%$. This means that the response of the model is very close to the response of the simulated plant. In the case of the PES, the quick transients, and a bias from hour 3 to hour 7 , make the relative error move to 6.31 . Traditionally, an error below $5 \%$ is considered sufficiently good. In our case, this value is slightly exceeded because of the reasons stated above.

With these results, we can conclude that the model accuracy is good enough to substitute the simulation module in the operational range considered and use it inside the optimization. This is key in order to use dynamic optimization with an industrial approach. The big number of dynamic equations joined to several hundreds of parameters needed make the simulation module not suitable for industrial dynamic optimization, since it would involve enormous computational times which would make difficult to perform tests and to obtain and maintain such model. 


\section{Chapter 6}

\section{Optimal operation of a beet-sugar factory working with a CHP unit}

In this chapter we address the formulation of the dynamic-integrated optimization problem applied to the simulated sugar factory case study depicted in Chapter 4. Such work has been done based on the model of the system previously obtained in Chapter 5.1. To solve the resulting non-linear dynamic optimization problem, following the methodology described in Chapter 3, we will use a simultaneous approach with a gradient-based solver like IPOPT. All the model equations will be discretized using orthogonal collocation, so no external simulator will be needed.

The chapter is structured in the following way. In the first section, we present the formulation of the optimization problem solved. Since part of the formulation has already been presented in the previous chapter, first, we focus on the reformulation of part of such model to make it suitable for using a gradient-based solver. Later, some specific constraints that deal with the legislation applied and the electricity market are explained, and finally, the objective function is presented. Considering that weights must be applied in the different terms of the objective function, once the whole optimization model is defined, the tuning of such parameters is addressed in the second section. Lastly, 
in the third section of this chapter, the results of the optimization are shown in two different ways. First, the optimizer response is analyzed for some isolated days to analyze in detail the tool performance. Later, the results for a whole campaign are presented and discussed.

\subsection{Optimization problem formulation}

\subsubsection{Model reformulation}

As it was mentioned before, note the reader that the strategy that will be followed to solve the dynamic optimization problem is the simultaneous approach using a gradient-based solver. It is well known that discontinuous equations must be avoided when using this kind of algorithms, however, sometimes such discontinuous expressions cannot be avoided, and must be addressed somehow. In the following subsections we will address the solution adopted to deal with discontinuities in several points of the process.

\section{Split-range Controller}

In the previous chapter the split-range controller was modeled using max and min clauses (Equations (5.10) and (5.11)). This was done to simulate the discontinuous behavior of the controller, but now a reformulation is needed. To introduce such equations in the optimization problem, complementarity constraints have been used, where complementarity refers to the relationship between variables where one (or either both) must be at its bound. More information about them can be found in (Biegler, 2010).

$$
\begin{gathered}
O p_{B y}(t)=\frac{v_{\max }}{v_{\max }-45} \cdot(v(t)-45) \cdot y(t) \\
O p_{R e}(t)=\frac{-v_{\max }}{45} \cdot(v(t)-45) \cdot(1-y(t)) \\
45-v(t)=S_{0}-S_{1}
\end{gathered}
$$

where:

$$
0 \leq y \perp S_{0} \geq 0
$$




$$
\begin{gathered}
0 \leq(1-y) \perp S_{1} \geq 0 \\
S_{0}(t) \geq 0, \quad S_{1}(t) \geq 0, \quad 0 \leq y(t) \leq 1 \\
0 \leq O p_{B y}(t) \leq 100, \quad 0 \leq O p_{R e}(t) \leq 100
\end{gathered}
$$

and $\perp$ is the complementarity operator, enforcing at least one of the bounds to be active. In (Biegler, 2010), four different formulations for the complementarity conditions were compared, and whether they should be implemented as constraints or as additional terms in the objective function was discussed. The results in that work showed that the best way to deal with them is using a penalty term in the objective function and solving the problem for a penalty parameter $\rho$. Therefore, this approach has been followed, and the implementation of these conditions will be addressed in the objective function section.

\section{Storage zone}

In the previous chapter, the storage zone was modeled using equations (5.5) and (5.6). With such equations, respectively, the beet residence time $\left(\tau_{S t}\right)$ and the mass accumulation in the storage zone $\left(m_{S t}\right)$ were computed. Now, for the optimization problem, we define two new variables to estimate if the residence time exceeds or falls below some limits given by the process knowledge. The upper bound has been established in 120 hours to avoid sugar losses because of potential beet degradation. The lower bound has been set in 15 hours to protect the process from running out of beet. In the objective function, these variables will be used to penalize undesired behaviors.

$$
\begin{aligned}
& \operatorname{StoPenUp}(t)=\max \left(\tau_{S t}(t)-120,0\right) \\
& \operatorname{StoPenLo}(t)=\max \left(15-\tau_{S t}(t), 0\right)
\end{aligned}
$$

Analogous to equations (5.10) and (5.11), since they involve the use of max and min terms, they have been reformulated using complementarity conditions, which will be enforced including an extra penalty in the objective function: 


$$
\begin{gathered}
\operatorname{StoPenUp}(t)=\tau_{S t}(t)-120+S_{3}(t) \\
\operatorname{StoPenLo}(t)=15-\tau_{S t}(t)+S_{5}(t) \\
\tau_{S t}(t)-120=S_{2}(t)-S_{3}(t) \\
15-\tau_{S t}(t)=S_{4}(t)-S_{5}(t)
\end{gathered}
$$

where:

$$
\begin{gathered}
0 \leq S_{2}(t) \perp S_{3}(t) \geq 0 \\
0 \leq S_{4}(t) \perp S_{5}(t) \geq 0 \\
S_{2}(t) \geq 0, \quad S_{3}(t) \geq 0, \quad S_{4}(t) \geq 0, \quad S_{5}(t) \geq 0
\end{gathered}
$$

\subsubsection{Specific constraints}

In this section, first, we will explain the formulation of the legislation referred to the efficient use of cogeneration systems in our optimization problem. Later, we will consider the interaction between the beet-sugar factory and the external grid. While the system model is process dependent, these constraints must always appear adapted for each case study.

Directive 2012/27/UE: Application

As it was explained in Section 4.3, to measure the efficiency of cogeneration processes, we recall that Directive 2012/27/UE defines that any cogeneration system with an installed capacity of more than $1 \mathrm{MWe}$ is considered as highly efficient if the Primary Energy Savings (see Equation (4.1)) are at least $10 \%$ with respect to the separate generation. The PES index is evaluated yearly, so its enforcement within optimization routines with shorter prediction horizons can be a challenge. To make sure the legislation is respected, the index is calculated for each instant $(t)$, and the integral of the index throughout the prediction horizon must be greater than the minimum accepted value (0.10) times the 
prediction horizon. Thus, the optimizer has freedom to find solutions which can eventually go below the lower bound.

$$
\int_{0}^{T} P E S(t) d t \geq 0.1 \cdot T
$$

According to Directive 2012/27/UE, a cogeneration plant can be divided into two different parts, CHP and non-CHP. For the case studied, if the global efficiency of the plant $\left(\mu_{G}\right)$ is equal to or greater than $75 \%$, then it is considered that all the fuel, heat, and electrical energy is generated in the CHP part (see Equation (4.4)). However, if the efficiency goes below $75 \%$, then part of the fuel and electrical energy is obtained in the non-CHP part. Again, the global efficiency of the plant is evaluated annually so, in order to ensure that this efficiency is always above $75 \%$, the same approach carried out for the PES index has been used.

$$
\int_{0}^{T} \mu_{G}(t) d t \geq 0.75 \cdot T
$$

\section{Electricity market}

For the electricity market, it has been assumed that the electricity bids sent to the market operator are always accepted. This assumption has been done based on the idea of the retailer bidding for the electricity needed or the remnant with a sufficiently low price with respect to the expected matching value (or even zero). Since the electricity market is marginalist, the final market price will be the same for every agent, and the price paid or obtained for the electricity negotiated will depend on the tariff used and the exact conditions negotiated. Therefore, in this work, three different possibilities have been considered. Two are based on tariffs with fixed prices, and the last one is directly based on the market electricity prices. These options cannot be combined, so only one of them will be used at a time:

- $\quad$ Base Load Contract (BL)

- Time of Use (TOU)

- Day-Ahead Market 
One of the main features of the optimization problem presented in this work is its capability to calculate the amount of electricity that the intermediary should bid on in the market based on the predictions made regarding the electricity price and beet availability. The electricity generated in the turbines must always be equal to the beet-sugar process demand $\left(E_{p}\right)$ plus the power committed with the external grid $\left(E_{C}\right)$, which considering that the market bids are hourly based, must always remain constant within an hour:

$$
E_{T u}(t)=E_{p}(t)+E_{c}(t)
$$

Considering the big process inertia, and the sharpness of changes in the electricity commitment, the split-range pressure control system is used to absorb the oscillations in the steam generation caused by the perfect matching of electricity generation and demand. Note the reader that in the simulation model, the electricity committed to the external grid was not considered and the power generated in turbines was a boundary value. In the optimization problem, with Equation (6.12), $E_{T u}$ is computed from $E_{C}$, which is a decision variable, and $E_{p}$, which is computed using Equation (5.3).

\subsubsection{Objective function}

In the objective function, apart from the specific energy cost, which was defined in Equation (5.1), four more different terms have been added with diverse aims. Each one of them is fully explained next.

$$
\begin{gathered}
J:=\text { Specific Cost }+ \text { Complementarity term }+ \text { Moving Cost } \\
+ \text { Storage Penalty }+ \text { Turnpike Cost }
\end{gathered}
$$

\section{Complementarity term}

In order to model the split-range controller and the storage penalties, complementarity conditions have been used, as previously described. Hence, to implement such constraints in the optimization problem, a penalty term has been added to the cost function. Thus, the problem feasibility is not compromised, avoiding the inclusion of tight equality constraints into the 
optimization problem. Therefore, two different terms have been added, each one with a different weight $\rho$.

$$
\begin{gathered}
\text { Complementarity term }= \\
\frac{1}{T} \int_{0}^{T}\left(\rho_{S R}\left(y(t) \cdot S_{0}(t)+(1-y(t)) \cdot S_{1}(t)\right)\right. \\
\left.+\rho_{S P}\left(S_{2}(t) \cdot S_{3}(t)+S_{4}(t) \cdot S_{5}(t)\right)\right) d t
\end{gathered}
$$

\section{Smoothing Term}

To avoid high frequency oscillations in the solution, a penalty term on the variation of the decision variables is added to the objective function. The weights of this term must be used carefully, since very high values deeply affect the original solution given by the optimizer.

$$
\begin{gathered}
\text { Moving Cost }= \\
\frac{1}{T} \int_{0}^{T}\left(\rho_{W_{\text {BStOut }}}\left(W_{\text {BStOut }}(t)-W_{\text {BStOut }}(t-\Delta \mathrm{t})\right)^{2}\right. \\
+\rho_{T_{S B o}}\left(T_{S B o}(t)-T_{S B o}(t-\Delta \mathrm{t})\right)^{2} \\
\left.+\rho_{\text {PSaOut }}\left(P_{\text {SSaOut }}(t)-P_{\text {SSaOut }}(t-\Delta \mathrm{t})\right)^{2}\right) d t
\end{gathered}
$$

Where $t$ is the instant when the control input is evaluated and $t-\Delta \mathrm{t}$ is the current instant minus a preselected time-step.

\section{Storage Penalty}

This term is added to penalize values of the beet residence time outside the preestablished bounds. Remember that with equations (6.6) - (6.7), high or low residence time $\left(\tau_{S t}\right)$ is calculated for each time $t$. Then, the integral of that term is computed, so the longer the residence time is out of bounds, the higher the penalty applied, allowing a small violation of the limits if necessary.

$$
\text { Storage Penalty }=\rho_{S t} \int_{0}^{T}(\operatorname{StoPenUp}(t)+\operatorname{StoPenLo}(t)) d t
$$




\section{Turnpike cost}

The turnpike property (Dorfman et al., 1958; Ellis et al., 2014; Faulwasser et al., 2017) is used to describe the response that appears in many finite-horizon dynamic optimization problems, where the solution is forced to pass through the optimal steady-state before reaching the final point, which gives a better economic result. This behavior appears when the prediction horizon is large enough, so the optimizer "pays" the cost of the turnpike (the optimum steadystate) to reach the final point with the least possible cost. Thus, if the predicted trajectory stayed at the final point for too long, the overall cost would be higher than passing through the optimal steady-state point for some time.

In this case study, the prediction horizon is one day, which is long enough to let the turn pike property appear and, at the end of the predicted state trajectory, the optimizer moves the plant away from the optimum steady-state point. The problem with this behavior is that the optimizer does not know that another optimization will be run the next day, so it moves the plant to a point that is hardly convenient to be the starting point for that day. To deal with this problem, two complementary strategies are proposed. The first one aims to assure that the final point suggested by the optimizer is a stationary point. Thus, the solution can be bad, but at least it is not a transient to an unknown destination. The implementation has been performed using terminal constraints on the end state, which ensure that the gradient w.r.t the decision variables is zero. Next, the steady state achieved is desired to be the best possible one. To do so, a final term is added to the objective function which represents the cost of maintaining the process at such a state during the first five hours of the next day, assuming the electricity price and beet arrival to be the ones already used for the first five hours of the current day. Five hours have been used because that is the slowest plant dynamics $\left(\tau_{\min }\right)$.

$$
\text { Turnpike Cost }=\rho_{T p} \int_{0}^{\tau_{\min }}\left(\frac{\frac{W_{G}(T)}{d_{G}} \cdot H H V_{G} \cdot P r_{G}-\frac{E_{C}(T)}{3600} \cdot P r_{e}(t)}{W_{B S t o u t}(T)}\right) d t
$$




\subsection{Penalty weights tuning}

Note that a good selection of the penalty weights for $(6.14)-(6.17)$ is essential for the performance of the described methodology. A wrong choice could lead to suboptimal or infeasible solutions. This is critical in the case of the complementarity terms since, otherwise, the split range would not be correctly modeled, and the compliance of the storage penalty could not be ensured. In Section 3.3, we described a methodology to find such values, and in this section, we apply it to our case study. In Appendix A the selected values for our case study can be found.

The first stage of the methodology consists in selecting representative scenarios that cover the full operation spectrum for one operation day. The number of representative scenarios that must be chosen depends on the number of exogeneous input variables. In our case, we have 2 exogeneous variables, the electricity price and the beet arrival, so we need four scenarios, which will correspond to extreme cases:

1. High electricity price and low beet arrival (PHBL).

2. High electricity price and high beet arrival (PHBH).

3. Low electricity price and low beet arrival (PLBL).

4. Low electricity price and high beet arrival (PLBH).
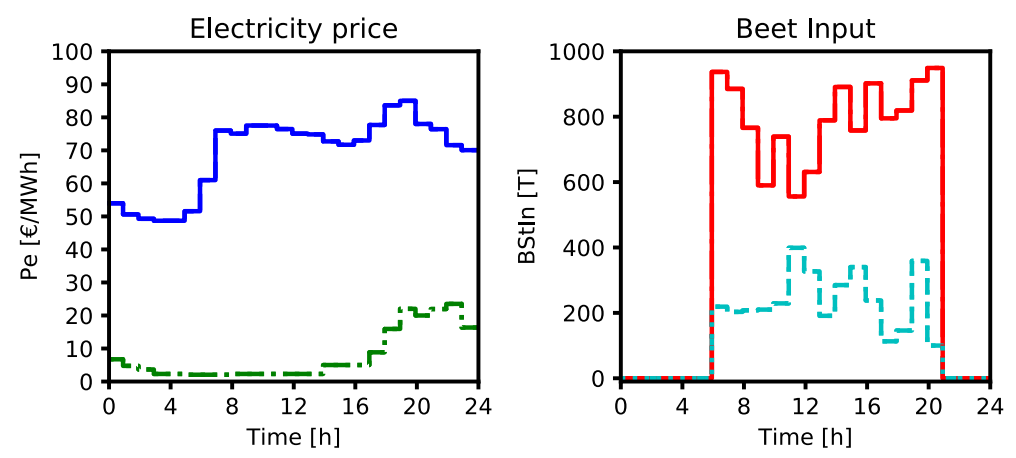

Figure 6-1. Values selected for the exogeneous input variables of our model. High electricity price (solid blue); Low electricity price (dashed-dotted green); High beet arrival (solid red); Low beet arrival (dashed cyan). 
Now, we need to find the minimum penalty weight that affects the solution, which is the supreme of the values found for each scenario, and then, increase it to cope with the design preferences. Note the reader, that for our case study, this task has been performed in a sequential way, that is, starting with the most critical weight, we set to 0 the rest of the weights, we obtained a tuned value, we fixed it, and then we followed with the rest of the penalties. The followed order is shown below:

1) Complementarity split-range controller term $\left(\rho_{S R}\right)$.

2) Turnpike term $\left(\rho_{T P}\right)$.

3) Smoothing terms $\left(\rho_{W_{\text {BStout }}}, \rho_{T S B o}, \rho_{\text {PSSaOut }}\right)$.

4) Complementarity storage penalty term $\left(\rho_{S P}\right)$.

5) Storage penalty $\left(\rho_{S t}\right)$.

For such a task, following the methodology, we have used the bisection method, and to evaluate whether the solution is affected or not, for each penalty weight, we have looked if the values of the following outputs remained constant between iterations:

- $\rho_{S R}$ : Opening of the bypass and relief valves $\left(O p_{B y}, O p_{R e}\right)$.

- $\rho_{T p}$ : Decision variables at the end of the prediction horizon $\left(W_{S t u O u t}, T_{S B o}, P_{S S a O u t}, E_{T u}\right)$.

- $\rho_{W_{B S t u o u t}}$ : Beet mass flow leaving the storage zone $\left(W_{\text {Stuout }}\right)$.

- $\rho_{T_{S B o}}:$ Temperature of the steam leaving the boilers $\left(T_{S B o}\right)$.

- $\rho_{P_{\text {SSaOut }}}:$ Pressure of the steam leaving the saturator $\left(P_{\text {SSaOut }}\right)$.

- $\rho_{S P}$ : Computation of the variables StoPenUp and StoPenLo.

- $\rho_{S t}$ : Storage residence time $\left(\tau_{S t}\right)$.

We recall that for the complementarity penalty weights, an increment from the above minimum values may cause distortions in the problem geometry without potential benefits, so we have kept the weight equal to the minimum found. In Figure 6-2 and Figure 6-3 we show the influence of the penalty weights on the solution for the PHBH scenario. To keep it short, we only show the results 
for the turnpike penalty weight $\left(\rho_{T P}\right)$ and the beet storage output smoothing weight $\left(\rho_{W_{\text {BStOut }}}\right)$.

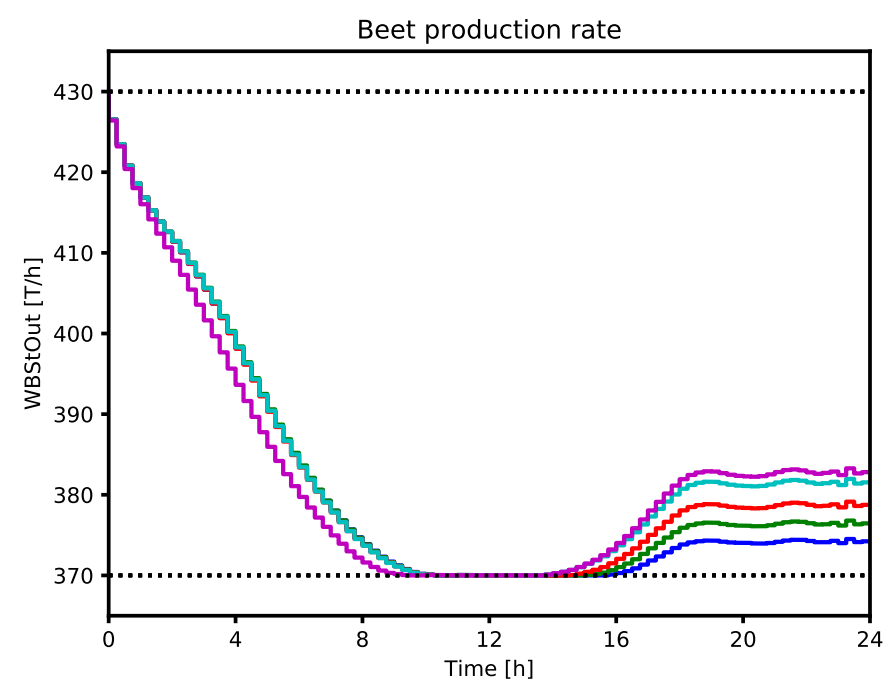

Figure 6-2. Beet production rate suggested by the optimizer in the PHBH scenario for different $\rho_{T P}$ values. In blue $\left(\rho_{T P}=0\right)$; In green $\left(\rho_{T P}=0.05\right)$; In red $\left(\rho_{T P}=0.1\right)$; In cyan $\left(\rho_{T P}=0.2\right)$; In magenta $\left(\rho_{T P}=1\right)$.

As Figure 6-2 shows, the beet processing rate can change significantly for the late hours of the day depending on the value of $\rho_{T P}$. We recall that to deal with the turnpike property we count with two different strategies. In Figure 6-2, we see that independently of the value of $\rho_{T P}$ the optimization end-point is always a stationary point. This was enforced with terminal constraints as explained in the previous section. Furthermore, we count with an expression that adds to the cost function the cost of maintaining the process in such steady state for five hours assuming that the electricity price and the beet input are the same as the day before. Such expression is the one multiplied by $\rho_{T P}$, and we see that if we increase the penalty weight value, the final stationary point changes. Of course, the behavior of the optimizer makes sense in the last hours of the prediction horizon. Considering that the electricity price of the early hours is reasonably lower than for the late hours, the optimizer suggests moving the production upwards in order to sell less electricity and produce more sugar in the early hours of the next day. The higher the penalty weight value is, the more importance the end-point has over the cost function, so the penalty weight must be chosen 
carefully to not affect in excess the rest of the solution. We can see that if the weight value is too high (magenta), the solution for the rest of the day starts to change significantly in order to benefit the last point. We can also observe that starting from a value of zero, if we increase just a little the penalty weight, the optimization end-point changes significantly. However, as we approach to $\rho_{T P}=$ 1 , the changes are smaller.

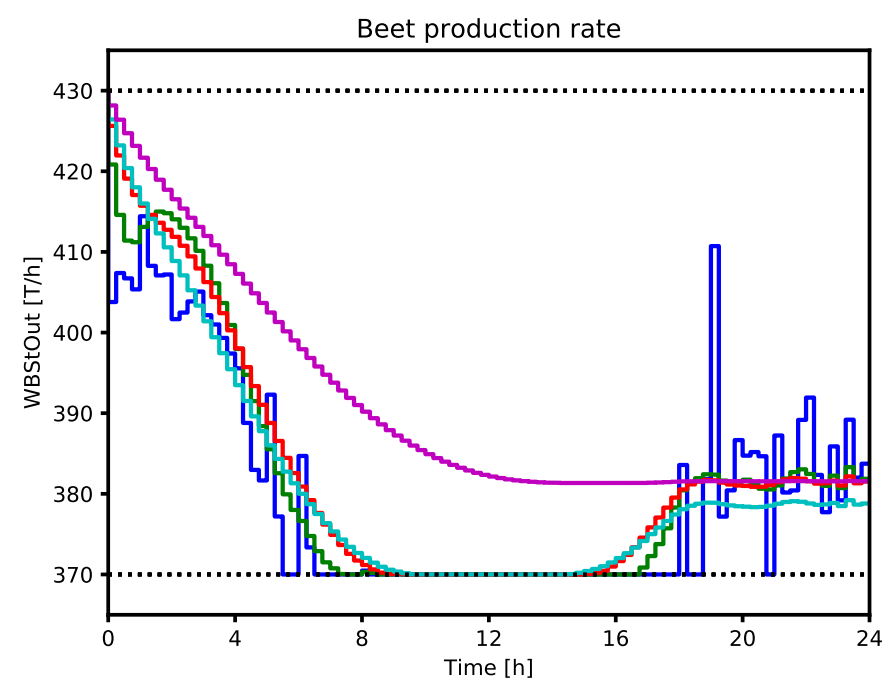

Figure 6-3. Beet production rate suggested by the optimizer in the $\mathrm{PHBH}$ scenario for different $\rho_{W B S t O u t}$ values. In blue $\left(\rho_{W B S t O u t}=0\right)$; In green $\left(\rho_{W B S t O u t}=0.1\right)$; In red $\left(\rho_{\text {WBStOut }}=0.5\right)$; In cyan $\left(\rho_{\text {WBStOut }}=1\right)$; In magenta $\left(\rho_{\text {WBStOut }}=5\right)$.

In Figure 6-3, we see the influence of $\rho_{W B S o o u t}$ over the beet production rate, when the rest of the weights are fixed at their nominal values. We recall that such penalty is part of the smoothing term added to the cost function. In this case, we see that for small values of the penalty weight, the solution is highly oscillating, which could never be applied to a real system. As we increase the penalty weight, we see how the solution starts to smoothen, however, if the penalty weight is too high (magenta), the penalty over movements in the beet production rate is too high with respect to the rest of the cost function, and the optimizer prefers to keep the process far from the lower limit bound, strategy that considering the convenient electricity prices at noon is clearly not optimal. In the extreme case, if the penalty value is increased a lot, the optimizer would suggest not moving the production rate at all. 
Once we had a set of penalty weight candidates, we performed a local sensitivity analysis to check whether small variations in the penalty weights found caused important effects on the optimization results. For this purpose, first, we evaluated the energy cost for each of the selected scenarios using the nominal penalty weight values. Then, we set a $1 \%$ increment for each candidate and each scenario independently, and we evaluated the energy cost again. Finally, we computed the sensitivity as the percentage ratio of the energy cost variation w.r.t the nominal value. The results obtained can be found in Table 6-1.

Table 6-1. Local sensitivity analysis results. The absolute values in the even columns are the energy costs, nominal and with penalties numerically disturbed, for each scenario.

\begin{tabular}{|c|c|c|c|c|c|c|c|c|}
\hline & PHBL (€) & $\begin{array}{c}\text { Sensitivity } \\
\text { (\%) }\end{array}$ & $\mathrm{PHBH}(€)$ & Sensitivity & $\begin{array}{c}P L B L \\
(€)\end{array}$ & $\begin{array}{c}\text { Sensitivity } \\
\text { (\%) }\end{array}$ & $P L B H(€)$ & $\begin{array}{c}\text { Sensitivity } \\
\text { (\%) }\end{array}$ \\
\hline Nominal & 11668,43 & - & 12026,69 & - & 15106,35 & - & 16776,30 & - \\
\hline$\rho_{S R}$ & 11668,30 & $<0.01$ & 12027,84 & 0,01 & 15106,35 & $<0.01$ & 16776,30 & $<0.01$ \\
\hline$\rho_{S P}$ & 11668,43 & $<0.01$ & 12025,70 & $-0,01$ & 15106,35 & $<0.01$ & 16775,15 & $-0,01$ \\
\hline$\rho_{W_{B S t u O u t}}$ & 11668,43 & $<0.01$ & 12026,77 & $<0.01$ & 15106,35 & $<0.01$ & 16776,30 & $<0.01$ \\
\hline$\rho_{T_{S B o}}$ & 11668,43 & $<0.01$ & 12026,91 & $<0.01$ & 15106,35 & $<0.01$ & 16776,31 & $<0.01$ \\
\hline$\rho_{P_{\text {SSaOut }}}$ & 11668,43 & $<0.01$ & 12025,25 & $-0,01$ & 15106,35 & $<0.01$ & 16776,31 & $<0.01$ \\
\hline$\rho_{S t}$ & 11668,41 & $<0.01$ & 12025,74 & $-0,01$ & 15106,39 & $<0.01$ & 16775,59 & $<0.01$ \\
\hline$\rho_{T p}$ & 11668,07 & $<0.01$ & 12027,03 & $<0.01$ & 15106,35 & $<0.01$ & 16776,31 & $<0.01$ \\
\hline
\end{tabular}

\subsection{Results}

This section has been divided into two different parts. In the first subsection, the results obtained for a single-day operation are presented. These will be useful to analyze in detail the behavior of the optimizer for different scenarios, where the electricity prices and the beet input vary within usual ranges. In the second subsection, the results using the optimizer for a whole campaign are presented and compared to the scenario traditionally used in sugar factories, where the production rate is kept at its maximum, and the electricity excess is exported to the grid under a BL tariff with fixed prices. 


\subsubsection{Single-day response}

As mentioned before, in the considered case study, there are two exogenous parameters: the electricity price and the raw material received in the sugar factory (beet input). The price of the electricity is key to determine the convenience of selling or buying electricity from the external grid. Furthermore, monitoring the arrival of raw material is also essential, in order to maintain the beet residence time between limits, thus, minimizing the sugar losses and preventing the situation where no raw material is available for production.

To test the behavior of the optimizer, some scenarios with different electricity prices and beet inputs were selected. Different experiments were carried out by fixing either the price profile or the beet-input profile, in order to evaluate the optimizer solutions with respect to each non-influential factor. The starting point was the same for all the cases, which corresponds to the classical policy where production is carried out at the maximum rate in order to finish the campaign as soon as possible to reduce fixed expenses.
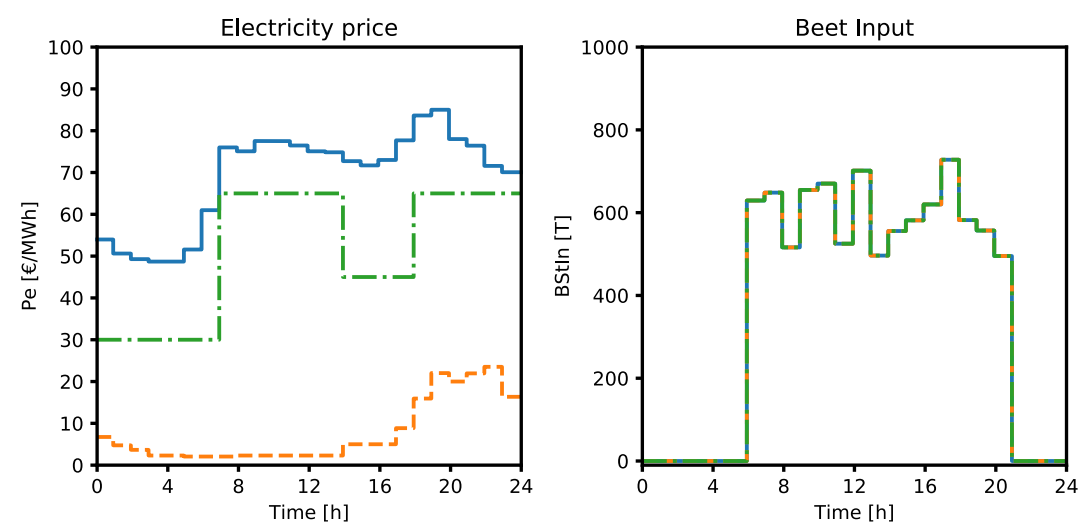

Figure 6-4. Scenarios used to test the response of the optimizer against different electricity prices. TOU rate (dashed-dotted green), Market high (solid blue) and Market low (dashed orange).

First, the beet input was fixed. As Figure 6-4 shows, trucks only deliver beet from 6 AM to 9 PM. The corresponding graph represents the total amount of beet received each hour. Three different electricity-price scenarios, obtained from the considered DR programs, were tested: 
- Time of Use tariff: with three different price zones $[30,45,65] € / \mathrm{MWh}$ depending on the hour.

- Spanish DA market prices for two different days: One with higher prices (10/12/2017), and another with much lower prices (30/12/2017).

Due to the large number of variables defined in the optimization problem, only the most important ones are shown here to present the results. Among them, the beet processing rate and the electricity commitment are key, of course. Moreover, as the way to deal with the legislation is one of the contributions of this work, the value of the Primary Energy Saving index is also presented. Yet, importantly, the policy followed to store the raw material is analyzed, given that a lack or an excess of stocked beet can invalidate the proposed management policies. Note the reader that to show the influence of the residence time boundaries in the solution, in this section we have changed the residence time upper limit to 30 hours instead of 120 hours presented in Section 6.1.1.
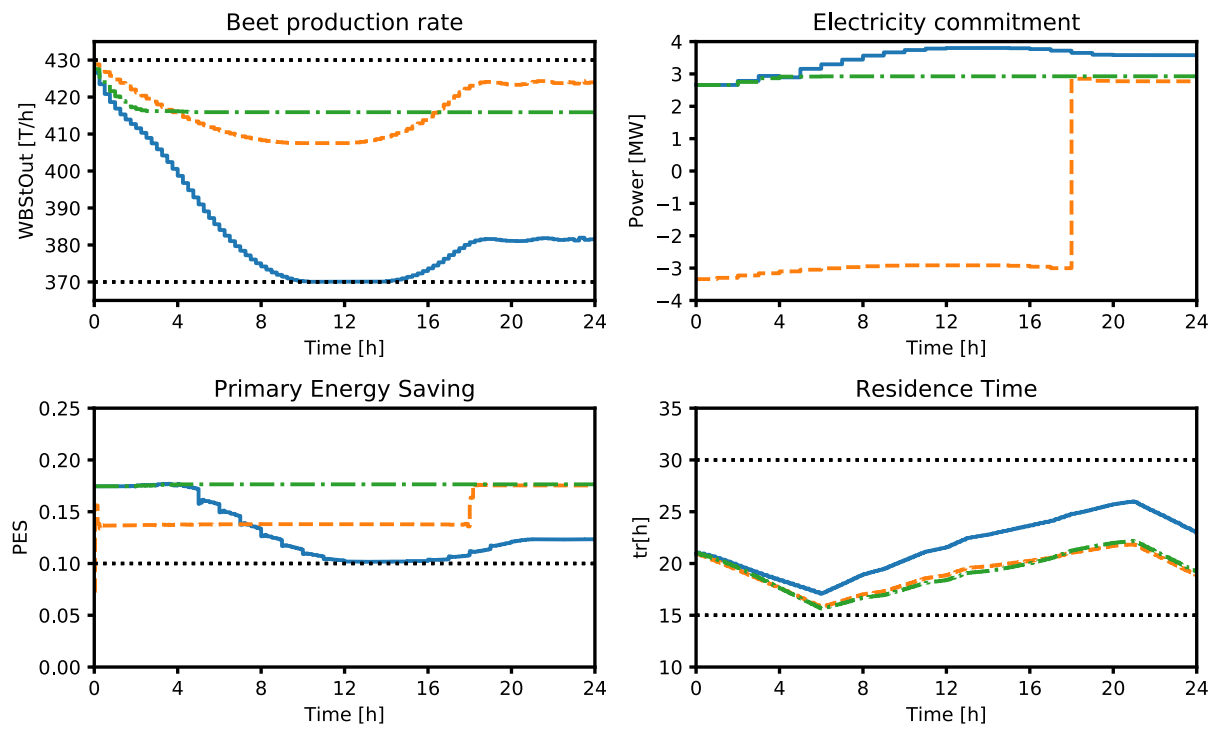

Figure 6-5. Summary of the results obtained for different electricity price scenarios. TOU (dashdot green), Market high (solid blue), Market low (dashed orange). 
From the results shown in Figure 6-5, it is observed that the computed response varies significantly, depending on the price policy. For example, if the market case with the highest electricity prices is taken, it can be noticed that the production rate goes for some hours to the minimum allowed. This is done to sell as much electricity as possible to the external grid (almost $4 \mathrm{MW}$ ) during that period, which makes sense considering its high price. Note that the PES value goes to the 0.1 limit from approximately the 10th until the 14th hour of the day; however, the integral of the index is above 0.10 , so equation (4.1) is not violated. Concerning the residence time, despite the aggressive policy suggested, it is easily kept between the bounds. From the 15th hour, the optimizer suggests increasing the production in order to be prepared for the next day. Recall the turnpike cost, which considers the five hours of the next day for the optimization final point using the electricity price and beet input of the current day. In this case the electricity price is considerably smaller for the first hours of the day, so the optimizer moves the process to an intermedium point.

In the lowest price scenario, the results are quite the opposite, and the production rate is maintained closer to its upper limit. Furthermore, the electricity price is so cheap that until the 18th hour the algorithm suggests importing power from the external grid and operate the boilers at a lower rate. Note that the beet processing rate is lowered until the 6th hour approximately, when new beet arrives to the plant (Figure 6-4), in order to keep the residence time above the lower limit of 15 hours. Then, production is kept nearly constant during some hours to regain stock for the next day, until, in the last hours, when enough stock is accumulated, the optimizer suggests increasing the production to an upper value. This analysis is key in order to understand that the optimizer suggests the highest possible production rate without violating the residence time constraint.

Regarding the TOU tariff, despite the price variations in the considered scenario, the optimizer suggests moving the plant to a stationary point. This can be justified considering that the process needs almost five hours to reach a steady state when operational changes are made in the production rate, so the algorithm needs the price to be maintained close to one point for several hours to consider the change of operating point worthwhile. This is observed in the market cases where the price is maintained high and low, respectively, almost the whole day. However, in the case of the TOU tariff, the price is only kept for a 
few hours and then suddenly changes, making the optimizer suggest an intermediate operational point. Nevertheless, note that the selected point is not just any random one, as it corresponds to the case where the bypass and relief valves are both closed, and the turbines are generating the maximum power. Intuitively, this is the point where less energy is wasted and, given that it can be considered as the optimum stationary operation from an energy point of view, the optimizer exploits it in several scenarios. From this operating point, if the optimizer suggested a lower production rate maintaining the electricity production, the relief valve would have to be opened and, if the production rate was suggested to be increased, the bypass valve would have to be opened, which is the case in the "market high" scenario.

In the second set of scenarios, the electricity price is fixed to the values of the "market high" scenario, and variations are made in the expected beet input. This price profile was selected to show the response of the optimizer when the electricity price is very convenient, but the beet storage is close to its limits. Again, all the tests started with the plant at the same operating point, and three different scenarios were considered (Figure 6-6):

- Standard input: the beet input profile is the same as in the previous test.

- High input: the input profile is higher because of the good weather conditions.

- Low input: due to adverse weather conditions, a small quantity of beet could be harvested and not many trucks arrived with raw material.
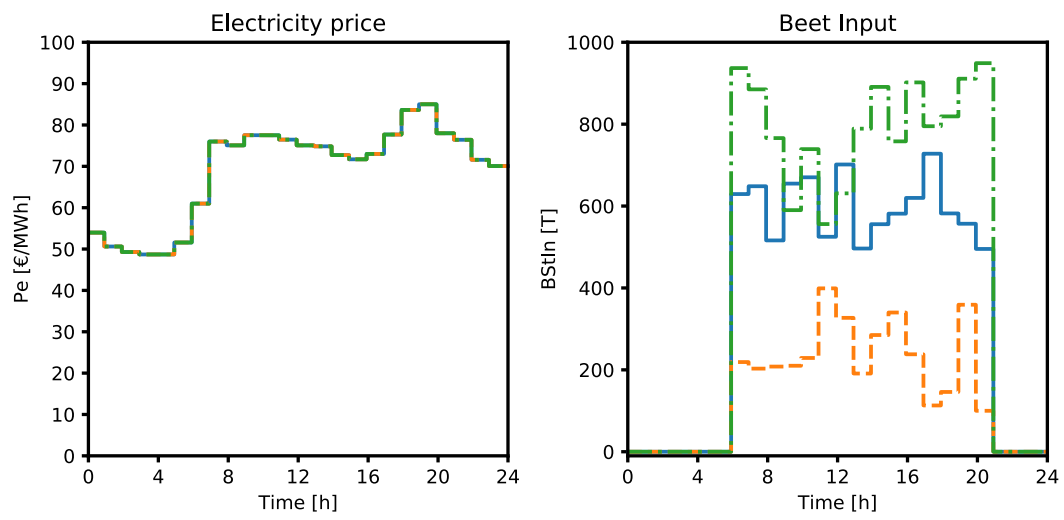

Figure 6-6. Scenarios used to test different beet inputs. Standard input (solid blue), High input (dashed-dotted green), Low input (dashed orange). 
The predicted optimal policies for these scenarios are shown in Figure 6-7. Again, the same variables have been selected to evaluate the solutions suggested by the optimizer.
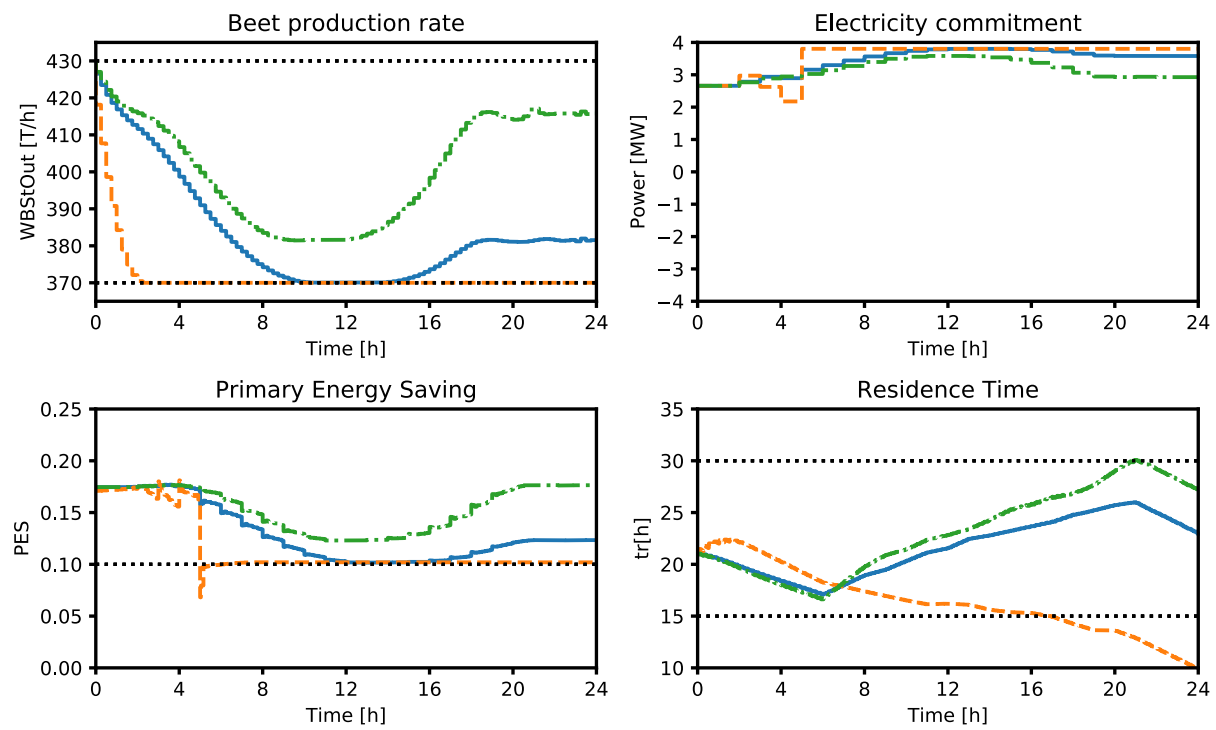

Figure 6-7. Results obtained for the beet-arrival test. Standard input (solid blue), High input (dashdot green), Low input (dashed orange).

From the results, several interesting conclusions can be extracted. Firstly, let us recall that the blue evolution in Figure 6-7 is the same as the response seen in the previous test for the market high scenario. This case serves as reference, since it is the free response of the optimizer when the residence-time constraints are not active. If we look at the high beet-input scenario, the reader can note how, despite the convenient electricity price, the production rate is much higher than for the nominal beet-input case. Of course, since less electricity is being sold, the cost function is penalized, but this is done to maintain the residence time just below its upper limit. It must be noticed in this case that, if only the electricity prices were considered, the storage area would be overwhelmed. In the opposite case, due to the low input of beet, the optimizer suggests going to the minimum operational point as fast as possible to keep the residence time above the lower limit. Despite all efforts, due to the extreme low input of beet, surpassing the minimum value set for the residence time cannot be avoided, but at least the least bad possible operation is suggested. 


\subsubsection{Multi-day response}

In this section we present and discuss some results for a multi-day scenario to show the response of the optimizer between days. We have considered five days using the Spanish market prices from 04/12/2017 to 08/12/2017. In Figure $6-8$, the electricity price and the beet-input range selected are shown.
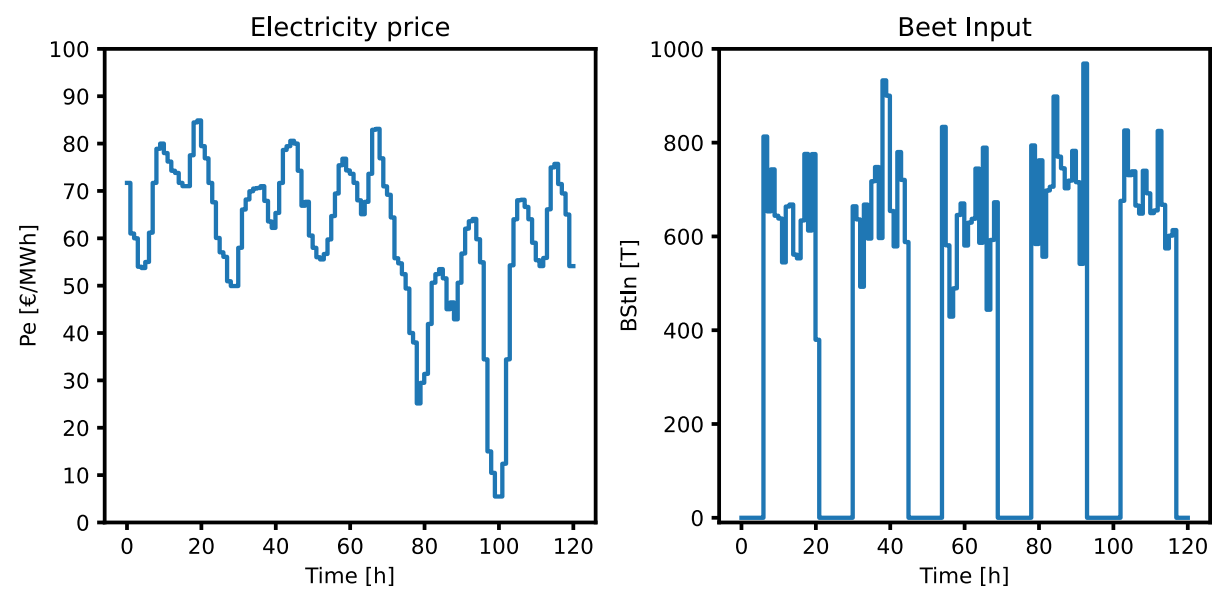

Figure 6-8. Electricity price and beet input for a simulated week.

From the results shown in Figure 6-9 and Figure 6-10, we can extract that when the electricity price is higher (the first 72 hours), the beet production rate is moved to a value close to the allowed lower bound of $370 \mathrm{t} / \mathrm{h}$. Thus, a higher excess of electricity can be generated, and more power is committed to the external grid. Nevertheless, the results are quite the opposite when the electricity price goes down, specially, around hour 100 , when the price is very close to $0 € / \mathrm{MWh}$. In such scenario, the optimizer suggests increasing the production considering that little benefit can be extracted from selling electricity. However, instead of establishing the operating point around the permitted upper bound, it moves the process to the optimum steady-state, seeing that for the rest of the hours the price is not cheap enough. Besides, looking at the electricity commitment chart, for the last $48 \mathrm{~h}$, we can observe that only for hour 100 , the optimizer decides buying electricity, while for the rest of the time it sells a smaller amount of electricity compared to the first 72 hours. In the rest of the charts, we can see that the constrained variables are always kept between bounds, and the evolution of some interesting variables. 

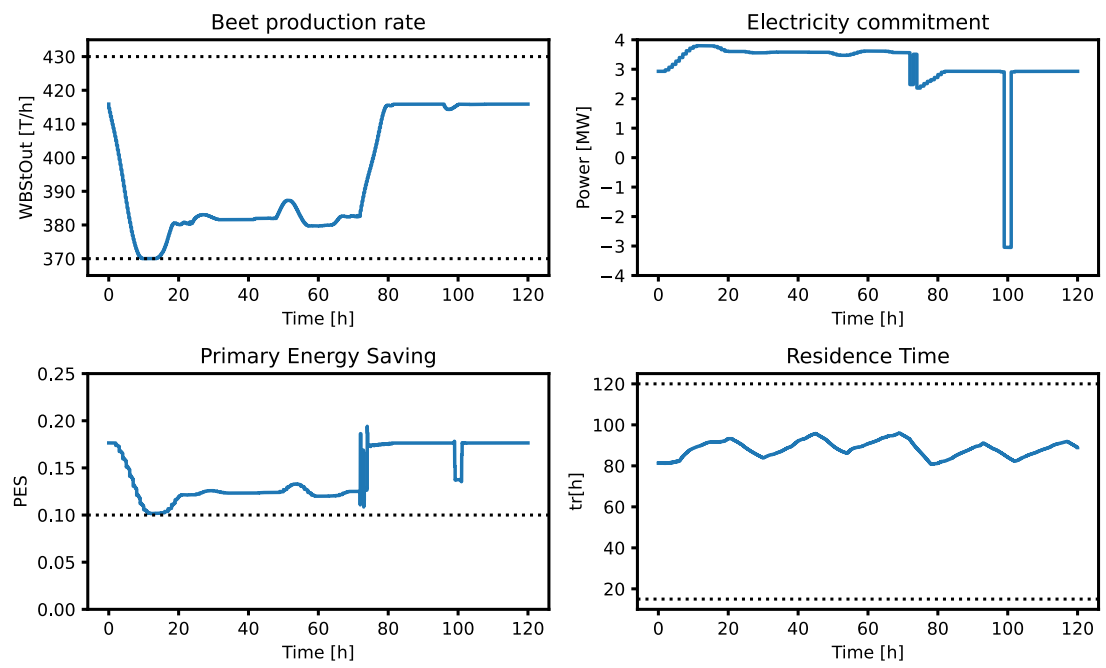

Figure 6-9. Results for some interesting variables obtained for a multi-day scenario (1)
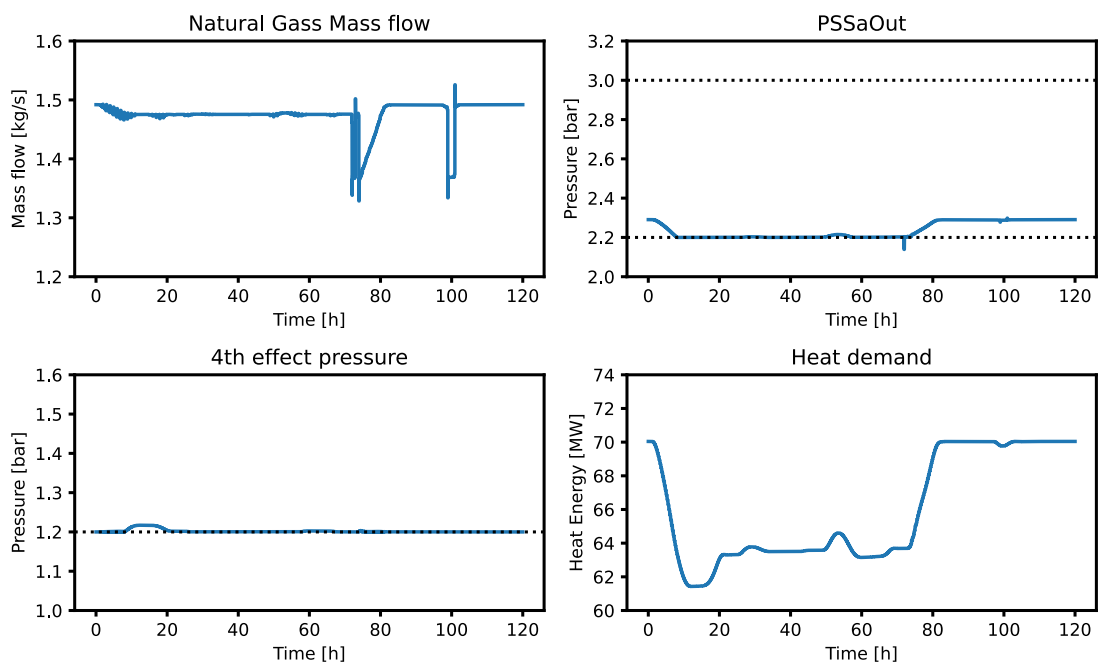

Figure 6-10. Results for some interesting variables obtained for a multi-day scenario (1) 


\subsubsection{Campaign results}

The sugar extraction is a seasonal business which, in the north of Spain, is typically carried out from approximately October to February. A common way of working is to fix the sugar production before starting the campaign, so in this work an objective of $138000 \mathrm{t}$ of sugar has been established. For an average polarization of $16^{\circ}$ and $90 \%$ of purity, it has been assumed that 959760 t of beet are necessary to obtain the sugar target. The minimization of the operating costs may lead to an extension of the campaign duration, and therefore, an increment in the fixed cost. Given that the difference between the minimum and maximum production rate in the factory is $1440 \mathrm{t} / \mathrm{d}$, for $959760 \mathrm{t}$ of beet, the maximum increase in the campaign duration can be 15 days. For this short period of time, it has been assumed that the rise of the fixed cost would come essentially from the extension of the workers' salary, which will be considered in the results.

First, a comparison between the different selected tariffs was carried out. This helped us to discern between the benefits provided by the optimizer suggested policy and the ones obtained by the tariff itself. To do so, the maximum rate strategy was applied using the following electricity rates:

- Base load: Beet processing rate is set to the maximum, and the electricity surplus generated is sold under a Base Load tariff with a fixed price of $50 € / M W h$ for the whole day.

- TOU: The electricity prices are set by a TOU tariff with three different price zones $[30,45,65] € / M W h$ depending on the hour.

- DA Market: The electricity prices are taken from the Spanish DA market, including from 30/09/2017 to 17/01/2018.

From the results shown in Table 6-2 we can conclude that both of the tariffs considered provide an important reduction in the energy cost without changing the operation strategy. It can be noted that the results show a commonly accepted trend, where the base load tariff gives the lowest gains with its conservative electricity prices, the TOU tariff gives intermediate results, and the spot market yields the greatest benefits at the expense of assuming a greater risk in the price. 
Table 6-2. Campaign results when the maximum rate strategy is used with the three different rates considered. The percentages show the difference with respect to the base load case.

\begin{tabular}{|c|c|}
\hline Tariff & Energy Cost (€) \\
\hline Base Load & $1,442,645$ \\
\hline TOU & $\begin{array}{c}1,433,989 \\
(-0.6 \%)\end{array}$ \\
\hline DA Market & $\begin{array}{c}1,400,086 \\
(-2.95 \%)\end{array}$ \\
\hline
\end{tabular}

Next, we gave freedom to the optimizer so it could extend the campaign a maximum of 5 days ( 120 hours). This is the upper limit that was stablished in the storage zone in Section 6.1.1, so the beet did not lose an important amount of sugar because of rotting or other problems. In this case, the energy cost could no longer be a representation of the overall cost of the campaign, and then, total expenses had to be considered to compare each strategy. For that purpose, it was considered that, since the maximum campaign extension was 5 days, the most important increase that the fixed costs may suffer came essentially from the salary that had to be paid to the workers during that period (as was previously indicated). During the campaign season in a traditional sugar factory of the size considered in this work, around 100 temporary workers are hired for the operation of the plant. If it is assumed that the company must pay $1710 € /$ month for each worker, corresponding to a typical net salary of $1000 € /$ month, the wage for the total temporary staff per day can be established at $5700 €$. Note that salaries for permanent personnel were not included in this computation, because these remain as fixed expenses. Therefore, in order to compare which strategy is the most convenient, the possible reduction in the energy cost is balanced with the extra salaries that should be paid with respect to ending the campaign in 94 days (the shortest possible).

Note the reader that our approach is implemented in a moving-window fashion with a prediction horizon of 1 day, and no rescheduling is performed during the whole operation. Apart from the energy cost, the following indicators are also presented to analyze the suitability of each solution: the campaign 
duration, the natural gas consumption, and the incomes provided by the electricity sale.

From Table 6-3, if we take a look to the results obtained for Case 1, where the TOU tariff is selected, we can observe that although the campaign duration has been increased in three days with respect to the base case, the energy cost including the salary increase has been reduced a $2.55 \%$. Of course, part of this increase is due to the change of tariff, that since we saw in Table 6-2 can be estimated as a $0.6 \%$, leaving the improvement due to the use of the optimizer in a $1.95 \%$. This has mainly been achieved by a reduction in the natural gas consumption $(0.21 \%)$, and an increase in the profit obtained by selling electricity to the external grid (16.45\%), which can be justified by the smarter use of the cogeneration system suggested by the optimizer, which is able to propose solutions where the energy is fully leveraged, as shown in the previous subsection.

Table 6-3. Campaign results with the upper storage constraint set to 5 days.

Percentages show the difference with respect to the base case.

\begin{tabular}{|c|c|c|c|c|}
\hline Tariff & $\begin{array}{c}\text { Energy cost + } \\
\text { salary inc. } \\
(\boldsymbol{\epsilon})\end{array}$ & $\begin{array}{c}\text { Campaign length } \\
\text { (d) }\end{array}$ & $\begin{array}{c}\text { Natural Gas } \\
\left(\mathbf{m}^{3}\right)\end{array}$ & $\begin{array}{c}\text { Electricity sale } \\
(\boldsymbol{\epsilon})\end{array}$ \\
\hline \multicolumn{5}{|c|}{ Base Case } \\
\hline Base Load & $1,442,645$ & 94 & $3,426,901$ & 300,009 \\
\hline \multicolumn{5}{|c|}{ Case 1 } \\
\hline TOU & $\begin{array}{c}1,406,698 \\
(-2.55 \%)\end{array}$ & $\begin{array}{c}97 \\
(+3.19 \%)\end{array}$ & $\begin{array}{c}3,419,649 \\
(-0.21 \%)\end{array}$ & $\begin{array}{c}349,366 \\
(+16.45 \%)\end{array}$ \\
\hline DA Market & $\begin{array}{c}\text { Case 2 } \\
\text { (-5.468,622 }\end{array}$ & $\begin{array}{c}97 \\
(+3.19 \%)\end{array}$ & $\begin{array}{c}3,416,265 \\
(-0.31 \%)\end{array}$ & $\begin{array}{c}385,722 \\
(+28.57 \%)\end{array}$ \\
\hline
\end{tabular}


However, the best results are obtained when the Day-Ahead tariff is used. In this case, again, the campaign length is extended by 3 days, and the energy cost is reduced by a $5.41 \%$ with respect to the base case. Considering a contribution of a $2.95 \%$ for the change of tariff, we conclude that approximately, the reduction due to the optimizer operation is a $2.46 \%$. Again, such results have been achieved thanks to a reduction in the natural gas consumption and an increase in the electricity profit obtained by selling electricity, which in this case is increased by a $28.57 \%$. This increase with respect to not only the base case, but also Case 1, can be explained considering the difference in the electricity price, and the more aggressive policy taken by the optimizer when the electricity prices are more convenient. We recall that for Case 1 , the solution given by the optimizer, which is shown in Figure 6-5, is always the same if the storage limits are not threatened, as the electricity price is always the same. However, for Case 2 , this is no longer true, and the optimizer changes its operation strategy each day depending on the electricity price and the beet stocked.

\subsubsection{Computational features}

In this case study, the optimization with a prediction horizon of a single day involves 25127 variables and 23671 equality constraints, which gives a total of 1456 degrees of freedom. To discretize the optimization model, the orthogonal collocation method (Biegler, 2010) was chosen, using Radau roots for collocation, and 97 finite elements with 3 collocation points per element. Thus, a total of 289 collocation points with a finite element of 15 -minutes in length was considered enough to obtain a good solution in a reasonable time, given the slow dynamics of the system.

The solver IPOPT was used in Pyomo 5.5.2 under Python 3.7.3 on an Intel i7$7700(3.60 \mathrm{GHz}) \mathrm{CPU}$, with $32 \mathrm{~GB}$ RAM and Windows 10 . The CPU time for solving this optimization varies greatly, depending on the scenario and the initial guess. The average CPU time in case 2 was $123.4 \mathrm{~s}$, the maximum being $1633 \mathrm{~s}$ corresponding to day 73 , and the minimum $15 \mathrm{~s}$ for day 17 . The optimizer must be run at least once per day before $10 \mathrm{AM}$. The electricity price and beet reception forecast must be introduced by the operator, so the optimizer computes the production and energy generation policy that must be followed during the next day. Hence, the computational time is not a limitation for the case study considered. 


\section{Chapter 7}

\section{Final conclusions and outlook}

\subsection{Contributions}

The achievement of the proposed objectives for this thesis has resulted in the following contributions:

- Study of the current situation and progress of the methodologies used to manage the production of industrial processes with and without cogeneration systems considering energy awareness. This topic has been covered in Chapter 2.

- Study of the Spanish electricity market and the current legislation concerning the efficient operation of cogeneration systems, and its application to a beet-sugar extraction process. All the details can be found in Sections 4.2 and 4.3.

- Methodology to obtain gray-box dynamic models to represent the closed-loop dynamic features of systems formed by industrial processes and cogeneration plants. Such methodology has been applied in silico to the case study considered. For more information, see Section 3.2 and Chapter 5.1 of this thesis. The results have been published in the journal Processes, which in the moment of publication was Q2 in the field of Chemical Engineering (Pablos et al., 2019). 
- Efficient formulation of the optimization problem using a dynamic approach based on the use of models representing the closed-loop dynamic features of the systems considered. Application to a simulated beet-sugar extraction plant under a Spanish legislative and economic framework. Study and comparison of the obtained results using two typical price-based demand response programs (TOU and Day-Ahead prices) with respect to operating with a base tariff and a non-energy awareness politic. The generic formulation of the problem can be found in Section 3.3, and its application in Chapter 6. The results have been published in the journal Computers And Chemical Engineering, which in the moment of publication was Q1 in the field of Chemical Engineering and Computer Science (Pablos et al., 2021).

- Software architecture concept to facilitate the industrial implementation of the proposed system based on the use of three interconnected modules. The architecture can be found in Section 3.4, and its application on the case study considered in Appendix B. The study of such implementation has resulted in several congress publications (Hernández et al., 2019; Pablos et al., 2017; Zamarreño et al., 2017).

\subsection{Conclusions}

In this thesis, we have developed a methodology to optimize the management of the production of industrial processes working with coupled cogeneration plants. Such methodology has been successfully applied to a simulated beetsugar extraction plant within a Spanish legislative and economic framework. The main conclusions drawn from this work, as well as some limitations that still need to be covered, are presented below:

- The formulation presented in this work is based on simultaneous dynamic optimization techniques that are able to provide results to the problem considered in short periods of time. This is positive not only from an implementation point of view, where results must be obtained faster than the frequency of use of the tool, but also from a usability point of view, since end-users will be more willing to use it if they do not have to wait for long time periods to obtain results. 
- The proposed methodology has been successfully tested in silico on an industrial process with a seasonal production, such as the beet-sugar extraction industry. Thus, despite increasing the duration of the campaign by 3 days, it has been found that operating costs can be reduced by $2.55 \%$ if the TOU tariff is used, and by $5.41 \%$ if the Day-Ahead tariff is used instead. These results reflect the fact that lower operating costs can be obtained assuming a higher risk in the electricity price. In any case, the results have always been better when compared to the case without energy awareness and constant maximum production.

- $\quad$ The proposed prototype software architecture presented in Section 3.4 and applied in Appendix B serves as an example to illustrate a possible industrial implementation. Of course, we are aware that many problems have been neglected due to the simulated nature of the system, but still, we have shown some of the complications that must be faced during the industrial implementation of a tool like the one developed and their solutions. In addition, we consider that being able to implement and update this and any other optimization tool in an easy way is vital for its approval in the industry. That is why we think that more attention should be paid to this issue, and studies of this kind should be carried out more frequently when new methodologies are proposed for industrial use.

- The methodology proposed in this thesis, like any other based on the use of price-based demand response programs, may not be valid in industrial processes where changes in the electricity price have a low impact on their economic results compared to other factors such as the raw material cost or the profits obtained from the processed products. Furthermore, we recall that it is only valid for industrial processes that meet the assumptions made in Chapter 3. Particularly relevant are the points concerning the dynamic features of the main process and the existing coupling between thermal and electrical energy. Note that, if the dominant dynamics of the main process are much faster than the changes in the electricity price, they could be neglected, and the optimization problem would be much simpler to solve. Furthermore, if there is no coupling, the operation of both systems could be treated independently, and sequential or iterative approaches would make more sense. Such decoupling could be achieved aggregating several types of technologies to the cogeneration unit (steam, gas, combined cycle...) or 
with the use of thermal and/or electrical energy storage systems. Both strategies have already been extensively studied in the literature.

- In this work we have studied the industrial application of the Spanish electricity market and the legislation associated with the efficient operation of cogeneration systems as generically as possible. However, the complexity in its application to each case study means that, although the bulk of the mathematical formulation of the constraints related can be directly extrapolated, certain details have to be adapted to each particular case.

- As a final conclusion, it should be noted that, although the results regarding the implementation of the developed methodology in the simulated case study have been satisfactory, we think that the seasonality of the process, as well as the beet storage policy, limit too much the degrees of freedom of the optimization problem and, therefore, the possible benefits of the methodology proposed. That is why, we think that better results could be obtained if this approach were used in other industries with higher flexibility in their production and storage policy.

\subsection{Open issues}

Currently, the development of decision support tools that lead to optimum energy-aware industrial production scheduling is becoming significantly important in the Process Systems Engineering community. Thus, the competitiveness of industrial process, and the stability of the power grid can be achieved at the same time. We have observed that to avoid possible suboptimal results if auxiliary generation systems are available, like cogeneration plants, it is important to consider the system as a whole, instead of a master-slave interaction. However, on many occasions, the high computational cost of this strategy when dynamic features are incorporated, limits its practical application and, therefore, future research should be aimed at reducing this cost, either by reducing the complexity of the mathematical formulation, or by improving the solvers efficiency.

Focusing on the optimization problem complexity, in this thesis we have successfully developed a methodology to efficiently formulate dynamic mathematical models of systems formed by industrial processes operating with 
on-site cogeneration plants. Such methodology can be extrapolated to a large number of cases in the process industry, but special attention must be paid when selecting the structure of the black-box part of the model, having to be adapted for each individual case. Future work can be directed towards new methodologies that helps to find the optimal structure of such black-box part for each individual case. In this direction (Cozad et al., 2015) and more recently, (Pitarch et al., 2019), proposed alternative ways for dealing with this semiinfinite programming problem. However, such methodologies are focused on static models, and its scalability is limited when the size of the problem increases, therefore, more work needs to be done in this field.

Furthermore, note that neither discrete decisions nor the effect of uncertainty has been included in the problem formulation. Of course, both features are present in the process industry in one way or another, but their implementation in dynamic optimization problems is still an open issue in the literature. Discrete decisions must be included if, for example, several products can be obtained simultaneously in the process plant considered, or if the unit commitment problem is considered in the cogeneration plant. The complexity lies in the formulation and solution of a mixed-integer dynamic optimization problem in a reasonable time and with certain optimality guarantees.

Regarding uncertainty, in the case study, it clearly appears in the electricity price and beet reception predictions. In order deal with it, a robust or stochastic approach can be used, but again, a high impact in the solution time is expected (Lucia, 2015). In addition, there is the structural uncertainty of the model (Navia López, 2013), which of course, is not perfect. In the literature, we can find several approaches that treat this problem, such as the two-step approach, modifier adaptation, or extremum seeking control among others (Rodríguez-Blanco, 2017). However, in our case, the fact that the process is not necessarily operating in steady state, as well as the dynamic nature of the model, will complicate its application and, therefore, a thorough research in this sense should be carried out in this field.

The future electricity markets can be expected as a challenge for our methodology. In the coming years, in Spain, historical differences are expected between the maximum and minimum prices with respect to the average daily price in the spot electricity market. This is due to the renewable policies applied on the generation side, which will imply: 
- An important increase in the solar photovoltaic installed capacity, which will reduce the electricity price in the central hours of the day.

- A boost in the price of $\mathrm{CO}_{2}$ emissions, which will make less profitable the carbon-based technologies, increasing the marginal price when less renewable energy is available.

In this scenario, our methodology, and all those related to price-based demand response programs aimed at flattening the demand curve by consuming more when the electricity price is cheaper, will stand out even more thanks to the bigger differences between the electricity price along the day.

Nevertheless, a higher volatility in the short-time electricity price is also expected due to the intermittency in the availability of renewable energy sources, which will affect, specially, to the intraday and the deviation management market, both managed during the dispatched day.

In this thesis, aware of the high inertia found in the industrial processes, we have based our study in the Day-Ahead electricity market, where the electricity price changes with a frequency that can be followed by such systems. Considering that in our approach decisions related to electricity sales are taken a day in advance, these changes in the final electricity price were not considered, but they could have been implemented as uncertainty in the final price, and, therefore, we propose it as future work. 


\section{References}

Acebes, L.F., de Prada, C., Gorostiaga, L., 1999. Evaluating cane feeding control. Int. Sugar J. (Cane Sugar Ed. 101, 495-500.

Acebes, L.F., Merino, A., Mazaeda, R., Alves, R., De Prada, C., 2011. Advanced dynamic simulators to train control room operators of sugar factories. Int. Sugar J. 113, 700-707.

Acebes, L.F., Merino, A., Rodriguez, A., Mazaeda, R., de Prada, C., 2019. Model based online scheduling of concurrent and equal batch process units: Sugar End industrial case study. J. Process Control 80, 1-14. https://doi.org/10.1016/j.jprocont.2019.05.005

Agha, M.H., Thery, R., Hetreux, G., Hait, A., Le Lann, J.M., 2010. Integrated production and utility system approach for optimizing industrial unit operations. Energy 35, 611-627. https://doi.org/10.1016/j.energy.2009.10.032

Aghaei, J., Alizadeh, M.I., 2013. Multi-objective self-scheduling of CHP (combined heat and power)-based microgrids considering demand response programs and ESSs (energy storage systems). Energy 55, 1044-1054. https://doi.org/10.1016/j.energy.2013.04.048

Albadi, M.H., El-Saadany, E.F., 2008. A summary of demand response in electricity markets. Electr. Power Syst. Res. 78, 1989-1996. https://doi.org/10.1016/j.epsr.2008.04.002

Alipour, M., Zare, K., Mohammadi-Ivatloo, B., 2016. Optimal risk-constrained participation of industrial cogeneration systems in the day-ahead energy markets. Renew. Sustain. Energy Rev. 60, 421-432. https://doi.org/10.1016/j.rser.2015.12.136

Andersson, J., Akesson, J., Diehl, M., 2012. Dynamic optimization with CasADi. 
Proc. IEEE Conf. Decis. Control 681-686. https://doi.org/10.1109/CDC.2012.6426534

Andersson, J.A.E., Gillis, J., Horn, G., Rawlings, J.B., Diehl, M., 2018. CasADi:A software framework for nonlinear optimization and optimal control. Math. Program. Comput.

Asadi, M., 2005. Beet-Sugar Handbook, Beet-Sugar Handbook. John Wiley \& Sons. https://doi.org/10.1002/9780471790990

Ashok, S., Banerjee, R., 2003. Optimal operation of industrial cogeneration for load management. IEEE Trans. Power Syst. 18, 931-937. https://doi.org/10.1109/TPWRS.2003.811169

Ashok, S., Banerjee, R., 2001. An optimization mode for industrial load management. IEEE Trans. Power Syst. 16, 879-884. https://doi.org/10.1109/59.962440

Baldea, M., Harjunkoski, I., 2014. Integrated production scheduling and process control: A systematic review. Comput. Chem. Eng. 71, 377-390. https://doi.org/10.1016/j.compchemeng.2014.09.002

Basu, M., 2016. Group search optimization for combined heat and power economic dispatch. Int. J. Electr. Power Energy Syst. 78, 138-147. https://doi.org/10.1016/j.ijepes.2015.11.069

Beal, L., Hill, D., Martin, R., Hedengren, J., 2018. GEKKO Optimization Suite. Processes 6, 106. https://doi.org/10.3390/pr6080106

Biegler, L.T., 2010. Nonlinear Programming: concepts, algorithms, and applications to chemical processes. Siam.

Bindlish, R., 2016. Power scheduling and real-time optimization of industrial cogeneration plants. Comput. Chem. Eng. 87, 257-266. https://doi.org/10.1016/j.compchemeng.2015.12.023

Brée, L.C., Perrey, K., Bulan, A., Mitsos, A., 2019. Demand side management and operational mode switching in chlorine production. AIChE J. 65, 1-14. https://doi.org/10.1002/aic.16352

Bruno, J.C., Fernandez, F., Castells, F., Grossmann, I.E., 1998. A rigorous minlp model for the optimal synthesis and operation of utility plants. Chem. Eng. Res. Des. 76, 246-258. https://doi.org/10.1205/026387698524901

Caspari, A., Offermanns, C., Schäfer, P., Mhamdi, A., Mitsos, A., 2019. A flexible air separation process: 2. Optimal operation using economic model predictive control. AIChE J. 65, 1-14. https://doi.org/10.1002/aic.16721

Caspari, A., Tsay, C., Mhamdi, A., Baldea, M., Mitsos, A., 2020. The integration of scheduling and control: Top-down vs. bottom-up. J. Process Control 91, 5062. https://doi.org/10.1016/j.jprocont.2020.05.008 
Castro, N., Villadiego, L., 2013. Amarga Dulzura: Una historia sobre el origen del azúcar.

Castro, P.M., Harjunkoski, I., Grossmann, I.E., 2011. Optimal scheduling of continuous plants with energy constraints. Comput. Chem. Eng. 35, 372387. https://doi.org/10.1016/j.compchemeng.2010.05.008

Cellier, F.E., 1991. Continuous System Modeling, Continuous System Modeling. Springer New York. https://doi.org/10.1007/978-1-4757-3922-0

Cervantes, A., Biegler, L.T., 2000. Optimization Strategies for Dynamic Systems. SpringerReference 1 , $1-22$. https://doi.org/10.1007/springerreference_72566

Chaibakhsh, A., Ghaffari, A., 2008. Steam turbine model. Simul. Model. Pract. Theory 16, 1145-1162. https://doi.org/10.1016/j.simpat.2008.05.017

Christidis, A., Koch, C., Pottel, L., Tsatsaronis, G., 2012. The contribution of heat storage to the profitable operation of combined heat and power plants in liberalized electricity markets. Energy 41, 75-82. https://doi.org/10.1016/j.energy.2011.06.048

Conejo, A.J., Baringo, L., 2018. Power System Operations, Power Electronics and Power Systems. Springer International Publishing, Cham. https://doi.org/10.1007/978-3-319-69407-8

Cozad, A., Sahinidis, N. V., Miller, D.C., 2015. A combined first-principles and data-driven approach to model building. Comput. Chem. Eng. 73, 116-127. https://doi.org/10.1016/j.compchemeng.2014.11.010

Cozad, A., Sahinidis, N. V, 2018. A global MINLP approach to symbolic regression. Math. Program. 170, 97-119. https://doi.org/10.1007/s10107-018-1289-x

de Prada, C., Hose, D., Gutierrez, G., Pitarch, J.L., 2018. Developing Grey-Box Dynamic Process Models. IFAC-PapersOnLine 51, 523-528. https://doi.org/10.1016/j.ifacol.2018.03.088

de Prada, C., Mazaeda, R., Cristea, S.P., 2019. Receding horizon scheduling of processes with shared resources. Comput. Chem. Eng. 125, 1-12. https://doi.org/10.1016/j.compchemeng.2019.03.005

der Poel, P.W., 1998. Sugar technology. Beet and cane sugar manufacture/PW van der Poel, H. Schiweck, T. Schwartz. Berlin Verlag Dr. Albert Vartens KG.

Diaz C., J.L., Ocampo-Martinez, C., Panten, N., Weber, T., Abele, E., 2019. Optimal operation of combined heat and power systems: An optimization-based control strategy. Energy Convers. Manag. 199, 111957. https://doi.org/10.1016/j.enconman.2019.111957

Ding, Y.M., Hong, S.H., Li, X.H., 2014. A demand response energy management scheme for industrial facilities in smart grid. IEEE Trans. Ind. Informatics 10, 
2257-2269. https://doi.org/10.1109/TII.2014.2330995

Dorfman, R., Samuelson, P., Solow, R., 1958. Linear programming and economic analysis. New York: McGraw-Hill.

Du, J., Park, J., Harjunkoski, I., Baldea, M., 2015. A time scale-bridging approach for integrating production scheduling and process control. Comput. Chem. Eng. 79, 59-69. https://doi.org/10.1016/j.compchemeng.2015.04.026

Dvořák, M., Havel, P., 2012. Combined heat and power production planning under liberalized market conditions. Appl. Therm. Eng. 43, 163-173. https://doi.org/10.1016/j.applthermaleng.2011.12.016

Ellis, M., Durand, H., Christofides, P.D., 2014. A tutorial review of economic model predictive control methods. J. Process Control 24, 1156-1178. https://doi.org/10.1016/j.jprocont.2014.03.010

Empresarios Agrupados, 2020. EcosimPro | PROOSIS Modelling and Simulation Software [WWW Document]. URL https://www.ecosimpro.com/ (accessed 9.9.20).

Energía y Sociedad, 2014a. 6.2. Formación de precios en los mercados mayoristas a plazo de electricidad | Energía y Sociedad [WWW Document]. URL http://www.energiaysociedad.es/manenergia/6-2-formacion-de-preciosen-los-mercados-mayoristas-a-plazo-de-electricidad/ (accessed 3.2.20).

Energía y Sociedad, 2014b. 6.5. Mecanismos de ajuste de demanda y producción I Energía y Sociedad [WWW Document]. URL http://www.energiaysociedad.es/manenergia/6-5-mecanismos-de-ajustede-demanda-y-produccion/ (accessed 3.2.20).

Energía y Sociedad, 2014c. 6.1. Formación de precios en el mercado mayorista diario de electricidad | Energía y Sociedad [WWW Document]. URL http://www.energiaysociedad.es/manenergia/6-1-formacion-de-preciosen-el-mercado-mayorista-diario-de-electricidad/ (accessed 3.2.20).

Fang, X., Misra, S., Xue, G., Yang, D., 2012. Smart grid - The new and improved power grid: A survey. IEEE Commun. Surv. Tutorials 14, 944-980. https://doi.org/10.1109/SURV.2011.101911.00087

Faulwasser, T., Korda, M., Jones, C.N., Bonvin, D., 2017. On turnpike and dissipativity properties of continuous-time optimal control problems. Automatica 81 , 297-304. https://doi.org/10.1016/j.automatica.2017.03.012

Floudas, C.A., 1995. Nonlinear and mixed-integer optimization: fundamentals and applications. Oxford University Press.

Frangopoulos, C.A., 2012. A method to determine the power to heat ratio, the cogenerated electricity and the primary energy savings of cogeneration 
systems after the European Directive. Energy 45, 52-61. https://doi.org/10.1016/j.energy.2011.12.044

Frankenfeld, T., Voss, C., 2004. Electrical power consumption-an European benchmarking-exercise-Part I: Survey. Zuckerindustrie 129, 407-414.

Gallego, J., Victoria, M., 2012. Entiende el Mercado Eléctrico 51.

Gambini, M., Vellini, M., 2014. High efficiency cogeneration: Performance assessment of industrial cogeneration power plants. Energy Procedia 45, 1255-1264. https://doi.org/10.1016/j.egypro.2014.01.131

Ghobeity, A., Mitsos, A., 2010. Optimal time-dependent operation of seawater reverse osmosis. Desalination 263, 76-88. https://doi.org/10.1016/j.desal.2010.06.041

Godoy, E., Benz, S.J., Scenna, N.J., 2011. A strategy for the economic optimization of combined cycle gas turbine power plants by taking advantage of useful thermodynamic relationships. Appl. Therm. Eng. 31, 852-871. https://doi.org/10.1016/j.applthermaleng.2010.11.004

Gvozdenac, D., Gvozdenac Urosevic, B., Menke, C., Urosevic, D., Bangviwat, A., 2017. High efficiency cogeneration : CHP and non-CHP energy. Energy 135, 269-278.

Hadera, H., Ekström, J., Sand, G., Mäntysaari, J., Harjunkoski, I., Engell, S., 2019. Integration of production scheduling and energy-cost optimization using Mean Value Cross Decomposition. Comput. Chem. Eng. 129. https://doi.org/10.1016/j.compchemeng.2019.05.002

Hadera, H., Harjunkoski, I., Sand, G., Grossmann, I.E., Engell, S., 2015. Optimization of steel production scheduling with complex time-sensitive electricity cost. Comput. Chem. Eng. 76, 117-136. https://doi.org/10.1016/j.compchemeng.2015.02.004

Harjunkoski, I., Maravelias, C.T., Bongers, P., Castro, P.M., Engell, S., Grossmann, I.E., Hooker, J., Méndez, C., Sand, G., Wassick, J., 2014. Scope for industrial applications of production scheduling models and solution methods. Comput. Chem. Eng. 62, 161-193. https://doi.org/10.1016/j.compchemeng.2013.12.001

Hart, W.E., Laird, C.D., Watson, J.-P., Woodruff, D.L., Hackebeil, G.A., Nicholson, B.L., Siirola, J.D., 2017. Pyomo - Optimization Modeling in Python 67. https://doi.org/10.1007/978-3-319-58821-6

Hart, W.E., Watson, J.P., Woodruff, D.L., 2011. Pyomo: Modeling and solving mathematical programs in Python. Math. Program. Comput. 3, 219-260. https://doi.org/10.1007/s12532-011-0026-8

Hernández, S., Pablos, C., Acebes, L.F., 2019. Interfaz para un optimizador que 
gestiona la producción de electricidad en una fábrica azucarera, in: XL Jornadas de Automática: Libro de Actas, Ferrol, 4-6 de Septiembre de 2019. pp. 506-512.

Isermann, R., Münchhof, M., 2010. Identification of dynamic systems: an introduction with applications. Springer Science \& Business Media.

Jena, C., Basu, M., Panigrahi, C.K., 2016. Differential evolution with Gaussian mutation for combined heat and power economic dispatch. Soft Comput. 20, 681-688. https://doi.org/10.1007/s00500-014-1531-2

Kehlhofer, R., Bachmann, R., Nielsen, H., Warner, J., 2009. Combined-Cycle Gas \& Steam Turbine Power Plants.

Kelley, M.T., Pattison, R.C., Baldick, R., Baldea, M., 2018. An MILP framework for optimizing demand response operation of air separation units. Appl. Energy 222, 951-966. https://doi.org/10.1016/j.apenergy.2017.12.127

Kia, M., Nazar, M.S., Sepasian, M.S., Heidari, A., Siano, P., 2017. Optimal day ahead scheduling of combined heat and power units with electrical and thermal storage considering security constraint of power system. Energy 120, 241-252. https://doi.org/10.1016/j.energy.2016.11.079

Kim, J.S., Edgar, T.F., 2014. Optimal scheduling of combined heat and power plants using mixed-integer nonlinear programming. Energy 77, 675-690. https://doi.org/10.1016/j.energy.2014.09.062

Leenders, L., Bahl, B., Hennen, M., Bardow, A., 2019. Coordinating scheduling of production and utility system using a Stackelberg game. Energy 175, 12831295. https://doi.org/10.1016/j.energy.2019.03.132

Leibman, M.J., Edgar, T.F., Lasdon, L.S., 1992. Efficient data reconciliation and estimation for dynamic processes using nonlinear programming techniques. Comput. Chem. Eng. 16, 963-986. https://doi.org/10.1016/0098-1354(92)80030-D

Leo, E., Engell, S., 2018. Integrated day-ahead energy procurement and production scheduling. At-Automatisierungstechnik 66, 950-963. https://doi.org/10.1515/auto-2018-0016

Ljung, L., 1999. System identification: theory for the user (second edition). Automatica. https://doi.org/10.1016/S0005-1098(01)00214-X

Ljung, L., 1988. System identification toolbox. Matlab user's Guid.

Lobato Miguélez, E., Egido Cortés, I., Rouco Rodríguez, L., López Camino, G., 2008. An overview of ancillary services in Spain. Electr. Power Syst. Res. 78, 515-523. https://doi.org/10.1016/j.epsr.2007.03.009

Lora, E.E.S., Rocha, M.H., Palacio, J.C.E., Venturini, O.J., Renó, M.L.G., del Olmo, O.A., 2013. The sugar and alcohol industry in the biofuels and cogeneration 
era: a paradigm change (part I). Sugar Ind. 139, 28-36. https://doi.org/10.36961/si15193

Lucia, S., 2015. Robust Multi-stage Nonlinear Model Predictive Control.

MacGregor, P.R., Puttgen, H.B., 1991. A Spot Price Based Control Mechanism for Electric Utility Systems with Small Power Producing Facilities. IEEE Power Eng. Rev. 11, 66. https://doi.org/10.1109/MPER.1991.90626

Majidi, M., Mohammadi-Ivatloo, B., Anvari-Moghaddam, A., 2019. Optimal robust operation of combined heat and power systems with demand response programs. Appl. Therm. Eng. 149, 1359-1369. https://doi.org/10.1016/j.applthermaleng.2018.12.088

Marshman, D.J., Chmelyk, T., Sidhu, M.S., Gopaluni, R.B., Dumont, G.A., 2010. Energy optimization in a pulp and paper mill cogeneration facility. Appl. Energy 87, 3514-3525. https://doi.org/10.1016/j.apenergy.2010.04.023

Mazaeda, R., 2010. Library of Models of the Sugar House of the Sugar Industry for Operator's Training.

Merino, A., 2008. Librería de Modelos del Cuarto de Remolacha de una Industria Azucarera para un Simulador de Entrenamiento de Operarios 1-14.

Merino, A., Acebes, L.F., 2003. Dynamic Simulation of an RT extractor. Zuckerindustrie 443-452.

Merkert, L., Harjunkoski, I., Isaksson, A., S??ynevirta, S., Saarela, A., Sand, G., Säynevirta, S., Saarela, A., Sand, G., 2015. Scheduling and energy - Industrial challenges and opportunities. Comput. Chem. Eng. 72, 183-198. https://doi.org/10.1016/j.compchemeng.2014.05.024

Meyabadi, A.F., Deihimi, M.H., 2017. A review of demand-side management: Reconsidering theoretical framework. Renew. Sustain. Energy Rev. 80, 367379. https://doi.org/10.1016/j.rser.2017.05.207

Mitra, S., Grossmann, I.E., Pinto, J.M., Arora, N., 2012. Optimal production planning under time-sensitive electricity prices for continuous powerintensive processes. Comput. Chem. Eng. 38, 171-184. https://doi.org/10.1016/j.compchemeng.2011.09.019

Mitra, S., Sun, L., Grossmann, I.E., 2013. Optimal scheduling of industrial combined heat and power plants under time-sensitive electricity prices. Energy 54, 194-211. https://doi.org/10.1016/j.energy.2013.02.030

Moita, R.D., Matos, H.A., Fernandes, C., Nunes, C.P., Prior, J.M., 2005. Dynamic modelling and simulation of a cogeneration system integrated with a salt recrystallization process. Comput. Chem. Eng. 29, 1491-1505. https://doi.org/10.1016/j.compchemeng.2005.02.015

Navia López, D.A., 2013. Handling uncertainties in process optimization 1-304. 
Nelles, O., 2013. Nonlinear system identification: from classical approaches to neural networks and fuzzy models. Springer Science \& Business Media.

NEMO Commitee, 2019. Euphemia Public Description.

Official Journal of the European Union, 2012. Directive 2012/27/EU of the European Parliament and of the Council of 25 October 2012 on energy efficiency. Off. J. Eur. Union 1-56.

OMIE, 2020. Electricity market | OMIE [WWW Document]. URL https://www.omie.es/en/mercado-de-electricidad (accessed 2.24.21).

OPC Foundation, 2014. OPC Foundation [WWW Document]. URL https://opcfoundation.org/about/opc-technologies/opc-ua/ (accessed 3.13.21).

Pablos, C., Felipe Acebes, L., Merino, A., Acebes, L.F., Merino, A., Felipe Acebes, L., Merino, A., 2017. Sugar plant simulator for energy management purposes. 29th Eur. Model. Simul. Symp. 370-379.

Pablos, C., Merino, A., Acebes, L.F., 2019. Modeling On-Site Combined Heat and Power Systems Coupled to Main Process Operation. Processes 7, 218. https://doi.org/10.3390/pr7040218

Pablos, C., Merino, A., Acebes, L.F., Pitarch, L., Biegler, L.T., 2021. Dynamic Optimization Approach to Coordinate Industrial Production and Cogeneration Operation Under Electricity Price Fluctuations. Comput. Chem. Eng. 107292. https://doi.org/10.1016/j.compchemeng.2021.107292

Pan, R., Li, Z., Cao, J., Zhang, H., Xia, X., 2019. Electrical load tracking scheduling of steel plants under time-of-use tariffs. Comput. Ind. Eng. 137, 106049. https://doi.org/10.1016/j.cie.2019.106049

Pattison, R.C., Touretzky, C.R., 2016. Moving Horizon Closed-Loop Production Scheduling Using Dynamic Process Models. AIChE J. 63, 639-651. https://doi.org/10.1002/aic.15408

Pattison, R.C., Touretzky, C.R., Johansson, T., Harjunkoski, I., Baldea, M., 2016. Optimal Process Operations in Fast-Changing Electricity Markets: Framework for Scheduling with Low-Order Dynamic Models and an Air Separation Application. Ind. Eng. Chem. Res. 55, 4562-4584. https://doi.org/10.1021/acs.iecr.5b03499

Pelayo, S., 1999. Modelado y Simulación Dinámica de una Caldera de Vapor Industrial. Universidad de Valladolid.

Perry, R.H., Green, D.W., Maloney, J.O., 2015. Perry's chemical engineers' handbook. McGraw-Hill New York.

Piperagkas, G.S., Anastasiadis, A.G., Hatziargyriou, N.D., 2011. Stochastic PSO- 
based heat and power dispatch under environmental constraints incorporating CHP and wind power units. Electr. Power Syst. Res. 81, 209218. https://doi.org/10.1016/j.epsr.2010.08.009

Pitarch, J.L., Sala, A., de Prada, C., 2019. A systematic grey-box modeling methodology via data reconciliation and SOS constrained regression. Processes 7, 1-23. https://doi.org/10.3390/PR7030170

Pontryagin, V.V., Boltyanskii, Gamkrelidze, R., Mishchenko, E., 1962. The mathematical theory of optimal processes, Interscien. ed. New York.

Rawlings, J.B., Amrit, R., 2009. Optimizing Process Economic Performance Using Model Predictive Control, in: Magni, L., Raimondo, D.M., Allgower, F. (Eds.), Nonlinear Model Predictive Control. pp. 120-138. https://doi.org/https://doi.org/10.1007/978-3-642-01094-1_10

REE, 2019. Red Eléctrica at a glance [WWW Document]. URL https://www.ree.es/en/about-us/ree-2-minutes (accessed 2.25.20).

Rodríguez-Blanco, T., 2017. Modifier Adaptation for process optimization with uncertainty.

Rong, A., Lahdelma, R., 2007. An efficient envelope-based Branch and Bound algorithm for non-convex combined heat and power production planning. Eur. J. Oper. Res. 183, 412-431. https://doi.org/10.1016/j.ejor.2006.09.072

Sadeghian, H.R., Ardehali, M.M., 2016. A novel approach for optimal economic dispatch scheduling of integrated combined heat and power systems for maximum economic profit and minimum environmental emissions based on Benders decomposition. Energy 102, 10-23. https://doi.org/10.1016/j.energy.2016.02.044

Salgado, F., Pedrero, P., 2008. Short-term operation planning on cogeneration systems: A survey. Electr. Power Syst. Res. 78, 835-848. https://doi.org/10.1016/j.epsr.2007.06.001

Sánchez, H., 2019. Interfaz gráfica de aplicaciones de control desarrolladas con Python. University of Valladolid.

Santos, M.I., Uturbey, W., 2018. A practical model for energy dispatch in cogeneration plants. Energy 151, 144-159. https://doi.org/10.1016/j.energy.2018.03.057

Sashirekha, A., Pasupuleti, J., Moin, N.H., Tan, C.S., 2013. Combined heat and power (CHP) economic dispatch solved using Lagrangian relaxation with surrogate subgradient multiplier updates. Int. J. Electr. Power Energy Syst. 44, 421-430. https://doi.org/10.1016/j.ijepes.2012.07.038

Schäfer, P., Caspari, A., Mhamdi, A., Mitsos, A., 2019. Economic nonlinear model predictive control using hybrid mechanistic data-driven models for optimal 
operation in real-time electricity markets: In-silico application to air separation processes. J. Process Control 84, 171-181. https://doi.org/10.1016/j.jprocont.2019.10.008

Shaabani, Y. ali, Seifi, A.R., Kouhanjani, M.J., 2017. Stochastic multi-objective optimization of combined heat and power economic/emission dispatch. Energy 141, 1892-1904. https://doi.org/10.1016/j.energy.2017.11.124

Siano, P., 2014. Demand response and smart grids - A survey. Renew. Sustain. Energy Rev. 30, 461-478. https://doi.org/10.1016/j.rser.2013.10.022

Subbaraj, P., Rengaraj, R., Salivahanan, S., 2009. Enhancement of combined heat and power economic dispatch using self adaptive real-coded genetic algorithm. Appl. Energy 86, 915-921. https://doi.org/10.1016/j.apenergy.2008.10.002

Sun, Z., Li, L., Bego, A., Dababneh, F., 2015. Customer-side electricity load management for sustainable manufacturing systems utilizing combined heat and power generation system. Int. J. Prod. Econ. 165, 112-119. https://doi.org/10.1016/j.ijpe.2015.04.002

Tan, K.C., Li, Y., 2002. Grey-box model identification via evolutionary computing. Control Eng. Pract. 10, 673-684. https://doi.org/10.1016/S09670661(02)00031-X

Thomas, P.J., 1999. Simulation of industrial processes for control engineers. Elsevier.

Tina, G.M., Passarello, G., 2012. Short-term scheduling of industrial cogeneration systems for annual revenue maximisation. Energy 42, 46-56. https://doi.org/10.1016/j.energy.2011.10.025

Tolsma, J.E., Barton, P.I., 1998. On computational differentiation. Comput. Chem. Eng. 22, 475-490. https://doi.org/10.1016/S0098-1354(97)00264-0

Tsay, C., Kumar, A., Flores-Cerrillo, J., Baldea, M., 2019. Optimal demand response scheduling of an industrial air separation unit using data-driven dynamic models. Comput. Chem. Eng. 126, 22-34. https://doi.org/10.1016/j.compchemeng.2019.03.022

Urbaniec, K., 1989. Modern energy economy in beet sugar factories.

Venkatesh, B.N., Chankong, V., 1995. Decision models for management of cogeneration plants. IEEE Trans. Power Syst. 10, 1250-1256. https://doi.org/10.1109/59.466530

Wang, Z., Gao, F., Zhai, Q., Guan, X., Wu, J., Liu, K., 2013. Electrical load tracking analysis for demand response in energy intensive enterprise. IEEE Trans. Smart Grid 4, 1917-1927. https://doi.org/10.1109/TSG.2013.2257186

Wills, A., Schön, T.B., Ljung, L., Ninness, B., 2013. Identification of Hammerstein- 
Wiener models. Automatica 49, 70-81. https://doi.org/10.1016/j.automatica.2012.09.018

Wonderware, 2017. Wonderware Intouch System Platform [WWW Document]. URL platform/novedades/ https://www.wonderware.es/hmi-scada/system-

Yusta, J.M., De Oliveira-De Jesus, P.M., Khodr, H.M., 2008. Optimal energy exchange of an industrial cogeneration in a day-ahead electricity market. Electr. Power Syst. Res. 78, 1764-1772.

Yusta, J.M., Torres, F., Khodr, H.M., 2010. Optimal methodology for a machining process scheduling in spot electricity markets. Energy Convers. Manag. 51, 2647-2654. https://doi.org/10.1016/j.enconman.2010.05.030

Zamarreño, J.M., Pablos, C., Merino, A., Acebes, L.F., de Prada, C., 2017. INFRAESTRUCTURA PARA EXPLOTACIÓN DE DATOS DE UN SIMULADOR AZUCARERO.

Zhang, B.J., Hua, B., 2007. Effective MILP model for oil refinery-wide production planning and better energy utilization. J. Clean. Prod. 15, 439-448. https://doi.org/10.1016/j.jclepro.2005.08.004

Zhang, B.J., Luo, X.L., Chen, X.Z., Chen, Q.L., 2013. Coupling process plants and utility systems for site scale steam integration. Ind. Eng. Chem. Res. 52, 14627-14636. https://doi.org/10.1021/ie401952h

Zhang, Q., Grossmann, I.E., Zhang Q., G.I.E., Zhang, Q., Grossmann, I.E., 2016. Planning and scheduling for industrial demand side management: Advances and challenges. Altern. Energy Sources Technol. Process Des. Oper. 383414. https://doi.org/10.1007/978-3-319-28752-2_14

Zhao, S., Grossmann, I.E., Tang, L., 2018. Integrated scheduling of rolling sector in steel production with consideration of energy consumption under timeof-use electricity prices. Comput. Chem. Eng. 111, 55-65. https://doi.org/10.1016/j.compchemeng.2017.12.018

Zhu, Y., Legg, S., Laird, C.D., 2010. A Multiperiod Nonlinear Programming Approach for Operation of Air Separation Plants with Variable Power Pricing. AIChE J. 57, 2421-2430. https://doi.org/10.1002/aic.12464

Zhuge, J., lerapetritou, M.G., 2012. Integration of scheduling and control with closed loop implementation. Ind. Eng. Chem. Res. 51, 8550-8565. https://doi.org/10.1021/ie3002364

Zulkafli, N.I., Hanak, D.P., Kopanos, G.M., 2020. Efficient decomposition strategy for scheduling of multistage production system and combined heat and power. Comput. Chem. Eng. 133, 106634. https://doi.org/10.1016/j.compchemeng.2019.106634 
Zulkafli, N.I., Kopanos, G.M., 2017. Integrated condition-based planning of production and utility systems under uncertainty. J. Clean. Prod. 167, 776805. https://doi.org/10.1016/j.jclepro.2017.08.152

Zulkafli, N.I., Kopanos, G.M., 2016. Planning of production and utility systems under unit performance degradation and alternative resource-constrained cleaning policies. Appl. Energy 183, 577-602. https://doi.org/10.1016/j.apenergy.2016.08.060 


\section{Appendix A}

\section{Variables and Parameters}

Here, we list the variables and parameters needed for the application of the methodology developed on the simulated case study.

Table A-1. Variables notation.

\begin{tabular}{lll}
\hline Variable & Description & Units \\
\hline$\Delta$ Var & $\begin{array}{l}\text { Increase in the value of the variable Var w.r.t the } \\
\text { equilibrium point (identification models) }\end{array}$ & \\
$e$ & Split-range controller error & barA \\
$E_{C}$ & Electricity commitment & $\mathrm{kW}$ \\
$E_{C H P}$ & Power generated in cogeneration mode & $\mathrm{kW}$ \\
$E_{p}$ & Electricity consumption of the sugar factory & $\mathrm{kW}$ \\
$E_{S}$ & Electricity surplus & $\mathrm{kW}$ \\
$E_{T u}$ & Power generated in turbines & $\mathrm{kW}$ \\
$m_{S t}$ & Accumulated mass beet in the storage zone & $\mathrm{T}$ \\
$F_{C H P}$ & Energy obtained from fuel in cogeneration mode & $\mathrm{kJ} / \mathrm{s}$ \\
$F_{\text {plant }}$ & Energy obtained from fuel & $\mathrm{kJ} / \mathrm{s}$
\end{tabular}




\begin{tabular}{|c|c|c|}
\hline$H_{S B O}$ & Enthalpy of the steam leaving the boilers & $\mathrm{kJ} / \mathrm{kg}$ \\
\hline$H_{S B y}$ & Enthalpy of the steam at bypass valve & $\mathrm{kJ} / \mathrm{kg}$ \\
\hline$H_{S R e}$ & Enthalpy of the steam at relief valve & $\mathrm{kJ} / \mathrm{kg}$ \\
\hline$H_{\text {SSaIn }}$ & Steam enthalpy at saturator input & $\mathrm{kJ} / \mathrm{kg}$ \\
\hline$H_{\text {SSaOut }}$ & Steam enthalpy at saturator output & $\mathrm{kJ} / \mathrm{kg}$ \\
\hline$H_{\text {STUIn }}$ & Enthalpy of the steam that enters in the turbines. & $\mathrm{kJ} / \mathrm{kg}$ \\
\hline$H_{\text {STUOut }}$ & Enthalpy of the steam that leaves the turbines. & $\mathrm{kJ} / \mathrm{kg}$ \\
\hline$H_{W B o}$ & Boiler water enthalpy & $\mathrm{kJ} / \mathrm{kg}$ \\
\hline$m_{S t}$ & Beet mass accumulated in the storage area & $\mathrm{kg}$ \\
\hline$\mu E_{C H P}$ & Power efficiency of the CHP & $\%$ \\
\hline$\mu_{G}$ & Global efficiency & $\%$ \\
\hline$\mu Q_{C H P}$ & Heat efficiency of the CHP & $\%$ \\
\hline$O p_{B y}$ & Opening of the bypass valve & $\%$ \\
\hline$O p_{R e}$ & Opening of the relief valve & $\%$ \\
\hline$P_{I V}$ & Pressure inside the $4^{\text {th }}$ evaporation effect & barA \\
\hline PES & Primary Saving Energy index & \\
\hline$P_{\text {SSaOut }}$ & Pressure of the steam leaving the saturator & barA \\
\hline$P_{\text {SSaOutRef }}$ & Split-range controller reference & barA \\
\hline$P_{\text {STUIn }}$ & Steam pressure at turbines input & barA \\
\hline$P_{\text {STuout }}$ & Steam pressure at turbines output & barA \\
\hline$T_{S B O}$ & Temperature of the steam leaving the boilers & ${ }^{\circ} \mathrm{C}$ \\
\hline$T_{\text {STuIn }}$ & Steam temperature at turbines input & ${ }^{\circ} \mathrm{C}$ \\
\hline$T_{\text {STuOut }}$ & Steam temperature at turbines output & \\
\hline$Q_{C H P}$ & Heat generated in cogeneration mode & kW \\
\hline$Q_{p}$ & Sugar factory heat energy consumption & $\mathrm{kW}$ \\
\hline$Q_{S B o}$ & Heat of the steam current leaving the boilers & kW \\
\hline$S_{0}, \ldots, S_{5}$ & Auxiliary variables for complementarity constraints & \\
\hline StoPenUp & $\begin{array}{l}\text { Measures the residence time excess w.r.t the } \\
\text { maximum value }\end{array}$ & \\
\hline
\end{tabular}




\begin{tabular}{|c|c|c|}
\hline StoPenLo & $\begin{array}{l}\text { Measures the residence time defect w.r.t the } \\
\text { minimum value }\end{array}$ & \\
\hline$\tau_{S t}$ & Storage residence time & $\mathrm{h}$ \\
\hline$u_{V a r}$ & Input vector w.r.t variable Var (Identification models) & \\
\hline$v$ & Split-range controller output signal & $\%$ \\
\hline$v_{i}$ & Split-range controller integral action & $\%$ \\
\hline$W_{B S t I n}$ & Beet mass flow entering in the storage zone & $\mathrm{kg} / \mathrm{s}$ \\
\hline$W_{\text {BStout }}$ & Beet mass flow leaving the storage zone & $\mathrm{kg} / \mathrm{s}$ \\
\hline$W_{G}$ & Natural gas mass flow entering in boilers & $\mathrm{kg} / \mathrm{s}$ \\
\hline$W_{S B o}$ & Steam mass flow leaving the boilers & $\mathrm{kg} / \mathrm{s}$ \\
\hline$W_{S B y}$ & Steam mass flow at bypass valve & $\mathrm{kg} / \mathrm{s}$ \\
\hline$W_{S R e}$ & Steam mass flow at relief valve & $\mathrm{kg} / \mathrm{s}$ \\
\hline$W_{\text {SSaIn }}$ & Steam mass flow at saturator input & $\mathrm{kg} / \mathrm{s}$ \\
\hline$W_{\text {SSaOut }}$ & Steam mass flow at saturator output & $\mathrm{kg} / \mathrm{s}$ \\
\hline$W_{\text {STuIn }}$ & Steam mass flow entering in the turbines & $\mathrm{kg} / \mathrm{s}$ \\
\hline$W_{\text {STuout }}$ & Steam mass flow leaving the turbines & $\mathrm{kg} / \mathrm{s}$ \\
\hline$W_{W S a}$ & Water mass flow entering in the saturator & $\mathrm{kg} / \mathrm{s}$ \\
\hline$x_{\text {Var }}$ & $\begin{array}{l}\text { Internal state vector of Var variable (identification } \\
\text { models) }\end{array}$ & \\
\hline$y$ & Binary variable used for complementarity & \\
\hline
\end{tabular}


Table A- 2. Parameter values and notation.

\begin{tabular}{|c|c|c|c|}
\hline Name & Description & Value & Units \\
\hline$d_{G}$ & $\begin{array}{l}\text { Natural Gas density for the input } \\
\text { conditions }\end{array}$ & 3.65 & $\mathrm{~kg} / \mathrm{m}^{3}$ \\
\hline$E_{P_{e q}}$ & $E_{P}$ value at the identification point & $7,769.26$ & kW \\
\hline$H_{W B o}$ & $\begin{array}{l}\text { Specific enthalpy of the water used in } \\
\text { the boilers }\end{array}$ & 550.52 & $\mathrm{~kJ} / \mathrm{kg}$ \\
\hline$H_{W S a}$ & Water enthalpy at saturator input & 125.8 & $\mathrm{~kJ} / \mathrm{kg}$ \\
\hline$k p$ & $\begin{array}{l}\text { Split-range controller proportional } \\
\text { gain }\end{array}$ & 140 & $\% /$ bar \\
\hline$k$ & Polytropic index & 1.20 & \\
\hline$K v_{B y}$ & Bypass rated valve coefficient & 0.50 & $\mathrm{~kg} /(\mathrm{s} \cdot \mathrm{bar})$ \\
\hline$K v_{R e}$ & Relief rated valve coefficient & 5.00 & $\mathrm{~kg} /(\mathrm{s} \cdot \mathrm{bar})$ \\
\hline$K_{T u}$ & Turbines experimental parameter & 23.35 & $\left(\mathrm{~kg} / \mathrm{s} \cdot{ }^{\circ} \mathrm{C}\right) / \mathrm{bar}$ \\
\hline$\mu E_{R e f}$ & $\begin{array}{l}\text { Reference cogeneration power } \\
\text { efficiency }\end{array}$ & 0.53 & \\
\hline$\mu Q_{R e f}$ & $\begin{array}{l}\text { Reference cogeneration heat } \\
\text { efficiency }\end{array}$ & 0.87 & \\
\hline$\mu_{T u}$ & Efficiency of the steam turbine & 0.95 & \\
\hline$L H V_{G}$ & Natural Gas Lower Heating Value & $47,100.00$ & $\mathrm{~kJ} / \mathrm{kg}$ \\
\hline$H H V_{G}$ & Natural Gas Higher Heating Value & $52,200.00$ & $\mathrm{~kJ} / \mathrm{kg}$ \\
\hline$P_{I V}$ eq & $P_{I V}$ value at the identification point & 1.28 & barA \\
\hline$P_{a t m}$ & Atmospheric pressure & 1.00 & barA \\
\hline$P_{S B o}$ & Boilers operating pressure & 37.30 & barA \\
\hline$P r_{G}$ & Natural Gas Price & $\mathrm{N} / \mathrm{A}$ & $€ / \mathrm{kWh}$ \\
\hline$P r_{E}$ & Electricity Price & $\mathrm{N} / \mathrm{A}$ & $€ / \mathrm{kWh}$ \\
\hline
\end{tabular}




\begin{tabular}{|c|c|c|c|}
\hline$Q_{P_{e q}}$ & $Q_{p}$ value at the identification point & $67,779.55$ & $\mathrm{~kW}$ \\
\hline$\rho_{S R}$ & $\begin{array}{lcr}\text { Split } & \text { range } & \text { controller } \\
\text { complementarity weight } & \end{array}$ & 1 & \\
\hline$\rho_{S P}$ & $\begin{array}{l}\text { Storage penalty complementarity } \\
\text { weight }\end{array}$ & 250 & \\
\hline$\rho_{W_{\text {BStuOut }}}$ & Production rate moving weight & 1 & \\
\hline$\rho_{T_{S B o}}$ & $\begin{array}{l}\text { Steam boilers temperature moving } \\
\text { weight }\end{array}$ & 1 & \\
\hline$\rho_{P_{\text {SSaOut }}}$ & $\begin{array}{l}\text { Evaporation working pressure moving } \\
\text { weight }\end{array}$ & 1000 & \\
\hline$\rho_{E_{T u}}$ & $\begin{array}{l}\text { Power generated in turbines moving } \\
\text { weight }\end{array}$ & 0.01 & \\
\hline$\rho_{S t}$ & Storage penalty weight & 0.5 & \\
\hline$\rho_{T p}$ & Turnpike penalty weight & 0.2 & \\
\hline$T$ & Prediction horizon time & 86400 & $\mathrm{~s}$ \\
\hline$T i$ & Split-range controller integral gain & 10.00 & $\mathrm{~s}$ \\
\hline$\tau_{\min }$ & Slowest time dynamic of the plant & 18000 & $\mathrm{~s}$ \\
\hline$v_{\max }$ & $\begin{array}{l}\text { Maximum output signal of the split } \\
\text { range controller }\end{array}$ & 100.00 & $\%$ \\
\hline$W_{B S t I n}$ & Arrival of beet to the storage zone & $\mathrm{N} / \mathrm{A}$ & $\mathrm{T} / \mathrm{h}$ \\
\hline$W_{G_{e q}}$ & $W_{G}$ value at the identification point & 1.39 & $\mathrm{~kg} / \mathrm{s}$ \\
\hline
\end{tabular}





\section{Appendix B}

\section{Industrial roll-out concept applied to a beet-sugar process}

In chapter 5.1 and 6, we have explained the implementation of the dynamicintegrated approach in the simulated beet-sugar factory presented in chapter 4 . First, we have described the modeling process and, later, the optimization formulation and results. After knowing that the optimization module works in a simulated environment, it would be time to consider its roll-out in the real plant, topic introduced in Section 3.4. Unfortunately, in this thesis, we have only worked in silico, but, given the importance of the matter, we have considered convenient to reflect in this appendix about some important concepts related to the industrial roll-out, and present two additional tools that are not essential, but advisable to count with, to deploy the optimization tool developed. Furthermore, we explain the way that we consider these add-ons should communicate with each other in the industrial field, considering that, of course, in a real roll-out we would have to face additional problems. Therefore, the content of this appendix must not be seen as a guide or a methodology, but as a concept for the industrial roll-out of the optimization tool developed. 


\section{B.1 Architecture}

The architecture developed here is the application of the one presented in Section 3.4 to our simulated beet-sugar factory. Given that we do not count with a real process plant, we have limited the implementation to the development of the simulation and visualization modules, and its communication with the optimization tool. The real plant has been replaced by the simulation module. All the modules have been connected using the standard OPC Unified Architecture (OPC UA), which nowadays is widely implemented in the industry, and allows the exchange of great amount of data of different classes at high speed (OPC Foundation, 2014).

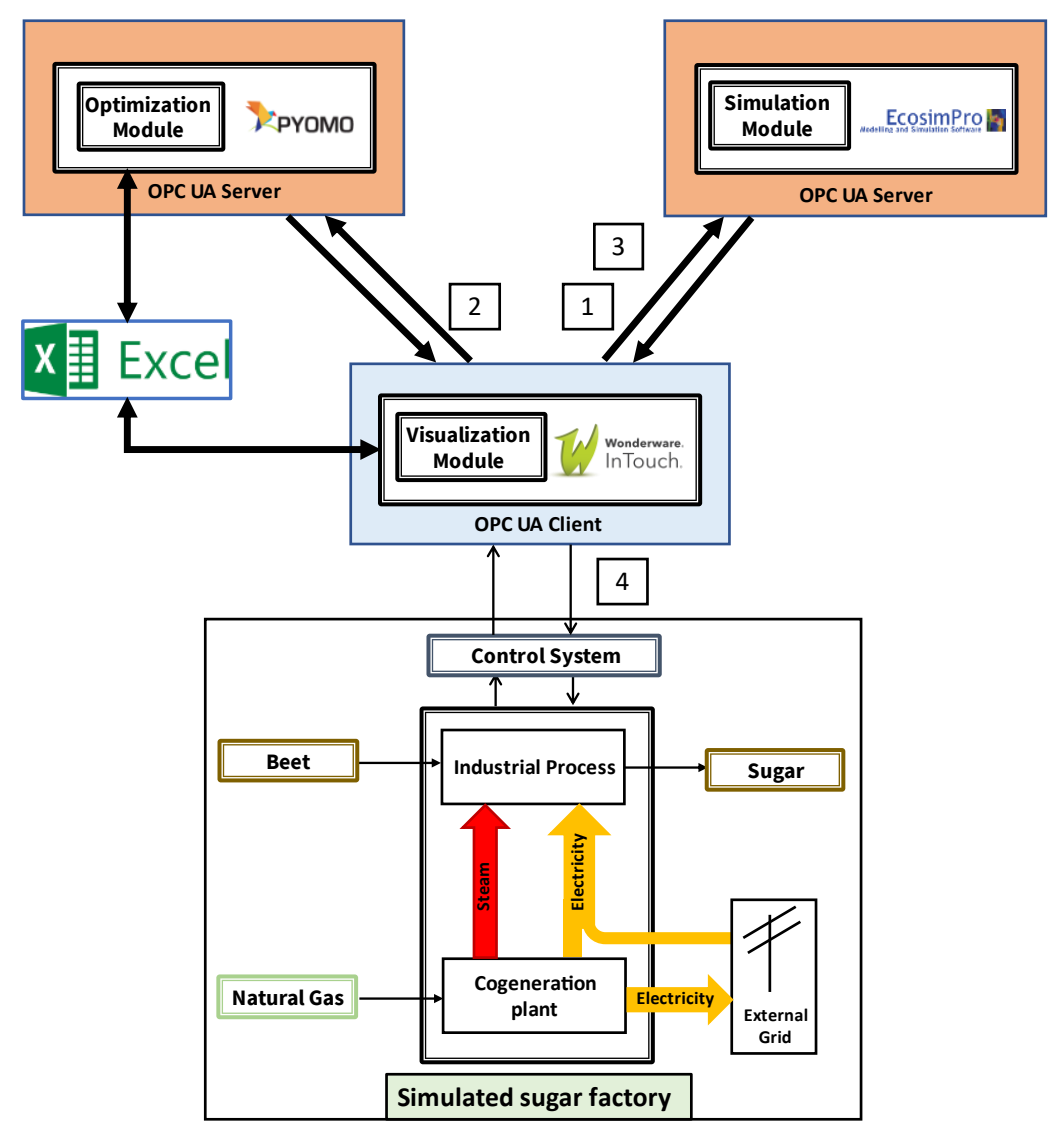

Figure B-1. Communication architecture between the software used. 
The simulation module has been implemented using EcosimPro ${ }^{\circledR}$, and it has been provided with an OPC UA server layer. It receives the current state of the process, an operation strategy, and the required simulation parameters from the visualization module, and returns the estimated response of the real process, which is used to predict the initial state for the optimizer or perform a what-if analysis. More information about the building process of the simulation module is given in the next section.

Regarding the optimization module, as mentioned in Section 6.3.4, it has been implemented using the Pyomo library under a Python environment. The optimization receives the estimated initial state, the expected beet input and electricity price, and some optimization parameters introduced by the operator from the visualization module and returns the optimal operation strategy. To communicate the optimization with the visualization and simulation module, a server layer has been added to this module using the Python library freeOPCUA.

With respect to the visualization module, it has been implemented using the Wonderware ${ }^{\circledR}$ Intouch $^{\circledR}$ System Platform (Wonderware, 2017), which in an industrial environment is used to manage infrastructures and real-time data from process plants. This software counts with a high-quality engine to create Human-Machine Interfaces attractive to the end-user. Specifically, the version used in this work is 2014 R2 SP1, which operates under the architecture ArchestrA, used as an interconnection platform between the internal modules. Such modules are used for the graphical interface design, to establish communication protocols, and for the management of historical data, among others.

With Intouch ${ }^{\circledR}$, several OPC UA clients can be created and communicated with different OPC UA servers simultaneously. In our case, we have created two different OPC UA clients, one to connect with the optimization module, and another one to connect with the simulation module. Such connections are managed by a gateway, where the different variables, methods, and objects exchanged are stocked. Furthermore, once stocked, the gateway uses internal protocols to establish the connection with the different Intouch ${ }^{\circledR}$ internal modules. However, due to the difficulties of Intouch ${ }^{\circledR}$ to deal with arrays and handle XY graphics, we have needed to use Microsoft ${ }^{\circledR}$ Excel $^{\circledR}$, which is natively compatible with Python and Intouch ${ }^{\circledR}$, to exchange such information with the optimizer and present some of the optimization results. 


\section{B.2 Simulation module}

The objective of this module is to have a dynamic and realistic model able to simulate the whole system behavior (Beet-sugar factory and CHP unit) depicted in Section 4.1 to estimate the initial conditions of the dynamic optimization problem, and test different operation strategies before being implemented (What-if analysis). Furthermore, in our particular case, along with the optimization process design, such simulator has been used to test the optimization module performance, and as the source of all data used in the model identification stage. Of course, with a real process the needed data would be collected from historical data instead.

As different strategies may be necessary to be tested in a relatively small amount of time, a simulator simple enough is necessary to carry out a lot of experiments without excessive computing time. However, an excessive model simplification could reduce the model accuracy in excess, so it is necessary to keep an equilibrium between accuracy and computation time. Thus, we decided that the mathematical model should be a first-principles dynamic one, but in case the model complexity exceeded the intended use, sufficiently validated empirical relations would be used. This implied that the formulation of the model should be based on ODEs, DAEs, algebraic equations, tables and events.

Given the magnitude of the system to be simulated, we decided to build the mathematical model in an incremental way, using models that could be instantiated from a library and composed in a hierarchical way. A further requirement for the simulation tool was the capacity to develop model libraries from scratch. Although using commercial libraries, which provide already validated model libraries, sometimes can be helpful, they frequently limit the characterization of the simulated process, and in certain cases, like the sugar industry, such libraries are very hard to find or non-existent. Another requirement was that the modeling tool should help in the task of symbolic manipulation of the global mathematical model to obtain the simulation model based on the input variables in an easier way.

These requirements implied to choose a modeling environment that implements an equation object-oriented modeling language (EOOBML), and a graphical interface that allowed the modeling based on the connection of components. The modeling tool should be linked to a simulation environment 
that facilitates the model execution, changes in parameter and input signals, and visualization of output signals. Also, it had to allow the recording of the results, so the data could be exploited with other tools like, for example, MATLAB ${ }^{\circledR}$. Furthermore, the simulation had to be able to be used as standalone from the modeling and simulation environment. In particular, it was desired to run the simulation with OPC UA connectivity, since OPC UA is supported by many industrial control systems and by optimization tools such as Pyomo. So, with all this in mind, the modeling and simulation environment selected was EcosimPro ${ }^{\circledR}$.

\section{Model description}

The simulator presented in this section is based on a previous project developed by the Sugar Technology Center and the University of Valladolid, where a complete sugar simulator was modeled to train control room operators of sugar factories (Acebes et al., 2011; Mazaeda, 2010; Merino, 2008). The problem with this simulator (training simulator from now on) is that because of the complexity of the models used, the simulation can hardly run faster than real time. Furthermore, it counts with features, like its capability to provoke failures in different parts of the plant, that increase the computational load and are not useful for this work purposes. Therefore, considering that such simulator was also modeled using EcosimPro ${ }^{\circledR}$, we decided to use part of the libraries already created and complement them with new models where necessary.

Thus, considering our modeling aims, and to simplify the model as much as possible, we decided to create a simulator mainly focused on the energy management (heat and electricity) of the beet-sugar process. The resulting mathematical model does not represent any concrete sugar factory, but a general one. A scheme of the process considered is presented in Figure 4-1. We used first-principles equations where more accuracy was needed (the evaporation stage and the cogeneration plant), and empirical expressions when it was only necessary to compute the heat and power demand (beet storage, diffusion, purification and crystallization stages). We used the EcosimPro ${ }^{\circledR}$ graphical interface to create a final model composed of many smaller ones, which is shown in Figure B-2. 


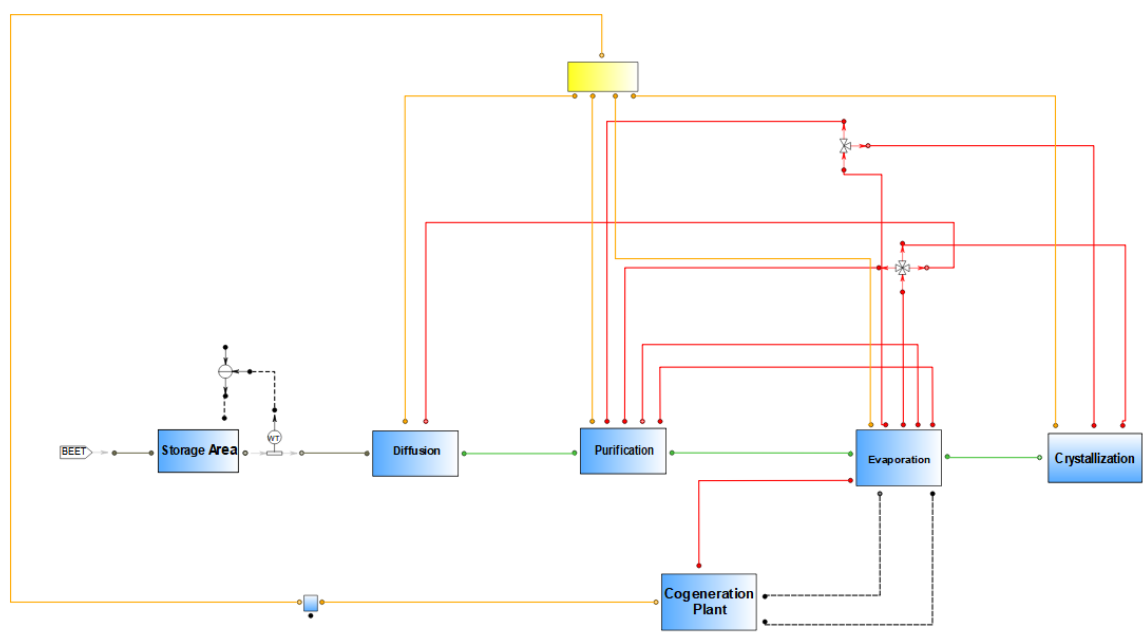

Figure B-2. Schematic model of the beet-sugar factory considered.

In Figure B-2, we can see that the different stages of the process have been encapsulated under smaller models with its own inputs and outputs. EcosimPro ${ }^{\circledR}$ exchanges the information of such input/output variables using Ports. In the schematic we can see different types of ports with different lines and colors depending on the variables exchanged. For example, the steam related variables have been communicated with steam ports (in red); the electricity related ones with electricity ports (in yellow); information about control signals with analog signal ports (in dashed-dotted black); and variables related to sugar juice with juice ports (in green). Furthermore, we can see the symbols of some modeled control items, like valves, transmitters, or controllers.

The objective of this section is to give some insights about the developed model without giving much detail. To do so, in the next sections we will cover the main aspects of each plant stage model. We recall that almost all the elemental models used to build the final model have been obtained from previous work that has already been validated. Therefore, for more details about the original models, the reader is referenced to such previous work.

\section{Beet reception and storage}

As mentioned in Section 4.1, the beet is transported from the beet fields to the factory by trucks and it is discharged into storage areas, with a limited capacity. The residence time beet spends there is a very important variable, as the 
percentage of sucrose in the beet decreases with this time. Unlike the optimization model, in this case this factor has been modeled explicitly to predict the consequences that any operation strategy could have on such matter. Thus, the model of this stage is a DAE system, where the mass stocked in the storage zone $\left(m_{B S t}\right)$ and its solids concentration $\left(\right.$ Brix $\left._{S t}\right)$ were computed using mass balances (B.1) and (B.2). To obtain the brix of the flow that leaves the storage zone $\left(\right.$ Brix $\left._{B o}\right)$, we used an empirical equation that depends on the time the beet spends stocked (B.4).

$$
\begin{gathered}
\frac{d m_{B S t}(t)}{d t}=W_{B i}(t)-W_{B o}(t) \\
\frac{d B r i x_{B S t}(t)}{d t}=\frac{W_{B i}(t)\left(\operatorname{Brix}_{B i}(t)-\operatorname{Brix}_{B S t}(t)\right)}{m_{B S t}(t)} \\
T_{r}(t)=\frac{m_{B S t}(t)}{W_{B o}(t)} \\
\operatorname{Brix}_{B o}(t)=B_{B i x}(t) \cdot\left(1-f\left(T_{r}(t)\right)\right)
\end{gathered}
$$

The arrival of trucks, $W_{B i}$ was modeled with a pulse function with a random frequency to represent problems in beet-sugar crops (Acebes et al., 1999).

\section{Diffusion stage}

In this stage, the most important equipment is the diffuser, where many internal complex processes are carried out. It is the largest process unit of a sugar factory and, strictly, it should be modeled as a distributed parameter system, which requires the use of Partial Differential Equations (PDEs), which involves an important computational cost. A complete dynamic simulator of a DDS extractor was developed by (Merino and Acebes, 2003). Using this simulator, it was estimated that, for our purposes, the diffusion operation performed within the diffuser could be replaced by a delayed first order system, which depends on the beet-input flow and brix, without much loss of information.

With respect to the steam demand of this stage, it was assumed that it is mainly due to the action of some heat exchangers used to reheat part of the juice 
recirculated from the diffuser (Merino, 2008). Therefore, such heat exchangers, that use part of the steam provided by the evaporation, were modeled using first-principles equations to compute the steam demand with accuracy.

\section{Purification}

In this stage, impurities contained in the juice obtained from the diffusion are removed using chemical processes like liming or carbonation. Although such processes are not relevant for our operation, as they do not demand steam nor an important electricity power, their dynamics cannot be neglected, and they have been approximated using first order systems with delay. Again, the presence of the training simulator was key in order to obtain and parametrize such models.

The main steam consumption of this stage comes from heating the juice before being treated in the chemical operations mentioned before. This step is critical in order to optimize the chemical processes performance. As we did in the diffusion stage, such heat exchangers were modeled using first-principles equations, where the steam demand depends on the juice mass flow and the temperature desired. The controllers related to such system were also modeled in detail.

\section{Evaporation stage}

The objective of the evaporation stage is to remove water from the purification juice until the sugar concentration is the one selected by the operator. The water is removed in the form of steam which is used as main heat source for the other parts of the factory. Considering that the evaporation stage is the main steam consumer and producer of the factory, we modeled it using detailed firstprinciples equations of the process units (evaporators, heat exchangers, tanks, pumps, valves and tubes) and we included a complete control structure. The model was built from a library of model components developed in (Merino A. 2008) using the graphical capabilities of the modeling tool in the same fashion as the CHP plant.

The model counts with a tank to store the clear juice obtained from the purification stage, four heat exchangers to rise the temperature of the juice, and six evaporators to increase the sugar concentration. Besides, there are valves, pumps, and controllers to manage the level of the tank and the evaporators, the 
input and output steam pressure, and the sugar concentration of the output syrup. The steam economy of the factory falls on how the evaporation and the crystallization stages work. In particular, the steam demand of the crystallization mainly depends on the sugar concentration of the syrup produced in the evaporation and the pressure used to operate the crystallizers. So, the modeled control structure allows the consideration of the set-points of the mentioned controlled variables as input variables.

\section{Crystallization stage}

According to the description of the sugar production process made in section 4.1, the crystallization stage is a very complicated set of processes, where continuous and batch operations are performed in a sequential way to first crystallize, and later, separate the sugar from the syrup obtained in the evaporation stage. A detailed model of the process of sugar crystals formation, along with the separation stage based on a centrifugation process can be quite complicated. In (Mazaeda, 2010), both problems were studied, and an approach based on population balances was proposed to model the crystals formation yielding a model with an important computational cost.

According to the aims of the simulation module, and based on the crystallization model previously mentioned, we developed a simple model that describes the average steam demand from this stage as a function of the syrup mass flow, its sugar concentration, and the pressure used to operate the crystallizers:

$$
W_{S C r}(t)=K_{C r} \cdot W_{S y C r}(t) \cdot \frac{100-B_{\text {Brix }}}{100} \cdot \frac{H_{\text {Sat }}(1.5)}{H_{\text {Sat }}(\text { Boiling Pressure })}
$$

Where:

- $W_{S c r}$ : Average steam mass flow demanded by the crystallization stage.

- $W_{S y C r}$ : Syrup mass flow entering the crystallization stage.

- Brix $_{S y}$ : Brix (sugar concentration) of the input syrup.

- $H_{\text {Sat }}$ : Enthalpy of saturated steam at a determined pressure.

- $K_{C r}$ : Constant to be obtained by experimentation. 
Additionally, a first order dynamic was added to the previous calculated average value. It is important to emphasize that with this model we are assuming that the crystallizers are perfectly synchronized, and the steam is demanded without oscillations of any kind. This is not how the real process works, so, to make a more realistic approach, we added some negative and positive pulses to the average value, whose magnitude and frequency can be selected by the user.

\section{Power consumption}

So far, we have only described how we have modeled the heat energy consumption of each part of the beet-sugar factory, however, we are also interested in the electricity consumption of each section. A typical sugar factory is composed of many pieces of equipment which need power supply to work. However, modeling the individual power consumption of each component would require a degree of complexity that is not necessary for the intended purpose of this simulator. Thus, to reduce the complexity of the model, the power consumption was calculated as an average of each of the main stages of the plant, being the overall energy consumption the sum of the consumption of each stage. To parametrize the consumption of each section, first, we took as an approximation the data found in (Urbaniec, 1989), which relates the electricity demand of a typical beet-sugar factory with its processing capability, yielding the curve shown in Figure B-3.

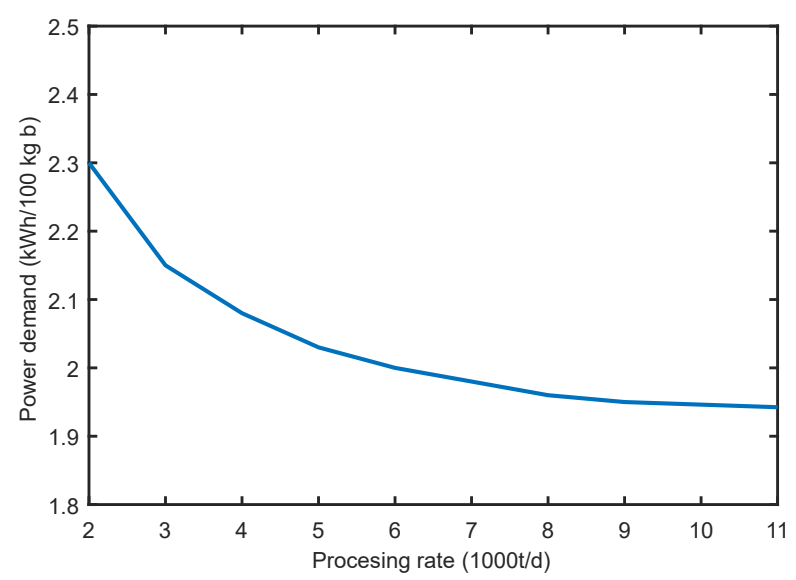

Figure B-3. Specific power consumption of a typical beet-sugar factory. 
As Figure B-3 shows, when the processing rate is increased, the specific power demand is reduced. The consumption of the main stages of the plant was estimated applying typical percentage values over the global consumption obtained from (Frankenfeld and Voss, 2004). Thus, it was possible to compute the power consumption of each stage depending on the processing rate, which is a natural way to incorporate the dynamic in the total electricity demand. Therefore, when the beet processing rate is increased, first we see that the power consumption of the diffusion increases, later the purification, and so on.

\section{Cogeneration plant}

In the cogeneration plant is where the steam and power demanded by the factory are produced. Due to its importance, the CHP plant has also been modeled using first-principles equations in a similar fashion to the evaporation stage (see Figure B-4). The modeled CHP plant mainly consists of boilers where steam is produced by boiling water using the energy obtained from the combustion of natural gas, and turbines where the steam is expanded to obtain electricity. As shown in Figure B-4, in our process, the cogeneration plant is composed of three boilers and three different backpressure steam turbines. Besides of being expanded in the turbines, the steam can also be bypassed directly to the evaporation section, and to protect the evaporation from high steam pressures, a relief valve has been modeled too.

The model used to represent the behavior of the boilers was described in (Pelayo, 1999). Apart from the steam generator, it considers the preheating of the feed water, the superheating of the steam obtained using heat exchangers fed by the combustion fumes, and a boilers typical control system. Regarding the turbines, the objective of this model is to simulate the steam expansion and the conversion of its thermal energy into electric power. Furthermore, it is necessary to simulate the connection with the external grid, being able to send and receive electricity, and the possibility of operating in island mode, i.e., disconnected from the grid and generating exactly the electricity demanded by the factory. This concept is key, given that depending on the operation mode, the controlled variable will be the generated power or the turbine axis rotational speed. Therefore, the turbine model obtained, which is based on (Chaibakhsh and Ghaffari, 2008; Thomas, 1999), represents both, the power generation, and the axis rotational speed. 


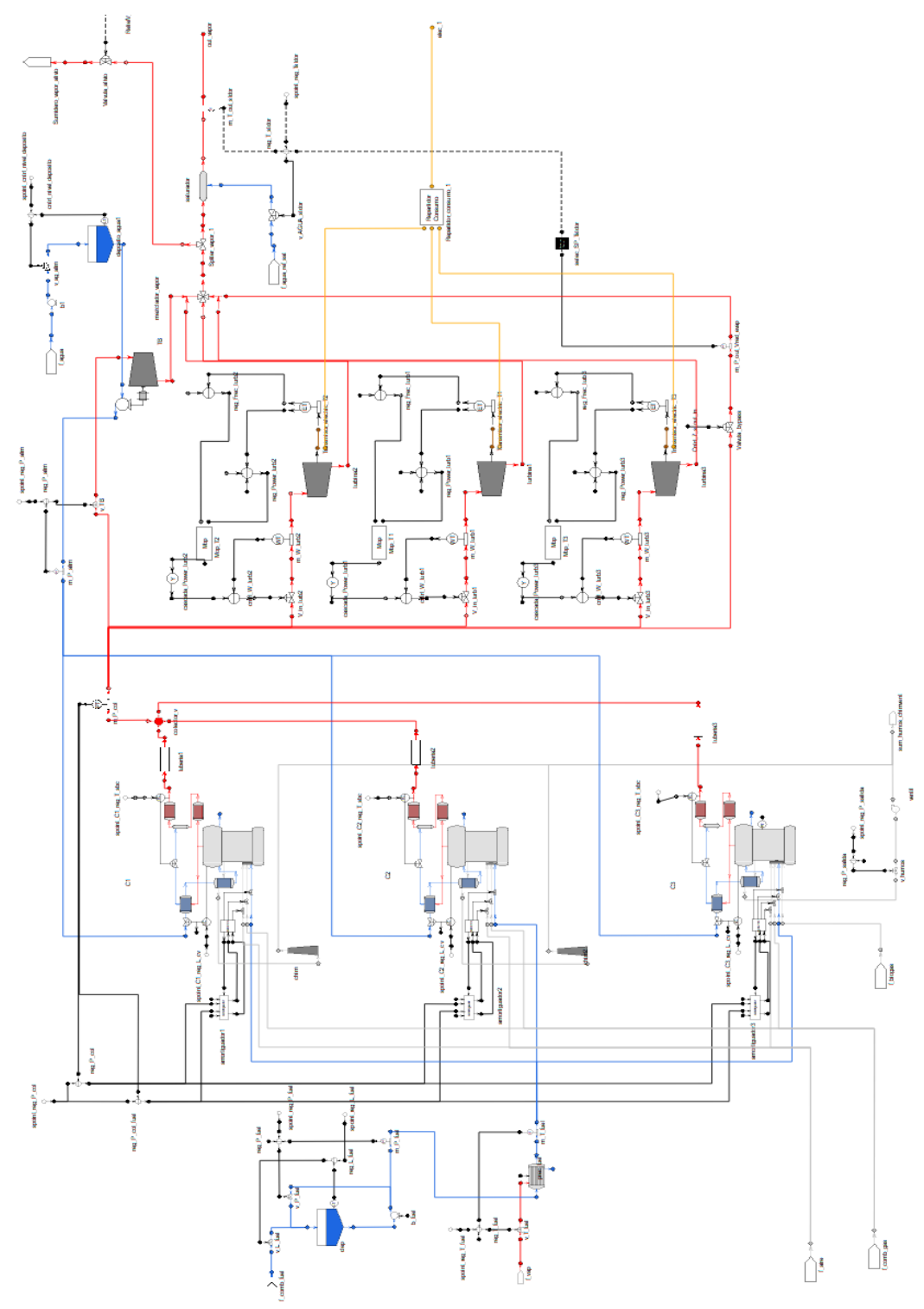

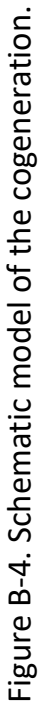




\section{Simulation size}

The order of magnitude of the simulation module can be estimated considering that it is composed of 6485 equations, being 449 of them dynamic. Furthermore, it counts with 2131 parameters and 29 boundary variables (see Table B-1). As model inputs, changes in the production specs and in the most important setpoints were considered. As outputs, besides the different operational variables related to the process, others associated with energy consumption were selected, like heat and power demand, cogeneration performance, fuel consumption, etc.

Table B-1. Summary of the simulation model specs.

\begin{tabular}{l|l}
\hline Number of equations & 6485 \\
\hline - Static & 6036 \\
- Dynamic & 449 \\
\hline Parameters & 2131 \\
\hline Variables & 6456 \\
\hline Inputs & 29 \\
\hline Non-linear algebraic loops & 7 \\
\hline
\end{tabular}

To measure the simulation speed, a test was performed where $48 \mathrm{~h}$ $(172,000 \mathrm{~s})$ of production were simulated, changing between different operational points. The real time used by the simulation was $274 \mathrm{~s}$, so the time ratio between simulation and real time is approximately 628 . 


\section{B.3 Visualization module}

The final aim of the visualization module in the presented architecture (Figure B1 ), is to give the final user a simple interface to interact with the simulation and the optimization module. To do so, we have used the SCADA software Wonderware ${ }^{\circledR}$ Intouch $^{\circledR}$, which is one of the most used in the process industry. The final HMI developed is presented in Figure B-5.

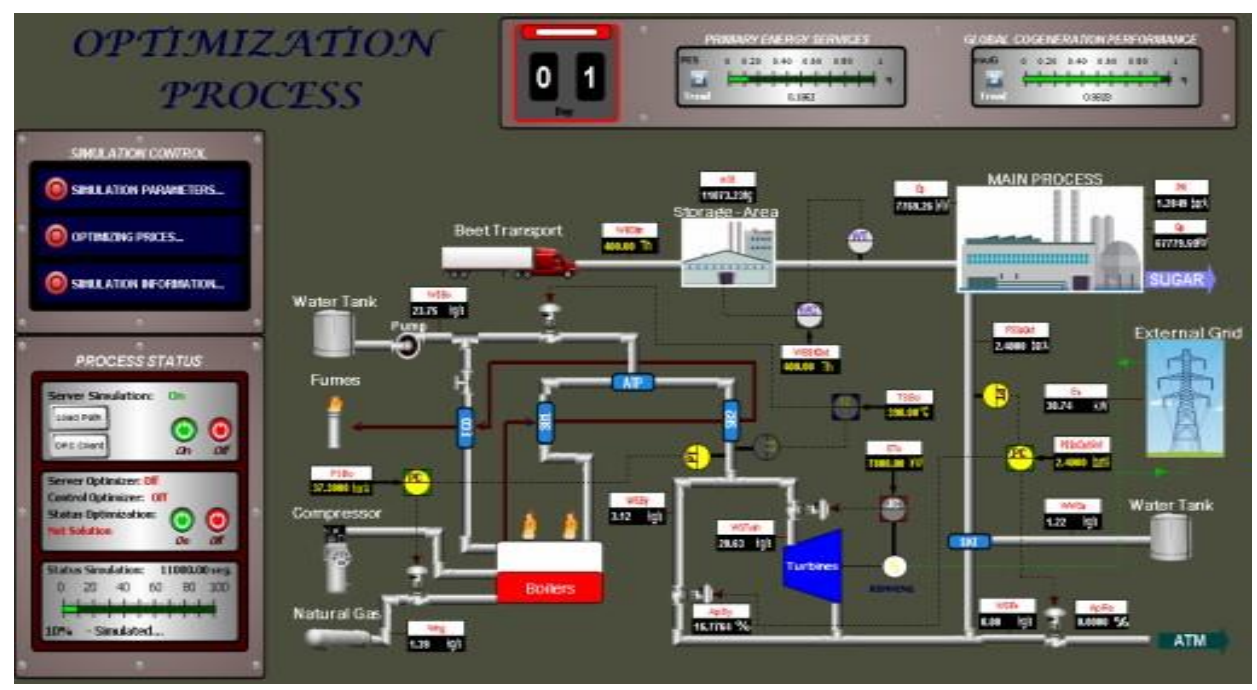

Figure B-5. Visualization module interface.

In the left side of Figure B-5, we find two different panels used to interact with the simulation and optimization modules. In the center, we see a big schematic where the most relevant variables of the system are displayed, giving a compact summary of the actual state of the plant to the end user. At the top, we show a counter that indicates the day that is being simulated, and two performance indexes: The Primary Energy Savings and the global efficiency of the process. We recall that we do not count with a real process in our architecture, however, in such case, this interface should be integrated with the rest of the SCADA, so the user could interact with the real process variables and test different operational strategies in simulation using the same software. This work was the product of an undergraduate thesis project, where the reader is referenced for more information (Sánchez, 2019). 


\section{B.4 Application example}

In this section, we follow the steps presented in Section 3.4 applied to our case study.

\section{1-Computation of the optimization initial condition.}

We recall that the first step consists in running a simulation from the actual state of the plant until midnight using the expected strategy until such time. To do so, in the lower panel, we find a module where the simulator path can be specified (see Figure B-6). In our case, the operation strategy is sent to the simulator through a file previously prepared with the expected inputs during time, so to run the simulation, we only have to press on the $O N$ button. We can also check the status of the connection pressing on the OPC client button.

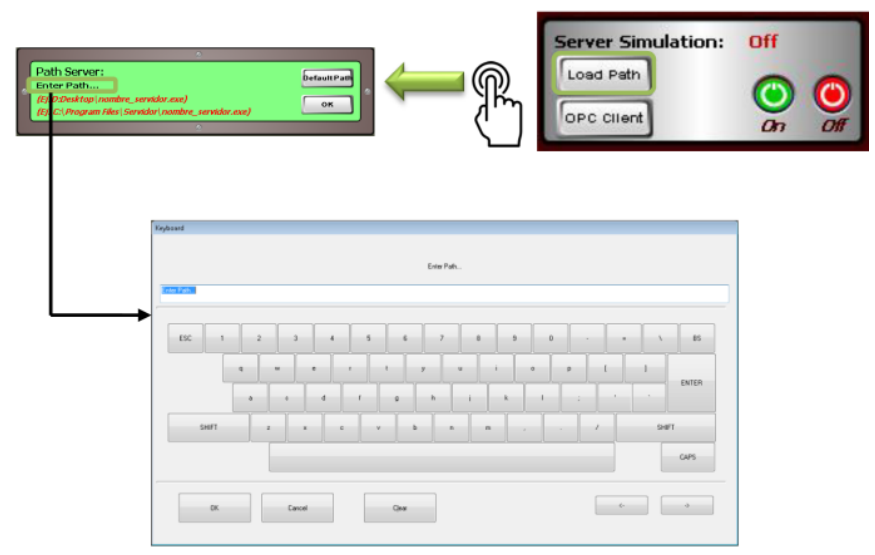

Figure B-6. Activating the simulation OPC UA server.

When the simulation is done, a new file with the simulation results is generated. In this stage we are only interested in the state of the process at the end of the simulation. Since Wonderware ${ }^{\circledR}$ Intouch ${ }^{\circledR}$ is a software prepared to work with real systems in real time, we have not been able to plot the simulation results in an XY graphic, so if the evolution of the plant up to that state would like to be consulted, it could be plotted using Python or other software like Matlab $^{\circledR}$. 


\section{2-Computation of the optimization results.}

With the initial condition computed in the previous step, before running an optimization, we need to specify the electricity price and the beet input expected for the next day. To do so, we must click on the upper panel in the option named Optimizing prices (see Figure B-7), and a new pop-up windows appears. Here, two different columns are visible where the user can manually set the desired values. In case that we only wanted to test operational strategies; we provide a data base with some interesting scenarios. To access to such data base, we only must click on the load data base button and select the desired scenario.

Since we found some incompatibilities when sending this information to the optimizer in a vector format, we have used Microsoft ${ }^{\circledR}$ Excel $^{\circledR}$ to send the exogeneous variables to the optimizer.
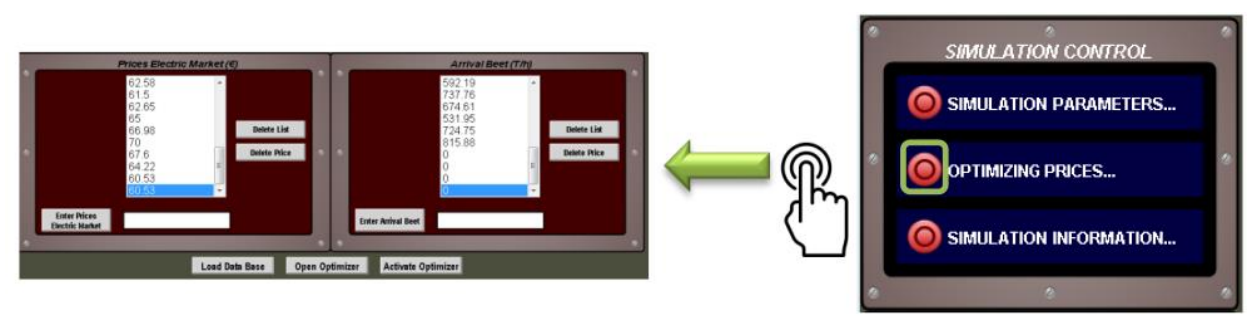

Figure B-7. Setting the expected electricity market price and beet arrival.

To activate the optimizer, first, we need to establish a connection with the optimization OPC UA server. To do so, we must press on the Open optimizer button. We can check the status of the connection looking at the optimization server panel shown in Figure B-8. During the optimization, the optimization status shown in Figure B-8, will change from not solution to calculating results, and finally, to solution found. The results are stored in a file ready to be plotted.

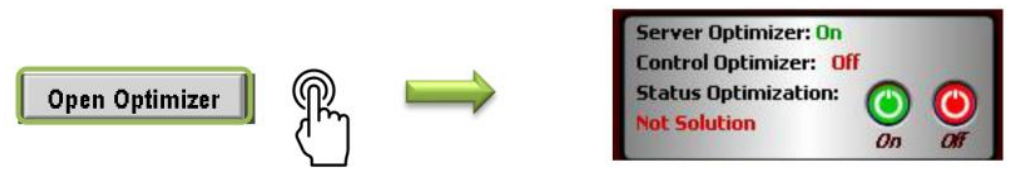

Figure B-8. Optimization server panel. 
Appendix B. Industrial roll-out concept applied to a beet-sugar process

\section{3-Optimization results evaluation}

The resulting file obtained in the previous step contains the optimal input and the prediction computed by the optimizer using its internal model. Considering that this model is much simpler than the one used in the simulation module, we can run a simulation using the optimization results and obtain a better approximation of the expected process response. An example of some of the variables that would see an operator is shown below.
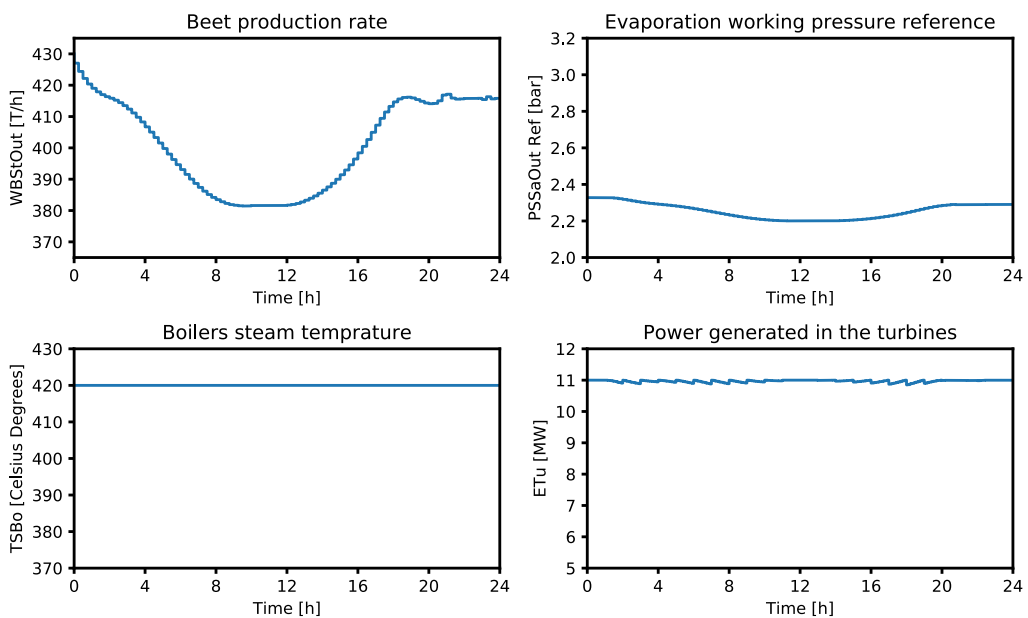

Figure B-9. Optimal inputs computed by the optimizer
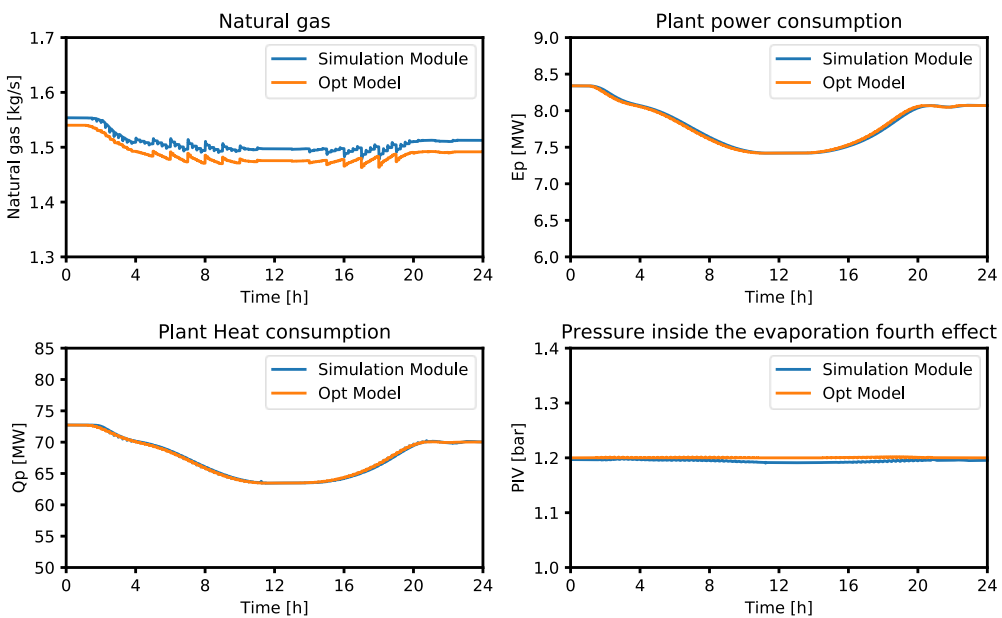

Figure B-10. Comparison between optimizer prediction and simulation module (1). 

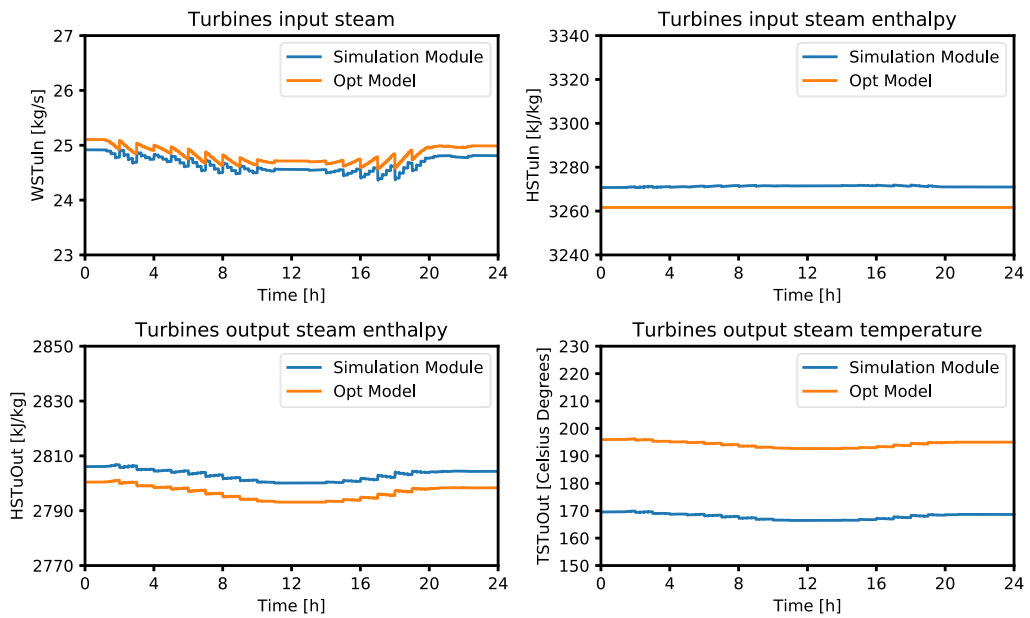

Figure B-11. Comparison between optimizer prediction and simulation module (2).
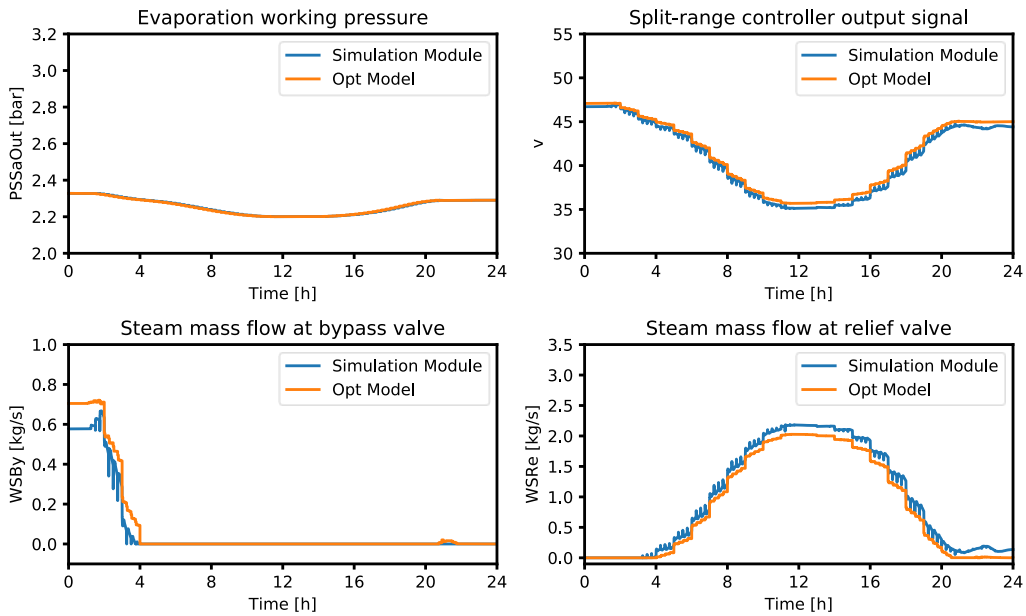

Figure B-12. Comparison between optimizer prediction and simulation module (3). 


\section{4-Results implementation}

After evaluating the optimization results, the user can decide to implement them manually, automatically, or neither and repeat the optimization with other inputs. To its automatic implementation, once we have found the solution, we have to press on the On button found in Figure B-8, which will change the control optimizer status from off to wait until midnight. In that moment the optimization results will be sent to the control layer, which, ideally, will be commanded by an MPC as discussed in Section 3.4. We recall that the user can stop the communication between the optimizer and the control system in any moment just pressing on the Off button. However, from that moment on, the user will have to give manually the inputs required.

Given that we are working with a simulated plant that is the same as the simulation module and we have not developed an MPC, the implemented results match exactly the ones shown in the figures shown in the previous section. 